REPRESENTATION THEORY

An Electronic Journal of the American Mathematical Society

Volume 14, Pages 446-509 (July 7, 2010)

S 1088-4165(2010)00379-9

\title{
TROPICAL R MAPS AND AFFINE GEOMETRIC CRYSTALS
}

\author{
MASAKI KASHIWARA, TOSHIKI NAKASHIMA, AND MASATO OKADO
}

\begin{abstract}
By modifying an earlier method of the authors (2008), certain affine geometric crystals are realized in affinization of the fundamental representation $W\left(\varpi_{1}\right)_{l}$, and the tropical $\mathrm{R}$ maps for the affine geometric crystals are described explicitly. We also define prehomogeneous geometric crystals and show that for a positive geometric crystal, the connectedness of the corresponding ultra-discretized crystal is the sufficient condition for prehomogeneity of the positive geometric crystal. Moreover, the uniqueness of tropical R maps is discussed.
\end{abstract}

\section{INTRODUCTION}

An R-matrix appears as a solution to the Yang-Baxter equation, which is a key to solve integrable lattice models in statistical mechanics. To understand Rmatrices representation theoretically, Drinfeld [D1 and Jimbo [J] introduced the quantized universal enveloping algebra. Once its representation theory has been established, an R-matrix is interpreted as an intertwiner between the tensor product of finite-dimensional modules and the one with the order of the tensor product being reversed. In [KMN1, KMN2 the notions of finite-dimensional modules and R-matrices acquired a combinatorial version by using the theory of crystal bases. In the papers, the objects, perfect crystals and combinatorial R-matrices, are introduced and play an important role to show that some physical quantities for particular vertex-type solvable models are equal to affine Lie algebra characters. Combinatorial R-matrices also play an essential role in some integrable cellular automata HKOTY.

The notion of geometric crystals for semi-simple algebraic groups has been initiated by Berenstein and Kazhdan BK], and it is extended for Kac-Moody groups in N1. Geometric crystals are constructed on some geometric objects, such as algebraic varieties, and has an analogous structure to crystals. The relation between crystals and geometric crystals is not only a simple analogy, but also a more direct functorial connection, called ultra-discretization/tropicalization. Indeed, in [N1, the geometric crystals on Schubert varieties of the corresponding Kac-Moody group are ultra-discretized to certain crystals (see 2.3). Applying this result, we constructed for an affine Lie algebra $\mathfrak{g}$ the affine geometric crystal $\mathcal{V}=\mathcal{V}(\mathfrak{g})$ in the fundamental $\mathfrak{g}$-representation $W\left(\varpi_{1}\right)$ in $\mathrm{KNO}$. Ultra-discretizing the geometric

Received by the editors September 2, 2008.

2010 Mathematics Subject Classification. Primary 17B37, 17B67; Secondary 22E65, 14 M15.

Key words and phrases. Prehomogeneous geometric crystal, perfect crystal, folding, tropical $R$ map, ultra-discretization.

This work was supported in part by JSPS Grants in Aid for Scientific Research, numbers 18340007(M.K.), 19540050(T.N.), 20540016(M.O.).

(C) 2010 American Mathematical Society Reverts to public domain 28 years from publication 
crystal $\mathcal{V}(\mathfrak{g})$, we obtained the limit of the coherent family of perfect crystals $B_{\infty}\left(\mathfrak{g}^{L}\right)$ where $\mathfrak{g}^{L}$ is the Langlands dual of $\mathfrak{g}$ (see [KKM]).

A tropical $\mathrm{R}$ map is an analogous object to the set-theoretic R ([D2]) and defined as follows (see 99): For a family of geometric crystals $X:=\left\{X_{\lambda}\right\}_{\lambda \in \Lambda}$ (parametrized by $\lambda \in \Lambda$ ) with a product structure, a birational map $R_{\lambda \mu}: X_{\lambda} \times X_{\mu} \rightarrow X_{\mu} \times X_{\lambda}$ $(\lambda, \mu \in \Lambda)$ is said to be a tropical $\mathrm{R}$ map, if it satisfies the Yang-Baxter equation and preserves the geometric crystal structure. In $[\mathrm{Y}]$ and $[\mathrm{KOTY}$, an explicit form of tropical R map is given for the geometric crystal $\mathcal{B}_{l}\left(l \in \mathbb{C}^{\times}\right)$of type $A_{n}^{(1)}$ and $D_{n}^{(1)}$. They obtained the tropical $\mathrm{R}$ map as a unique solution $\left(x^{\prime}, y^{\prime}\right)$ of the equation $M_{l}(x, z) M_{m}(y, z)=M_{m}\left(x^{\prime}, z\right) M_{l}\left(y^{\prime}, z\right)$ where $x \in \mathcal{B}_{l}, y \in \mathcal{B}_{m}, z$ is an indeterminate and $M_{l}(x, z)$ is a square matrix called an $M$-matrix (see 88 ).

In this paper, we construct the affine geometric crystal $\mathcal{V}_{l}$ in $W\left(\varpi_{1}\right)_{l}$, the affinization of the fundamental representation $W\left(\varpi_{1}\right)$, for $\mathfrak{g}=A_{n}^{(1)}, B_{n}^{(1)}, D_{n}^{(1)}$, $D_{n+1}^{(2)}, A_{2 n-1}^{(2)}$ and $A_{2 n}^{(2)}$. The geometric crystals $\mathcal{B}_{l}$ constructed in [KOTY are isomorphic to our geometric crystals $\mathcal{V}_{l}$. Thus, in these two cases we have the tropical $\mathrm{R}$ map for $\mathcal{V}_{l}$ through the isomorphism. By virtue of the "folding" method, we obtain the tropical R maps for the other cases; $B_{n}^{(1)}, D_{n+1}^{(2)}, A_{2 n-1}^{(2)}$ and $A_{2 n}^{(2)}$ from the tropical R map for $D_{N}^{(1)}$ with a suitable integer $N$. (The case $C_{n}^{(1)}$ cannot be obtained from folding $D_{N}^{(1)}$. This case will be discussed elsewhere.) Here note that the geometric crystal $\mathcal{V}(\mathfrak{g})$ is a special case of $\mathcal{V}(\mathfrak{g})_{l}$ for $l=1$.

Let us explain the construction of the tropical $\mathrm{R}$ map more precisely. First, we take a Dynkin diagram automorphism $\sigma$ for $D_{N}^{(1)}$, which induces the automorphism $\Sigma$ of the geometric crystal $\mathcal{B}_{l}$ of type $D_{N}^{(1)}$, where $\mathcal{B}_{l} \cong\left(\mathbb{C}^{\times}\right)^{2 N-2}$ as algebraic varieties. Let $X_{l}$ be the fixed-point variety for $\Sigma$, that is, $X_{l}:=\left\{x \in \mathcal{B}_{l} \mid \Sigma(x)=x\right\}$. There is an invertible matrix $J$ such that $M_{l}(\Sigma(x), z)=J M_{l}(x, z) J^{-1}\left(x \in \mathcal{B}_{l}\right)$. By this formula and the uniqueness of the solution for the equation $M_{l}(x, z) M_{m}(y, z)=$ $M_{m}\left(x^{\prime}, z\right) M_{l}\left(y^{\prime}, z\right)\left(l, m \in \mathbb{C}^{\times}\right)$, we deduce that the tropical $\mathrm{R}$ map sends $X_{l} \times$ $X_{m} \rightarrow X_{m} \times X_{l}$. Furthermore, this fixed-point variety $X_{l}$ is equipped with the $\mathfrak{g}^{\sigma}$-geometric crystal structure, where $\mathfrak{g}^{\sigma}$ is the affine Lie algebra obtained from $D_{N}^{(1)}$ by the folding associated with $\sigma$ and it is isomorphic to $\mathcal{V}_{l}$ for $\mathfrak{g}^{\sigma}$. Hence, we get the tropical R map for $\left\{\mathcal{V}_{l}\right\}$ in the case of $\mathfrak{g}^{\sigma}$.

In the article, we also discuss the uniqueness of the tropical $\mathrm{R}$ maps. As for usual R-matrices, it is unique (up to constant) by Schur's lemma, if the corresponding modules are irreducible. A similar situation occurs in the geometric crystals. A geometric crystal is prehomogeneous if there exists an open dense orbit by the actions of the $e_{i}^{c}$ 's. For $\phi, \phi^{\prime}$ two isomorphisms of prehomogeneous geometric crystals, if there exists a point $p$ in the open dense orbit which is sent to the same point by $\phi$ and $\phi^{\prime}$, it is shown that $\phi=\phi^{\prime}$ as rational maps. A crucial result in this article is that a positive geometric crystal $\mathbb{X}$ is prehomogeneous if the crystal ultradiscretized from $\mathbb{X}$ is connected (see Theorem [3.3). This uniqueness implies the following fact: For geometric crystals $X, Y, Z$, suppose that the product $X \times Y \times Z$ is prehomogeneous and that there exist tropical $\mathrm{R}$ maps for any two of $X, Y, Z$, then it follows from the uniqueness that we have the Yang-Baxter equation

$$
R^{(12)} R^{(23)} R^{(12)}=R^{(23)} R^{(12)} R^{(23)} .
$$

If $X \times Y$ is prehomogeneous, we also obtain the inversion formula

$$
R_{Y X} R_{X Y}=\mathrm{id}
$$


where $R^{(i j)}$ means $R$ acting on the $i$-th and the $j$-th components of the product. Namely, important properties of the tropical $R$ maps are deduced from the uniqueness. The tropical $\mathrm{R}$ map on the affine geometric crystal $\mathcal{V}_{l}$ introduced in this article is unique, since the ultra-discretized crystal $\mathcal{U} D\left(\mathcal{V}_{l}\right)$ and its tensor products are connected, which implies that $\mathcal{V}_{l}$ and its products are prehomogeneous.

\section{GeOmetric CRYstals AND CRYstals}

In this section, we review Kac-Moody groups and geometric crystals following $\mathrm{BK}$, $\mathrm{Kac}$, N1, N2, $\mathrm{PK}$.

2.1. Kac-Moody algebras and Kac-Moody groups. Fix a symmetrizable generalized Cartan matrix $A=\left(a_{i j}\right)_{i, j \in I}$ with a finite index set $I$. Let $\left(\mathfrak{t},\left\{\alpha_{i}\right\}_{i \in I}\right.$, $\left.\left\{\alpha_{i}^{\vee}\right\}_{i \in I}\right)$ be the associated root data, where $t$ is a vector space over $\mathbb{C}$ and $\left\{\alpha_{i}\right\}_{i \in I} \subset$ $\mathfrak{t}^{*}$ and $\left\{\alpha_{i}^{\vee}\right\}_{i \in I} \subset \mathfrak{t}$ are linearly independent satisfying $\alpha_{j}\left(\alpha_{i}^{\vee}\right)=a_{i j}$.

The Kac-Moody Lie algebra $\mathfrak{g}=\mathfrak{g}(A)$ associated with $A$ is the Lie algebra over $\mathbb{C}$ generated by $\mathfrak{t}$, the Chevalley generators $e_{i}$ and $f_{i}(i \in I)$ with the usual defining relations $\left([\mathrm{Kac})\right.$. There is the root space decomposition $\mathfrak{g}=\bigoplus_{\alpha \in \mathfrak{t}^{*}} \mathfrak{g}_{\alpha}$. Denote the set of roots by $\Delta:=\left\{\alpha \in \mathfrak{t}^{*} \mid \alpha \neq 0, \mathfrak{g}_{\alpha} \neq(0)\right\}$. Set $Q=\sum_{i} \mathbb{Z}_{i}, Q_{+}=\sum_{i} \mathbb{Z}_{\geq 0} \alpha_{i}$, $Q^{\vee}=\sum_{i} \mathbb{Z} \alpha_{i}^{\vee}$ and $\Delta_{+}=\Delta \cap Q_{+}$. An element of $\Delta_{+}$is called a positive root. Let $P \subset \mathfrak{t}^{*}$ be a weight lattice such that $\mathbb{C} \otimes P=\mathfrak{t}^{*}$ and $Q \subset P \subset\left\{\lambda \mid \lambda\left(Q^{\vee}\right) \subset \mathbb{Z}\right\}$, whose element is called a weight.

Define the simple reflections $s_{i} \in \operatorname{Aut}(\mathfrak{t})(i \in I)$ by $s_{i}(h):=h-\alpha_{i}(h) \alpha_{i}^{\vee}$, which generate the Weyl group $W$. It induces the action of $W$ on $\mathfrak{t}^{*}$ by $s_{i}(\lambda):=\lambda-\lambda\left(\alpha_{i}^{\vee}\right) \alpha_{i}$. Set $\Delta^{\text {re }}:=\left\{w\left(\alpha_{i}\right) \mid w \in W, i \in I\right\}$, whose element is called a real root.

Let $G$ be the Kac-Moody group associated with $(\mathfrak{g}, P)\left([\mathrm{PK})\right.$. Let $U_{\alpha}:=\exp \mathfrak{g}_{\alpha}$ ( $\alpha \in \Delta^{\mathrm{re}}$ ) be the one-parameter subgroup of $G$. The group $G$ is generated by $U_{\alpha}$ $\left(\alpha \in \Delta^{\text {re }}\right)$. Let $U^{ \pm}$be the subgroup generated by $U_{ \pm \alpha}\left(\alpha \in \Delta_{+}^{\text {re }}=\Delta^{\text {re }} \cap Q_{+}\right)$, i.e., $U^{ \pm}:=\left\langle U_{ \pm \alpha} \mid \alpha \in \Delta_{+}^{\mathrm{re}}\right\rangle$.

For any $i \in I$, there exists a unique group homomorphism $\phi_{i}: S L_{2}(\mathbb{C}) \rightarrow G$ such that

$$
\phi_{i}\left(\left(\begin{array}{ll}
1 & t \\
0 & 1
\end{array}\right)\right)=\exp \left(t e_{i}\right), \phi_{i}\left(\left(\begin{array}{ll}
1 & 0 \\
t & 1
\end{array}\right)\right)=\exp \left(t f_{i}\right) \quad(t \in \mathbb{C}) .
$$

Set $\alpha_{i}^{\vee}(c):=\phi_{i}\left(\left(\begin{array}{ll}c & 0 \\ 0 & c^{-1}\end{array}\right)\right), x_{i}(t):=\exp \left(t e_{i}\right), y_{i}(t):=\exp \left(t f_{i}\right), G_{i}:=\phi_{i}\left(S L_{2}(\mathbb{C})\right)$, $T_{i}:=\alpha_{i}^{\vee}\left(\mathbb{C}^{\times}\right)$and $N_{i}:=N_{G_{i}}\left(T_{i}\right)$. Let $T$ be the subgroup of $G$ with $P$ as its weight lattice which is called a maximal torus in $G$, and let $B^{ \pm}=U^{ \pm} T$ be the Borel subgroup of $G$. Let $N$ be the subgroup of $G$ generated by the $N_{i}$ 's. Then we have the isomorphism $\phi: W \stackrel{\sim}{\longrightarrow} N / T$ defined by $\phi\left(s_{i}\right)=N_{i} T / T$. An element $\bar{s}_{i}:=x_{i}(-1) y_{i}(1) x_{i}(-1)=\phi_{i}\left(\left(\begin{array}{cc}0 & -1 \\ 1 & 0\end{array}\right)\right)$ in $N_{G}(T)$ is a representative of $s_{i} \in W=$ $N_{G}(T) / T$.

2.2. Geometric crystals. Let $W$ be the Weyl group associated with $\mathfrak{g}$. Define $R(w)$ for $w \in W$ by

$$
R(w):=\left\{\left(i_{1}, i_{2}, \ldots, i_{l}\right) \in I^{l} \mid w=s_{i_{1}} s_{i_{2}} \cdots s_{i_{l}}\right\}
$$

where $l$ is the length of $w$, i.e., $R(w)$ is the set of reduced expressions of $w$.

Let $X$ be a variety, $\gamma_{i}: X \rightarrow \mathbb{C}$ and $\varepsilon_{i}: X \longrightarrow \mathbb{C}(i \in I)$ rational functions on $X$, and $e_{i}: \mathbb{C}^{\times} \times X \longrightarrow X\left((c, x) \mapsto e_{i}^{c}(x)\right)$ a rational $\mathbb{C}^{\times}$-action. For $w \in W$ and 
$\mathbf{i}=\left(i_{1}, \ldots, i_{l}\right) \in R(w)$, set $\alpha^{(j)}:=s_{i_{l}} \cdots s_{i_{j+1}}\left(\alpha_{i_{j}}\right)(1 \leq j \leq l)$ and

$$
\begin{aligned}
e_{\mathbf{i}}: T \times X & \rightarrow X \\
(t, x) & \mapsto e_{\mathbf{i}}^{t}(x):=e_{i_{1}}^{\alpha^{(1)}(t)} e_{i_{2}}^{\alpha^{(2)}(t)} \cdots e_{i_{l}}^{\alpha^{(l)}(t)}(x) .
\end{aligned}
$$

Definition 2.1. A quadruple $\left(X,\left\{e_{i}\right\}_{i \in I},\left\{\gamma_{i},\right\}_{i \in I},\left\{\varepsilon_{i}\right\}_{i \in I}\right)$ is a $G$ (or $\mathfrak{g}$ )-geometric crystal if:

(i) $\{1\} \times X \cap \operatorname{dom}\left(e_{i}\right)$ is open dense in $\{1\} \times X$ for any $i \in I$. Here $\operatorname{dom}\left(e_{i}\right)$ is the domain of definition of $e_{i}: \mathbb{C}^{\times} \times X \rightarrow X$.

(ii) $\gamma_{j}\left(e_{i}^{c}(x)\right)=c^{a_{i j}} \gamma_{j}(x)$.

(iii) $e_{\mathbf{i}}=e_{\mathbf{i}^{\prime}}$ for any $w \in W$ and $\mathbf{i}$. $\mathbf{i}^{\prime} \in R(w)$.

(iv) $\varepsilon_{i}\left(e_{i}^{c}(x)\right)=c^{-1} \varepsilon_{i}(x)$.

Note that condition (iii) is equivalent to the following so-called Verma relations:

$$
\begin{array}{ll}
e_{i}^{c_{1}} e_{j}^{c_{2}}=e_{j}^{c_{2}} e_{i}^{c_{1}} & \text { if } a_{i j}=a_{j i}=0, \\
e_{i}^{c_{1}} e_{j}^{c_{1} c_{2}} e_{i}^{c_{2}}=e_{j}^{c_{2}} e_{i}^{c_{1} c_{2}} e_{j}^{c_{1}} & \text { if } a_{i j}=a_{j i}=-1, \\
e_{i}^{c_{1}} e_{j}^{c_{1}^{2} c_{2}} e_{i}^{c_{1} c_{2}} e_{j}^{c_{2}}=e_{j}^{c_{2}} e_{i}^{c_{1} c_{2}} e_{j}^{c_{1}^{2} c_{2}} e_{i}^{c_{1}} & \text { if } a_{i j}=-2, a_{j i}=-1, \\
e_{i}^{c_{1}} e_{j}^{c_{1}^{3} c_{2}} e_{i}^{c_{1}^{2} c_{2}} e_{j}^{c_{1}^{3} c_{2}^{2}} e_{i}^{c_{1} c_{2}} e_{j}^{c_{2}}=e_{j}^{c_{2}} e_{i}^{c_{1} c_{2}} e_{j}^{c_{1}^{3} c_{2}^{2}} e_{i}^{c_{1}^{2} c_{2}} e_{j}^{c_{1}^{3} c_{2}} e_{i}^{c_{1}} & \text { if } a_{i j}=-3, a_{j i}=-1,
\end{array}
$$

Note that the last formula is different from the one in BK, N1, N2 which seems to be incorrect. If $\mathbb{X}=\left(X,\left\{e_{i}\right\},\left\{\gamma_{i}\right\},\left\{\varepsilon_{i}\right\}\right)$ satisfies conditions (i), (ii) and (iv), we call $\mathbb{X}$ a pregeometric crystal.

2.3. Geometric crystal on a Schubert cell. Let $X:=G / B$ be the flag variety, which is the inductive limit of finite-dimensional projective varieties. For $w \in W$, let $X_{w}:=B w B / B \subset X$ be the Schubert cell associated with $w$, which has a natural geometric crystal structure $([\mathrm{BK}],[\mathrm{N1}])$. For $\mathbf{i}=\left(i_{1}, \ldots, i_{k}\right) \in R(w)$, set

$$
B_{\mathbf{i}}^{-}:=\left\{Y_{\mathbf{i}}\left(c_{1}, \ldots, c_{k}\right):=Y_{i_{1}}\left(c_{1}\right) \cdots Y_{i_{l}}\left(c_{k}\right) \mid c_{1} \cdots, c_{k} \in \mathbb{C}^{\times}\right\} \subset B^{-}
$$

where $Y_{i}(c):=y_{i}\left(c^{-1}\right) \alpha_{i}^{\vee}(c)=\alpha_{i}^{\vee}(c) y_{i}(c)$. Then $B_{\mathbf{i}}^{-}$is birationally isomorphic to $X_{w}$ and endowed with the induced geometric crystal structure. Let $\xi$ be an element in the torus $T$. Then we can define the geometric crystal structure on $B_{\mathbf{i}}^{-} \cdot \xi$ and we shall describe its explicit form: The action $e_{i}^{c}$ on $B_{\mathbf{i}}^{-} \cdot \xi$ is given by

$$
e_{i}^{c}\left(Y_{i_{1}}\left(c_{1}\right) \cdots Y_{i_{l}}\left(c_{k}\right) \xi\right)=Y_{i_{1}}\left(\mathcal{C}_{1}\right) \cdots Y_{i_{l}}\left(\mathcal{C}_{k}\right) \xi
$$

where

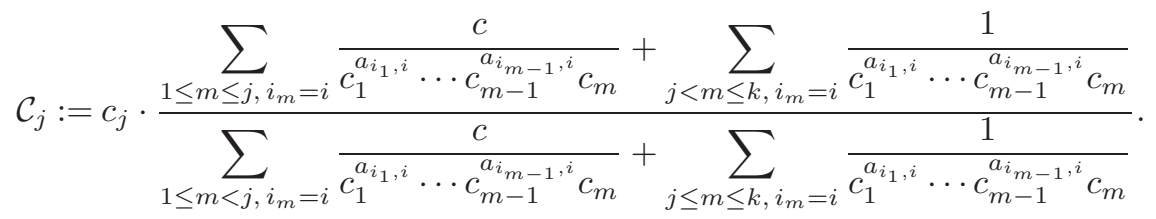

The explicit forms of rational functions $\varepsilon_{i}$ and $\gamma_{i}$ are:

$$
\begin{gathered}
\varepsilon_{i}\left(Y_{i_{1}}\left(c_{1}\right) \cdots Y_{i_{l}}\left(c_{k}\right) \xi\right)=\sum_{1 \leq m \leq k, i_{m}=i} \frac{1}{c_{1}^{a_{i_{1}, i}} \cdots c_{m-1}^{a_{i_{m}-1, i}} c_{m}}, \\
\gamma_{i}\left(Y_{i_{1}}\left(c_{1}\right) \cdots Y_{i_{l}}\left(c_{k}\right) \xi\right)=c_{1}^{a_{i_{1}, i}} \cdots c_{k}^{a_{i_{k}, i}} \alpha_{i}(\xi) .
\end{gathered}
$$

These will be needed in Section 5 to construct affine geometric crystals. 
2.4. Crystals. We recall the notion of crystals, which is obtained by abstracting the combinatorial properties of crystal bases.

Definition 2.2. A crystal $B$ is a set endowed with the following maps:

$$
\begin{aligned}
& \text { wt: } B \longrightarrow P, \\
& \varepsilon_{i}: B \longrightarrow \mathbb{Z} \sqcup\{-\infty\}, \quad \varphi_{i}: B \longrightarrow \mathbb{Z} \sqcup\{-\infty\} \quad \text { for } i \in I, \\
& \tilde{e}_{i}: B \sqcup\{0\} \longrightarrow B \sqcup\{0\}, \quad \tilde{f}_{i}: B \sqcup\{0\} \longrightarrow B \sqcup\{0\} \quad \text { for } i \in I, \\
& \tilde{e}_{i}(0)=\tilde{f}_{i}(0)=0 .
\end{aligned}
$$

Those maps satisfy the following axioms: For all $b, b_{1}, b_{2} \in B$, we have

$$
\begin{aligned}
& \varphi_{i}(b)=\varepsilon_{i}(b)+\left\langle\alpha_{i}^{\vee}, \operatorname{wt}(b)\right\rangle, \\
& \operatorname{wt}\left(\tilde{e}_{i} b\right)=\operatorname{wt}(b)+\alpha_{i} \text { if } \tilde{e}_{i} b \in B, \\
& \operatorname{wt}\left(\tilde{f}_{i} b\right)=\operatorname{wt}(b)-\alpha_{i} \text { if } \tilde{f}_{i} b \in B, \\
& \tilde{e}_{i} b_{2}=b_{1} \Longleftrightarrow \tilde{f}_{i} b_{1}=b_{2}, \\
& \varepsilon_{i}(b)=-\infty \Longrightarrow \tilde{e}_{i} b=\tilde{f}_{i} b=0 .
\end{aligned}
$$

Example 2.3. (i) If $(L, B)$ is a crystal base, then $B$ is a crystal.

(ii) For the crystal base $(L(\infty), B(\infty))$ of the subalgebra $U_{q}^{-}(\mathfrak{g})$ of the quantum algebra $U_{q}(\mathfrak{g}), B(\infty)$ is a crystal.

(iii) For $\lambda \in P$, set $T_{\lambda}:=\left\{t_{\lambda}\right\}$. We define a crystal structure on $T_{\lambda}$ by

$$
\tilde{e}_{i}\left(t_{\lambda}\right)=\tilde{f}_{i}\left(t_{\lambda}\right)=0, \quad \varepsilon_{i}\left(t_{\lambda}\right)=\varphi_{i}\left(t_{\lambda}\right)=-\infty, \quad \operatorname{wt}\left(t_{\lambda}\right)=\lambda .
$$

Definition 2.4. (i) To a crystal $B$, a colored oriented graph is associated by

$$
b_{1} \stackrel{i}{\longrightarrow} b_{2} \Longleftrightarrow \tilde{f}_{i} b_{1}=b_{2} .
$$

We call this graph the crystal graph of $B$.

(ii) A crystal $B$ is said to be connected, if its crystal graph is connected as a graph.

(iii) A crystal $B$ is free if $\tilde{e}_{i}^{n}(b) \neq 0$ and $\tilde{f}_{i}^{n}(b) \neq 0$ for any $b \in B, i \in I$ and $n>0$.

2.5. Positive structure, ultra-discretization and tropicalization. Let us recall the notions of positive structure and ultra-discretization/tropicalization.

Set $R:=\mathbb{C}(c)$ and define

$$
\begin{array}{cccc}
v: \quad R \backslash\{0\} & \longrightarrow & \mathbb{Z} \\
& f(c) & \mapsto & \operatorname{deg}(f(c)) .
\end{array}
$$

Here deg is the degree of poles at $c=\infty$. Note that for $f_{1}, f_{2} \in R \backslash\{0\}$, we have

$$
v\left(f_{1} f_{2}\right)=v\left(f_{1}\right)+v\left(f_{2}\right), \quad v\left(\frac{f_{1}}{f_{2}}\right)=v\left(f_{1}\right)-v\left(f_{2}\right) .
$$

We say that a non-zero rational function $f(c) \in \mathbb{C}(c)$ is positive if $f$ can be expressed as a ratio of polynomials with positive coefficients. Note that $f \in \mathbb{C}(c)$ is positive if and only if any pole of $f$ is not a positive number and $f(x)>0$ for any $x>0$.

If $f_{1}, f_{2} \in R$ are positive, then we have

$$
v\left(f_{1}+f_{2}\right)=\max \left(v\left(f_{1}\right), v\left(f_{2}\right)\right) .
$$

Let $T \simeq\left(\mathbb{C}^{\times}\right)^{l}$ be an algebraic torus over $\mathbb{C}$ and $X^{*}(T):=\operatorname{Hom}\left(T, \mathbb{C}^{\times}\right)$(resp. $\left.X_{*}(T):=\operatorname{Hom}\left(\mathbb{C}^{\times}, T\right)\right)$ the lattice of characters (resp. co-characters) of $T$. We 
denote by $T_{+}$the set of points $x$ in $T$ such that $\chi(x)>0$ for any character $\chi$. Then $\left(\left(\mathbb{C}^{\times}\right)^{n}\right)_{+}=\left(\mathbb{R}_{>0}\right)^{n}$.

A non-zero rational function on an algebraic torus $T$ is called positive if it is written as $g / h$ where $g$ and $h$ are a positive linear combination of characters of $T$.

Definition 2.5. Let $f: T \rightarrow T^{\prime}$ be a rational mapping between two algebraic tori $T$ and $T^{\prime}$. We say that $f$ is positive, if $\mathbb{X} \circ f$ is positive for any character $\mathbb{X}: T^{\prime} \rightarrow \mathbb{C}$.

Denote by $\operatorname{Mor}^{+}\left(T, T^{\prime}\right)$ the set of positive rational mappings from $T$ to $T^{\prime}$.

Note that any $f \in \operatorname{Mor}^{+}\left(T, T^{\prime}\right)$ induces a real analytic map $f_{+}: T_{+} \rightarrow T_{+}^{\prime}$.

Lemma 2.6 ( $[\mathrm{BK}])$. For any $f \in \operatorname{Mor}^{+}\left(T_{1}, T_{2}\right)$ and $g \in \operatorname{Mor}^{+}\left(T_{2}, T_{3}\right)$, the composition $g \circ f$ is well defined and belongs to $\operatorname{Mor}^{+}\left(T_{1}, T_{3}\right)$.

By Lemma 2.6 we can define a category $\mathcal{T}_{+}$whose objects are algebraic tori over $\mathbb{C}$ and arrows are positive rational mappings. The category $\mathcal{T}_{+}$admits products. For two algebraic tori $T$ and $T^{\prime}$, their product in $\mathcal{T}_{+}$coincides with the usual product of $T$ and $T^{\prime}$.

Note that $T \mapsto T_{+}$gives a functor from $\mathcal{T}_{+}$to the category of real analytic manifolds.

Let $f: T \rightarrow T^{\prime}$ be a positive rational mapping of algebraic tori $T$ and $T^{\prime}$. We define a map $\widehat{f}: X_{*}(T) \rightarrow X_{*}\left(T^{\prime}\right)$ by

$$
\langle\chi, \widehat{f}(\xi)\rangle=v(\chi \circ f \circ \xi),
$$

where $\chi \in X^{*}\left(T^{\prime}\right)$ and $\xi \in X_{*}(T)$. Note that $\chi \circ f \circ \xi$ is a rational map from $\mathbb{C}^{\times}$ to itself.

Lemma $2.7([\mathrm{BK}])$. For any algebraic tori $T_{1}, T_{2}, T_{3}$, and positive rational mappings $f \in \operatorname{Mor}^{+}\left(T_{1}, T_{2}\right), g \in \operatorname{Mor}^{+}\left(T_{2}, T_{3}\right)$, we have $\widehat{g \circ f}=\widehat{g} \circ \widehat{f}$.

By this lemma, we obtain a functor

$$
\begin{array}{cccc}
\mathcal{U} D: & \mathcal{T}_{+} & & \text {Set } \\
T & & & X_{*}(T) \\
\left(f: T \rightarrow T^{\prime}\right) & & & \left(\widehat{f}: X_{*}(T) \rightarrow X_{*}\left(T^{\prime}\right)\right) .
\end{array}
$$

Let us come back to the situation in $\$ 2.2$ Hence $G$ is a Kac-Moody group and $T$ is its Cartan subgroup.

Definition 2.8 ( $\mathrm{BK}])$. Let $\mathbb{X}=\left(X,\left\{e_{i}\right\}_{i \in I},\left\{\gamma_{i}\right\}_{i \in I},\left\{\varepsilon_{i}\right\}_{i \in I}\right)$ be a $G$ (or g)geometric crystal, $T^{\prime}$ an algebraic torus and $\theta: T^{\prime} \rightarrow X$ a birational mapping. The mapping $\theta$ is called a positive structure on $\mathbb{X}$ if it satisfies:

(i) for any $i \in I$ the rational functions $\gamma_{i} \circ \theta: T^{\prime} \rightarrow \mathbb{C}$ and $\varepsilon_{i} \circ \theta: T^{\prime} \rightarrow \mathbb{C}$ are positive,

(ii) for any $i \in I$, the rational mapping $e_{i, \theta}: \mathbb{C}^{\times} \times T^{\prime} \rightarrow T^{\prime}$ defined by $e_{i, \theta}(c, t):=\theta^{-1} \circ e_{i}^{c} \circ \theta(t)$ is positive.

Let $\theta: T^{\prime} \rightarrow X$ be a positive structure on a geometric crystal $\mathbb{X}=\left(X,\left\{e_{i}\right\}_{i \in I}\right.$, $\left.\left\{\gamma_{i}\right\}_{i \in I},\left\{\varepsilon_{i}\right\}_{i \in I}\right)$. Applying the functor $\mathcal{U} D$ to the positive rational mappings $e_{i, \theta}: \mathbb{C}^{\times} \times T^{\prime} \rightarrow T^{\prime}$ and $\gamma_{i} \circ \theta, \varepsilon_{i} \circ \theta: T^{\prime} \rightarrow \mathbb{C}^{\times}$, we obtain

$$
\begin{aligned}
& \tilde{e}_{i}:=\mathcal{U} D\left(e_{i, \theta}\right): \mathbb{Z} \times X_{*}\left(T^{\prime}\right) \rightarrow X_{*}\left(T^{\prime}\right), \\
& \mathrm{wt}_{i}:=\mathcal{U} D\left(\gamma_{i} \circ \theta\right), \varepsilon_{i}:=\mathcal{U} D\left(\varepsilon_{i} \circ \theta\right): X_{*}\left(T^{\prime}\right) \rightarrow \mathbb{Z} .
\end{aligned}
$$


Hence the quadruple $\left(X_{*}\left(T^{\prime}\right),\left\{\tilde{e}_{i}\right\}_{i \in I},\left\{\mathrm{wt}_{i}\right\}_{i \in I},\left\{\varepsilon_{i}\right\}_{i \in I}\right)$ is a free pre-crystal structure (see [BK, 2.2]) and we denote it by $\mathcal{U} D_{\theta}(\mathbb{X})=\mathcal{U} D_{\theta, T^{\prime}}(\mathbb{X})$. As for the definition of crystal, see 4.11 or [KKM], K1], K2. We thus have the following theorem:

Theorem 2.9 ([BK], [N1]). For any geometric crystal $\mathbb{X}=\left(X,\left\{e_{i}\right\}_{i \in I},\left\{\gamma_{i}\right\}_{i \in I}\right.$, $\left.\left\{\varepsilon_{i}\right\}_{i \in I}\right)$ and a positive structure $\theta: T^{\prime} \rightarrow X$, the associated precrystal $\mathcal{U} D_{\theta, T^{\prime}}(\mathbb{X})$ is a crystal (see [BK, 2.2]).

Now, let $\mathcal{G} C^{+}(\mathfrak{g})$ be the category whose object is a triplet $\left(\mathbb{X}, T^{\prime}, \theta\right)$ where $\mathbb{X}=$ $\left(X,\left\{e_{i}\right\},\left\{\gamma_{i}\right\},\left\{\varepsilon_{i}\right\}\right)$ is a $\mathfrak{g}$-geometric crystal and $\theta: T^{\prime} \rightarrow X$ is a positive structure on $\mathbb{X}$, and a morphism $f:\left(\mathbb{X}_{1}, T_{1}^{\prime}, \theta_{1}\right) \rightarrow\left(\mathbb{X}_{2}, T_{2}^{\prime}, \theta_{2}\right)$ is given by a morphism $\varphi: X_{1} \longrightarrow$ $X_{2}$ of geometric crystals such that

$$
f:=\theta_{2}^{-1} \circ \varphi \circ \theta_{1}: T_{1}^{\prime} \longrightarrow T_{2}^{\prime},
$$

is a positive rational mapping. Let $\mathcal{C} R(\mathfrak{g})$ be the category of $\mathfrak{g}$-crystals. Then by the theorem above, we have

Corollary 2.10. $\mathcal{U} D$ defines a functor

$$
\begin{gathered}
\mathcal{U} D: \mathcal{G} C^{+}(\mathfrak{g}) \longrightarrow \mathcal{C} R\left(\mathfrak{g}^{L}\right), \\
\left(\mathbb{X}, T^{\prime}, \theta\right) \mapsto X_{*}\left(T^{\prime}\right), \\
\left(f:\left(\mathbb{X}_{1}, T_{1}^{\prime}, \theta_{1}\right) \rightarrow\left(\mathbb{X}_{2}, T_{2}^{\prime}, \theta_{2}\right)\right) \mapsto\left(\widehat{f}: X_{*}\left(T_{1}^{\prime}\right) \rightarrow X_{*}\left(T_{2}^{\prime}\right)\right),
\end{gathered}
$$

where $\mathfrak{g}^{L}$ is the Langlands dual for $\mathfrak{g}$.

We call the functor $\mathcal{U} D$ an "ultra-discretization" as in [N1], [N2]. While for a crystal $B \in \mathcal{C} R(\mathfrak{g})$, if there exists an object $\left(\mathbb{X}, T^{\prime}, \theta\right)$ in $\mathcal{G} C^{+}\left(\mathfrak{g}^{L}\right)$, we call $(\mathbb{X}, \theta)$ a tropicalization of $B$.

\section{Prehomogeneous geometric crystals}

Definition 3.1. Let $\mathbb{X}=\left(X,\left\{e_{i}^{c}\right\},\left\{\gamma_{i}\right\},\left\{\varepsilon_{i}\right\}\right)$ be a geometric crystal. We say that $\mathbb{X}$ is prehomogeneous if there exists a Zariski open dense subset $\Omega \subset X$ which is an orbit by the actions of the $e_{i}^{c}$ 's.

The following lemma is obvious.

Lemma 3.2. Let $\mathbb{X}_{j}=\left(X_{j},\left\{e_{i}^{c}\right\},\left\{\gamma_{i}\right\},\left\{\varepsilon_{i}\right\}\right)(j=1,2)$ be geometric crystals and $\mathbb{X}_{1}$ prehomogeneous. Let $\Omega_{1} \subset X_{1}$ be an open dense orbit in $X_{1}$. For isomorphisms of geometric crystals $\phi, \phi^{\prime}: \mathbb{X}_{1} \rightarrow \mathbb{X}_{2}$, suppose that there exists $p_{1} \in \Omega_{1}$ such that $\phi\left(p_{1}\right)=\phi^{\prime}\left(p_{1}\right) \in X_{2}$. Then, we have $\phi=\phi^{\prime}$ as rational maps.

The following is the criterion for the prehomogeneity of a geometric crystal.

Theorem 3.3. Let $\mathbb{X}=\left(X,\left\{e_{i}^{c}\right\},\left\{\gamma_{i}\right\},\left\{\varepsilon_{i}\right\}\right)$ be a finite-dimensional positive geometric crystal with the positive structure $\theta: T \rightarrow X$ and $B:=\mathcal{U} D_{\theta}(\mathbb{X})$ the crystal obtained as the ultra-discretization of $\mathbb{X}$. If $B$ is a connected crystal, then $\mathbb{X}$ is prehomogeneous.

Proof. Set $m:=\operatorname{dim} T=\operatorname{dim} X$. We identify $T$ and $X$ by $\theta$, and take $\mathbf{a} \in T_{+}$(see 2.5). Assume that $B$ is connected and $\mathbb{X}(=T)$ is not prehomogeneous. Then there exists a nowhere dense closed subset $Z$ of $T$ such that $Z$ contains a and is invariant by the actions of the $e_{i}^{c}$ 's. 
Suppose that $Z$ is contained in a variety $\{x \in T \mid \phi(x)=0\}$ where

$$
\phi(x)=\sum_{\alpha \in \mathbb{Z}_{\geq 0}^{m}} a_{\alpha} x_{1}^{\alpha_{1}} \cdots x_{m}^{\alpha_{m}} \in \mathbb{C}\left[x_{1}, \ldots, x_{m}\right]
$$

is a non-zero polynomial. Set $A:=\left\{\alpha=\left(\alpha_{1}, \ldots, \alpha_{m}\right) \in \mathbb{Z}_{>0}^{m} \mid a_{\alpha} \neq 0\right\} \neq \emptyset$ and let $H$ be the convex hull of $A$ in $\mathbb{Z}^{m}$. Let us take an end point $\tilde{\alpha}$ of $H$. Then $\tilde{\alpha} \in A$ and there exists $c=\left(c_{1}, \cdots, c_{m}\right) \in B=\mathbb{Z}^{m}$ such that $\sum_{i} \tilde{\alpha}_{i} c_{i}>\sum_{i} \alpha_{i} c_{i}$ for any $\alpha \in A \backslash\{\tilde{\alpha}\}$. Since the crystal $B$ is connected, there exist $i_{1}, \ldots, i_{l} \in I$ and $k_{1}, \ldots, k_{l} \in \mathbb{Z}$ such that

$$
c=\left(c_{1}, \ldots, c_{m}\right)=\tilde{e}_{i_{1}}^{k_{1}} \cdots \tilde{e}_{i_{l}}^{k_{l}}(0,0, \ldots, 0) .
$$

We define rational functions $f_{1}(t), \ldots, f_{m}(t)$ by

$$
\left(f_{1}(t), \ldots, f_{m}(t)\right):=e_{i_{1}}^{t^{k_{1}}} \cdots e_{i_{l}}^{t^{k_{l}}} \mathbf{a} \in Z .
$$

Since $\tilde{e}_{i}=\mathcal{U} D\left(e_{i, \theta}\right)$, we have $v\left(f_{i}(t)\right)=c_{i}$, where $v: \mathbb{C}(t) \backslash\{0\} \rightarrow \mathbb{Z}$ is as in 2.5 . Thus we have

$$
v\left(\phi\left(f_{1}(t), \cdots, f_{m}(t)\right)\right)=\sum_{i} \tilde{\alpha}_{i} c_{i}
$$

which implies $\phi\left(f_{1}(t), \cdots, f_{m}(t)\right) \neq 0$. This is a contradiction.

By the above proof, we obtain the following:

Corollary 3.4. In the setting of Theorem 3.3 , let $\Omega$ be the open dense orbit in $\mathbb{X}$ and identify $T$ and $X$ by $\theta$. Then we have $T_{+} \subset \Omega$.

\section{Fundamental RePRESENTAtions AND PERFECT CRYSTALS}

4.1. Affine weights. Let $\mathfrak{g}$ be an affine Lie algebra and let the sets $\mathfrak{t},\left\{\alpha_{i}\right\}_{i \in I}$ and $\left\{\alpha_{i}^{\vee}\right\}_{i \in I}$ be as in 2.1. We take $\mathfrak{t}$ so that $\operatorname{dim} \mathfrak{t}=\sharp I+1$. Let $\delta \in Q_{+}$be a unique element satisfying

$$
\left\{\lambda \in Q \mid\left\langle\alpha_{i}^{\vee}, \lambda\right\rangle=0 \text { for any } i \in I\right\}=\mathbb{Z} \delta,
$$

and let $\mathbf{c} \in \sum_{i} \mathbb{Z}_{\geq 0} \alpha_{i}^{\vee} \subset \mathfrak{g}$ be a unique central element satisfying

$$
\left\{h \in Q^{\vee} \mid\left\langle h, \alpha_{i}\right\rangle=0 \text { for any } i \in I\right\}=\mathbb{Z} \mathbf{c} .
$$

We write $([\mathrm{Kac}, 6.1])$

$$
\mathbf{c}=\sum_{i} a_{i}^{\vee} \alpha_{i}^{\vee}, \quad \delta=\sum_{i} a_{i} \alpha_{i} .
$$

Let $($,$) be the non-degenerate W$-invariant symmetric bilinear form on $\mathfrak{t}^{*}$ normalized by $(\delta, \lambda)=\langle\mathbf{c}, \lambda\rangle$ for $\lambda \in \mathfrak{t}^{*}$. Let us set $\mathfrak{t}_{\mathrm{cl}}^{*}:=\mathfrak{t}^{*} / \mathbb{C} \delta$ and let $\mathrm{cl}: \mathfrak{t}^{*} \longrightarrow \mathfrak{t}_{\mathrm{cl}}^{*}$ be the canonical projection. Then we have $\mathfrak{t}_{\mathrm{cl}}^{*} \cong \bigoplus_{i}\left(\mathbb{C} \alpha_{i}^{\vee}\right)^{*}$. Set $\mathfrak{t}_{0}^{*}:=\left\{\lambda \in \mathfrak{t}^{*} \mid\langle\mathbf{c}, \lambda\rangle=0\right\}$, $\left(\mathfrak{t}_{\mathrm{cl}}^{*}\right)_{0}:=\mathrm{cl}\left(\mathfrak{t}_{0}^{*}\right)$. Then we have a positive-definite symmetric form on $\left(\mathfrak{t}_{\mathrm{cl}}^{*}\right)_{0}$ induced by the one on $\mathfrak{t}^{*}$. Let $\Lambda_{i} \in \mathfrak{t}_{\mathrm{cl}}^{*}(i \in I)$ be a weight such that $\left\langle\alpha_{i}^{\vee}, \Lambda_{j}\right\rangle=\delta_{i, j}$, which is called a fundamental weight. We choose $P$ so that $P_{\mathrm{cl}}:=\operatorname{cl}(P)$ coincides with $\bigoplus_{i \in I} \mathbb{Z} \Lambda_{i}$ and we call $P_{\mathrm{cl}}$ the classical weight lattice. 
4.2. Affinization. Let $U_{q}(\mathfrak{g})=\left\langle e_{i}, f_{i}, q^{h} \mid i \in I, h \in P\right\rangle$ be the quantum affine algebra associated with $P$ and $U_{q}^{\prime}(\mathfrak{g})=\left\langle e_{i}, f_{i}, q^{h} \mid i \in I, h \in\left(P_{\mathrm{cl}}\right)^{*}\right\rangle$ its subalgebra associated with $\left(P_{\mathrm{cl}}\right)^{*}$. Set $\operatorname{Mod}^{f}\left(\mathfrak{g}, P_{\mathrm{cl}}\right)$ the category of a finite-dimensional $U_{q}^{\prime}(\mathfrak{g})$ module $M$ with a weight decomposition $M=\bigoplus_{\lambda \in P_{\mathrm{cl}}} M_{\lambda}$.

Let $M$ be an object in $\operatorname{Mod}^{f}\left(\mathfrak{g}, P_{\mathrm{cl}}\right)$. For $l \in \mathbb{C}^{\times}$, define the $U_{q}^{\prime}(\mathfrak{g})$-module $M_{l}$ as follows: There exists a $\mathbb{C}$-linear bijective homomorphism $\Phi_{l}: M \longrightarrow M$, such that

$$
\begin{aligned}
& q^{h} \Phi_{l}(u)=\Phi_{l}\left(q^{h} u\right) \quad \text { for } h \in P_{\mathrm{cl}}^{*}, \\
& e_{i} \Phi_{l}(u)=l^{\delta_{i, 0}} \Phi_{l}\left(e_{i} u\right), \\
& f_{i} \Phi_{l}(u)=l^{-\delta_{i, 0}} \Phi_{l}\left(f_{i} u\right) .
\end{aligned}
$$

The module $M_{l}:=\Phi_{l}(M)$ is said to be the affinization of $M$ ( KMN1, K1]).

4.3. Fundamental representation $W\left(\varpi_{1}\right)_{l}$. Here we consider the following affine Lie algebras $\mathfrak{g}=A_{n}^{(1)}, B_{n}^{(1)}, D_{n}^{(1)}, A_{2 n-1}^{(2)}, D_{n+1}^{(2)}, A_{2 n}^{(2)}$. Let $\left\{\Lambda_{i} \mid i \in I\right\}$ be the set of fundamental weights as in $\$ 4.1$ Let $\varpi_{1}:=\Lambda_{1}-a_{1}^{\vee} \Lambda_{0}$ be the (level 0 ) fundamental weight, where $i=1$ is the node of the Dynkin diagram as below and $a_{i}^{\vee}$ is given in (4.1).

Let $W\left(\varpi_{1}\right)$ be the fundamental representation of $U_{q}^{\prime}(\mathfrak{g})$ (see [K1, Section 5]). By [K1, Theorem 5.17], $W\left(\varpi_{1}\right)$ is a finite-dimensional irreducible integrable $U_{q}^{\prime}(\mathfrak{g})$ module, an object in $\operatorname{Mod}^{f}\left(\mathfrak{g}, P_{\mathrm{lc}}\right)$ and has a global basis with a simple crystal. Thus, we can consider its affinization $W\left(\varpi_{1}\right)_{l}\left(l \in \mathbb{C}^{\times}\right)$specialized at $q=1$. Then we obtain a finite-dimensional $\mathfrak{g}$-module denoted also by $W\left(\varpi_{1}\right)_{l}$ in which we shall construct affine geometric crystals in the next section.

Note that $W\left(\varpi_{1}\right)_{l}$ is an irreducible $\mathfrak{g}$-module for any $\mathfrak{g}$ as above. But, for some $i$ and $\mathfrak{g}, W\left(\varpi_{i}\right)_{l}$ is not necessarily an irreducible $\mathfrak{g}$-module.

Let us see the explicit description of $W\left(\varpi_{1}\right)_{l}$ for $\mathfrak{g}=A_{n}^{(1)}, B_{n}^{(1)}, D_{n}^{(1)}, A_{2 n-1}^{(2)}$, $D_{n+1}^{(2)}, A_{2 n}^{(2)}$.

4.4. $A_{n}^{(1)}(n \geq 2)$. The Cartan matrix $\left(a_{i j}\right)_{i, j \in I}(I:=\{0,1, \ldots, n\})$ of type $A_{n}^{(1)}$ is

$$
a_{i j}= \begin{cases}2 & \text { if } i=j \\ -1 & \text { if } i \equiv j \pm 1 \quad \bmod n+1 \\ 0 & \text { otherwise. }\end{cases}
$$

We have $\mathbf{c}=\sum_{i \in I} \alpha_{i}^{\vee}$ and $\delta=\sum_{i \in I} \alpha_{i}$.

The basis of $W\left(\varpi_{1}\right)_{l}$ is $\left\{v_{1}, v_{2}, \cdots, v_{n+1}\right\}$, and we have

$$
\operatorname{wt}\left(v_{i}\right)=\Lambda_{i}-\Lambda_{i-1} \quad(i=1 \cdots, n+1),
$$

where we understand $\Lambda_{n+1}=\Lambda_{0}$. The explicit actions of the $f_{i}$ 's are

$$
\begin{array}{ll}
f_{i} v_{i}=v_{i+1} & (1 \leq i \leq n), \quad f_{0} v_{n+1}=l^{-1} v_{1}, \\
f_{i} v_{j}=0 & \text { otherwise }
\end{array}
$$

and the explicit actions of $e_{i}$ 's are

$$
\begin{array}{ll}
e_{i} v_{i+1}=v_{i} & (1 \leq i \leq n), \quad e_{0} v_{1}=l v_{n+1}, \\
e_{i} v_{j}=0 & \text { otherwise. }
\end{array}
$$


4.5. $B_{n}^{(1)}(n \geq 2)$. The Cartan matrix $\left(a_{i j}\right)_{i, j \in I}(I:=\{0,1, \ldots, n\})$ of type $B_{n}^{(1)}$ is

$$
a_{i j}= \begin{cases}2 & i=j, \\ -1 & |i-j|=1 \text { and }(i, j) \neq(0,1),(1,0),(n, n-1) \text { or }(i, j)=(0,2),(2,0), \\ -2 & (i, j)=(n, n-1), \\ 0 & \text { otherwise. }\end{cases}
$$

The Dynkin diagram is

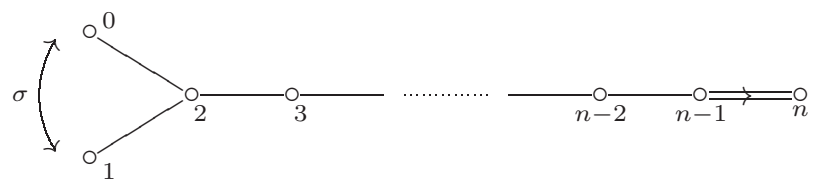

where $\sigma$ is the Dynkin diagram automorphism $\sigma: \alpha_{0} \leftrightarrow \alpha_{1}$ which will be needed later. We have

$$
\mathbf{c}=\alpha_{0}^{\vee}+\alpha_{1}^{\vee}+2 \sum_{i=2}^{n-1} \alpha_{i}^{\vee}+\alpha_{n}^{\vee}, \quad \delta=\alpha_{0}+\alpha_{1}+2 \sum_{i=2}^{n} \alpha_{i} .
$$

The basis of $W\left(\varpi_{1}\right)_{l}$ is $\left\{v_{1}, v_{2}, \cdots, v_{n}, v_{0}, v_{\bar{n}}, \cdots, v_{\overline{2}}, v_{\overline{1}}\right\}$, and we have

$$
\begin{aligned}
& \operatorname{wt}\left(v_{i}\right)=\Lambda_{i}-\Lambda_{i-1}, \quad \operatorname{wt}\left(v_{\bar{i}}\right)=\Lambda_{i-1}-\Lambda_{i} \quad(i \neq 0,2, n), \\
& \operatorname{wt}\left(v_{2}\right)=-\Lambda_{0}-\Lambda_{1}+\Lambda_{2}, \quad \operatorname{wt}\left(v_{\overline{2}}\right)=\Lambda_{0}+\Lambda_{1}-\Lambda_{2}, \\
& \operatorname{wt}\left(v_{n}\right)=2 \Lambda_{n}-\Lambda_{n-1}, \quad \operatorname{wt}\left(v_{\bar{n}}\right)=\Lambda_{n-1}-2 \Lambda_{n}, \quad \operatorname{wt}\left(v_{0}\right)=0 .
\end{aligned}
$$

The explicit forms of the actions by $f_{i}$ 's and $e_{i}$ 's are

$$
\begin{array}{llll}
f_{i} v_{i}=v_{i+1}, & f_{i} v_{\overline{i+1}}=v_{\bar{i}}(1 \leq i<n), & e_{i} v_{i+1}=v_{i}, & e_{i} v_{\bar{i}}=v_{\overline{i+1}}(1 \leq i<n), \\
f_{n} v_{n}=v_{0}, & f_{n} v_{0}=2 v_{\bar{n}}, & e_{n} v_{0}=2 v_{n}, & e_{n} v_{\bar{n}}=v_{0}, \\
f_{0} v_{\overline{2}}=l^{-1} v_{1}, & f_{0} v_{\overline{1}}=l^{-} v_{2}, & e_{0} v_{1}=l v_{\overline{2}}, & e_{0} v_{2}=l v_{\overline{1}}, \\
f_{i} v_{j}=0 & \text { otherwise, } & e_{i} v_{j}=0 & \text { otherwise, }
\end{array}
$$

4.6. $D_{n}^{(1)}(n \geq 4)$. The Cartan matrix $\left(a_{i j}\right)_{i, j \in I}(I:=\{0,1, \ldots, n\})$ of type $D_{n}^{(1)}$ is

$$
a_{i j}= \begin{cases}2 & i=j, \\ -1 & |i-j|=1 \text { and } 1 \leq i, j \leq n-1 \\ 0 & \text { otherwise. }\end{cases}
$$

The Dynkin diagram is

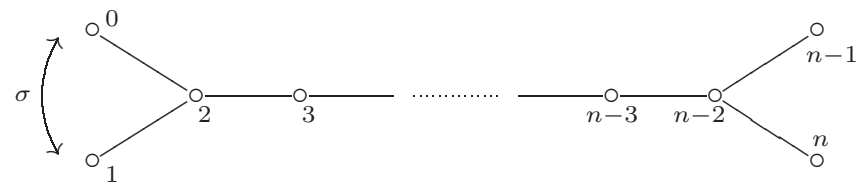

where $\sigma$ is the Dynkin diagram automorphism $\sigma: \alpha_{0} \leftrightarrow \alpha_{1}$ and $\sigma \alpha_{i}=\alpha_{i}$ for $i \neq 0,1$. We have

$$
\mathbf{c}=\alpha_{0}^{\vee}+\alpha_{1}^{\vee}+2 \sum_{i=2}^{n-2} \alpha_{i}^{\vee}+\alpha_{n-1}^{\vee}+\alpha_{n}^{\vee}, \quad \delta=\alpha_{0}+\alpha_{1}+2 \sum_{i=2}^{n-2} \alpha_{i}+\alpha_{n-1}+\alpha_{n} .
$$


The basis of $W\left(\varpi_{1}\right)_{l}$ is $\left\{v_{1}, v_{2}, \cdots, v_{n}, v_{\bar{n}}, \cdots, v_{\overline{2}}, v_{\overline{1}}\right\}$, and we have

$$
\begin{aligned}
& \operatorname{wt}\left(v_{i}\right)=\Lambda_{i}-\Lambda_{i-1}, \quad \operatorname{wt}\left(v_{\bar{i}}\right)=\Lambda_{i-1}-\Lambda_{i} \quad(i \neq 2, n-1), \\
& \operatorname{wt}\left(v_{2}\right)=-\Lambda_{0}-\Lambda_{1}+\Lambda_{2}, \quad \operatorname{wt}\left(v_{\overline{2}}\right)=\Lambda_{0}+\Lambda_{1}-\Lambda_{2}, \\
& \operatorname{wt}\left(v_{n-1}\right)=\Lambda_{n-1}+\Lambda_{n}-\Lambda_{n-1}, \quad \operatorname{wt}\left(v_{\overline{n-1}}\right)=\Lambda_{n-2}-\Lambda_{n-1}-\Lambda_{n} .
\end{aligned}
$$

The explicit forms of the actions by $f_{i}$ 's and $e_{i}$ 's are

$$
\begin{array}{llll}
f_{i} v_{i}=v_{i+1}, & f_{i} v_{\overline{i+1}}=v_{\bar{i}}(1 \leq i<n), & e_{i} v_{i+1}=v_{i}, & e_{i} v_{\bar{i}}=v_{\overline{i+1}}(1 \leq i<n), \\
f_{n} v_{n}=v_{\overline{n-1}}, & f_{n} v_{n-1}=v_{\bar{n}}, & e_{n} v_{\bar{n}}=v_{n-1}, & e_{n} v_{\overline{n-1}}=v_{n}, \\
f_{0} v_{\overline{2}}=l^{-1} v_{1}, & f_{0} v_{\overline{1}}=l^{-1} v_{2}, & e_{0} v_{1}=l v_{\overline{2}}, & e_{0} v_{2}=l v_{\overline{1}}, \\
f_{i} v_{j}=0 & \text { otherwise, } & e_{i} v_{j}=0 & \text { otherwise. }
\end{array}
$$

4.7. $A_{2 n-1}^{(2)}(n \geq 3)$. The Cartan matrix $\left(a_{i j}\right)_{i, j \in I}(I:=\{0,1, \ldots, n\})$ of type $A_{2 n-1}^{(2)}$ is

$$
a_{i j}= \begin{cases}2 & i=j, \\ -1 & |i-j|=1 \text { and } 1 \leq i, j \leq n-1 \text { or }(i, j)=(0,2),(2,0),(n, n-1), \\ -2 & (i, j)=(n-1, n), \\ 0 & \text { otherwise. }\end{cases}
$$

The Dynkin diagram is

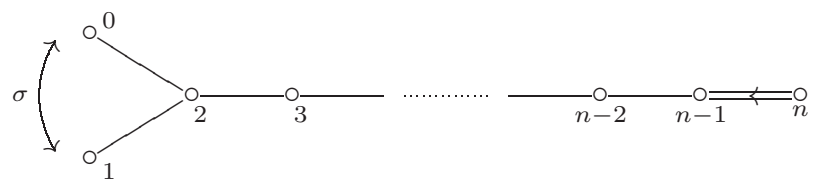

where $\sigma$ is the Dynkin diagram automorphism $\sigma: \alpha_{0} \leftrightarrow \alpha_{1}$. We have

$$
\mathbf{c}=\alpha_{0}^{\vee}+\alpha_{1}^{\vee}+2 \sum_{i=2}^{n} \alpha_{i}^{\vee}, \quad \delta=\alpha_{0}+\alpha_{1}+2 \sum_{i=2}^{n-1} \alpha_{i}+\alpha_{n} .
$$

The basis of $W\left(\varpi_{1}\right)_{l}$ is $\left\{v_{1}, v_{2}, \cdots, v_{n}, v_{\bar{n}}, \cdots, v_{\overline{2}}, v_{\overline{1}}\right\}$, and we have

$$
\begin{aligned}
& \operatorname{wt}\left(v_{i}\right)=\Lambda_{i}-\Lambda_{i-1}, \quad \operatorname{wt}\left(v_{\bar{i}}\right)=\Lambda_{i-1}-\Lambda_{i} \quad(i \neq 2), \\
& \operatorname{wt}\left(v_{2}\right)=-\Lambda_{0}-\Lambda_{1}+\Lambda_{2}, \quad \operatorname{wt}\left(v_{\overline{2}}\right)=\Lambda_{0}+\Lambda_{1}-\Lambda_{2} .
\end{aligned}
$$

The explicit forms of the actions by $f_{i}$ 's and $e_{i}$ 's are

$$
\begin{aligned}
& f_{i} v_{i}=v_{i+1}, \quad f_{i} v_{\overline{i+1}}=v_{\bar{i}} \quad(1 \leq i<n), \quad e_{i} v_{i+1}=v_{i}, \quad e_{i} v_{\bar{i}}=v_{\overline{i+1}} \quad(1 \leq i<n), \\
& f_{n} v_{n}=v_{\bar{n}}, \quad e_{n} v_{\bar{n}}=v_{n}, \\
& f_{0} v_{\overline{2}}=l^{-1} v_{1}, \quad f_{0} v_{\overline{1}}=l^{-1} v_{2}, \quad e_{0} v_{1}=l v_{\overline{2}}, \quad e_{0} v_{2}=l v_{\overline{1}}, \\
& f_{i} v_{j}=0 \quad \text { otherwise, } \quad e_{i} v_{j}=0 \quad \text { otherwise. }
\end{aligned}
$$

4.8. $D_{n+1}^{(2)}(n \geq 2)$. The Cartan matrix $\left(a_{i j}\right)_{i, j \in I}(I:=\{0,1, \ldots, n\})$ of type $D_{n+1}^{(2)}$ is

$$
a_{i j}= \begin{cases}2 & i=j, \\ -1 & |i-j|=1 \text { and }(i, j) \neq(0,1),(n, n-1) \\ -2 & (i, j)=(0,1),(n, n-1) \\ 0 & \text { otherwise. }\end{cases}
$$


The Dynkin diagram is

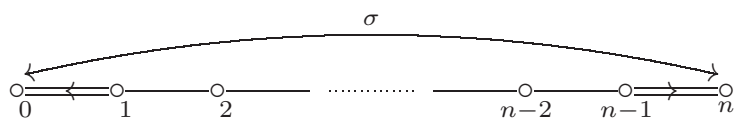

where $\sigma$ is the Dynkin diagram automorphism $\sigma: \alpha_{i} \leftrightarrow \alpha_{n-i}(i=0,1 \cdots, n)$. We have

$$
\mathbf{c}=\alpha_{0}^{\vee}+\alpha_{1}^{\vee}+2 \sum_{i=2}^{n-1} \alpha_{i}^{\vee}+\alpha_{n}^{\vee}, \quad \delta=\sum_{i=0}^{n} \alpha_{i} .
$$

The basis of $W\left(\varpi_{1}\right)_{l}$ is $\left\{v_{1}, v_{2}, \cdots, v_{n}, v_{0}, v_{\bar{n}}, \cdots, v_{\overline{2}}, v_{\overline{1}}, \phi\right\}$, and we have

$$
\begin{aligned}
& \operatorname{wt}\left(v_{i}\right)=\Lambda_{i}-\Lambda_{i-1}, \quad \quad \operatorname{wt}\left(v_{\bar{i}}\right)=\Lambda_{i-1}-\Lambda_{i} \quad(i \neq 0,1, n), \\
& \operatorname{wt}\left(v_{1}\right)=\Lambda_{1}-2 \Lambda_{0}, \quad \operatorname{wt}\left(v_{\overline{1}}\right)=2 \Lambda_{0}-\Lambda_{1}, \\
& \operatorname{wt}\left(v_{n}\right)=2 \Lambda_{n}-\Lambda_{n-1}, \quad \operatorname{wt}\left(v_{\bar{n}}\right)=\Lambda_{n-1}-2 \Lambda_{n}, \\
& \operatorname{wt}\left(v_{0}\right)=0, \quad \operatorname{wt}(\phi)=0
\end{aligned}
$$

The explicit forms of the actions by $f_{i}$ 's and $e_{i}$ 's are

$$
\begin{array}{llll}
f_{i} v_{i}=v_{i+1}, & f_{i} v_{\overline{i+1}}=v_{\bar{i}}(1 \leq i<n), & e_{i} v_{i+1}=v_{i}, & e_{i} v_{\bar{i}}=v_{\overline{i+1}} \quad(1 \leq i<n), \\
f_{n} v_{n}=v_{0}, & f_{n} v_{0}=2 v_{\bar{n}}, & e_{n} v_{0}=2 v_{n}, & e_{n} v_{\bar{n}}=v_{0}, \\
f_{0} v_{\overline{1}}=l^{-1} \phi, & f_{0} \phi=2 l^{-1} v_{1}, & e_{0} v_{1}=l \phi, & e_{0} \phi=2 l v_{\overline{1}}, \\
f_{i} v_{j}=0, \quad f_{i} \phi=0 & \text { otherwise, } & e_{i} v_{j}=0, \quad e_{i} \phi=0 \text { otherwise. }
\end{array}
$$

4.9. $A_{2 n}^{(2)}(n \geq 2)$. The Cartan matrix $\left(a_{i j}\right)_{i, j \in I}(I:=\{0,1, \ldots, n\})$ of type $A_{2 n}^{(2)}$ is

$$
a_{i j}= \begin{cases}2 & i=j, \\ -1 & |i-j|=1 \text { and }(i, j) \neq(0,1),(n-1, n), \\ -2 & (i, j)=(0,1),(n-1, n), \\ 0 & \text { otherwise. }\end{cases}
$$

Then the Dynkin diagram is

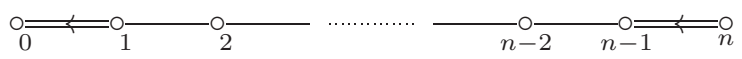

Note that there exists no Dynkin diagram automorphism in this case. We have

$$
\mathbf{c}=\alpha_{0}^{\vee}+2 \sum_{i=1}^{n} \alpha_{i}^{\vee}, \quad \delta=2 \sum_{i=0}^{n-1} \alpha_{i}+\alpha_{n} .
$$

In this case, we denote this type by $\left(A_{2 n}^{(2)}, \varpi_{1}\right)$ in order to distinguish it from the type $\left(A_{2 n}^{(2)} \dagger, \varpi_{1}\right)$. Then the basis of $W\left(\varpi_{1}\right)_{l}$ is $\left\{v_{1}, v_{2}, \cdots, v_{n}, v_{\bar{n}}, \cdots, v_{\overline{2}}, v_{\overline{1}}, \emptyset\right\}$, and we have

$$
\begin{aligned}
& \operatorname{wt}\left(v_{1}\right)=\Lambda_{1}-2 \Lambda_{0}, \quad \operatorname{wt}\left(v_{i}\right)=\Lambda_{i}-\Lambda_{i-1}, \quad \operatorname{wt}\left(v_{\bar{i}}\right)=\Lambda_{i-1}-\Lambda_{i} \quad(i=2, \ldots, n), \\
& \operatorname{wt}\left(v_{\overline{1}}\right)=2 \Lambda_{0}-\Lambda_{1}, \quad \operatorname{wt}(\emptyset)=0 .
\end{aligned}
$$


The explicit forms of the actions by $f_{i}$ 's and $e_{i}$ 's are

$$
\begin{array}{llll}
f_{i} v_{i}=v_{i+1}, & f_{i} v_{\overline{i+1}}=v_{\bar{i}}(1 \leq i<n), & e_{i} v_{i+1}=v_{i}, & e_{i} v_{\bar{i}}=v_{\overline{i+1}} \quad(1 \leq i<n), \\
f_{n} v_{n}=v_{\bar{n}}, & & e_{n} v_{n}=v_{n}, & \\
f_{0} v_{\overline{1}}=l^{-1} \emptyset, & f_{0} \emptyset=2 l^{-1} v_{1}, & e_{0} v_{1}=l \emptyset, & e_{0} \emptyset=2 l v_{\overline{1}}, \\
f_{i} v_{j}=0 & \text { otherwise }, & e_{i} v_{j}=0, & \text { otherwise. }
\end{array}
$$

4.10. $A_{2 n}^{(2) \dagger}(n \geq 2)$. The Cartan matrix $\left(a_{i j}\right)_{i, j \in I}(I:=\{0,1, \ldots, n\})$ of type $A_{2 n}^{(2)^{\dagger}}$ is

$$
a_{i j}= \begin{cases}2 & i=j, \\ -1 & |i-j|=1 \text { and }(i, j) \neq(1,0),(n, n-1), \\ -2 & (i, j)=(1,0),(n, n-1), \\ 0 & \text { otherwise. }\end{cases}
$$

Then the Dynkin diagram is

$$
\underset{0}{\Longrightarrow} \underset{1}{\mathrm{O}} \longrightarrow
$$

Note that there exists no Dynkin diagram automorphism in this case. We have

$$
\mathbf{c}=2 \sum_{i=0}^{n-1} \alpha_{i}^{\vee}+\alpha_{n}^{\vee}, \quad \delta=\alpha_{0}+2 \sum_{i=1}^{n} \alpha_{i} .
$$

In this case, we denote this type by $\left(A_{2 n}^{(2) \dagger}, \varpi_{1}\right)$ in order to distinguish it from the type $\left(A_{2 n}^{(2)}, \varpi_{1}\right)$. Then the basis of $W\left(\varpi_{1}\right)_{l}$ is $\left\{v_{1}, v_{2}, \cdots, v_{n}, v_{0}, v_{\bar{n}}, \cdots, v_{\overline{2}}, v_{\overline{1}}\right\}$, and we have

$$
\begin{aligned}
& \operatorname{wt}\left(v_{i}\right)=\Lambda_{i}-\Lambda_{i-1}, \quad \quad \operatorname{wt}\left(v_{\bar{i}}\right)=\Lambda_{i-1}-\Lambda_{i} \quad(i=1, \ldots, n-1), \\
& \operatorname{wt}\left(v_{n}\right)=2 \Lambda_{n}-\Lambda_{n-1}, \quad \quad \operatorname{wt}\left(v_{0}\right)=0, \quad \operatorname{wt}\left(v_{\overline{1}}\right)=\Lambda_{n-1}-2 \Lambda_{n} .
\end{aligned}
$$

The explicit forms of the actions by $f_{i}$ 's and $e_{i}$ 's are

$$
\begin{array}{llll}
f_{i} v_{i}=v_{i+1}, & f_{i} v_{\overline{i+1}}=v_{\bar{i}}(1 \leq i<n), & e_{i} v_{i+1}=v_{i}, & e_{i} v_{\bar{i}}=v_{\overline{i+1}} \quad(1 \leq i<n), \\
f_{n} v_{n}=v_{\overline{0}}, & f_{n} v_{0}=2 v_{\bar{n}}, & e_{n} v_{0}=2 v_{n}, & e_{n} v_{\bar{n}}=v_{0}, \\
f_{0} v_{\overline{1}}=l^{-1} v_{1}, & & e_{0} v_{1}=l v_{\overline{1}}, & \\
f_{i} v_{j}=0, & \text { otherwise, } & e_{i} v_{j}=0, & \text { otherwise. }
\end{array}
$$

4.11. Limit of perfect crystals. We review the limits of perfect crystals following KKM]. (See also KMN1, KMN2.)

Let $\mathfrak{g}$ be an affine Lie algebra, $P_{\mathrm{cl}}$ the classical weight lattice as above and for $l \in \mathbb{Z}_{>0}$ set $\left(P_{\mathrm{cl}}\right)_{l}^{+}:=\left\{\lambda \in P_{\mathrm{cl}} \mid\langle\mathbf{c}, \lambda\rangle=l,\left\langle\alpha_{i}^{\vee}, \lambda\right\rangle \geq 0\right\}$.

Definition 4.1. We say that a crystal $B$ is perfect of level $l$ if:

(i) $B \otimes B$ is connected as a crystal graph.

(ii) There exists $\lambda_{0} \in P_{\mathrm{cl}}$ such that

$$
\operatorname{wt}(B) \subset \lambda_{0}+\sum_{i \neq 0} \mathbb{Z}_{\leq 0} \operatorname{cl}\left(\alpha_{i}\right), \quad \sharp B_{\lambda_{0}}=1 .
$$

(iii) There exists a finite-dimensional $U_{q}^{\prime}(\mathfrak{g})$-module $V$ with a crystal pseudobase $B_{p s}$ such that $B \cong B_{p s} / \pm 1$. 
(iv) Set $\varepsilon(b):=\sum_{i} \varepsilon_{i}(b) \Lambda_{i}$ and $\varphi(b):=\sum_{i} \varphi_{i}(b) \Lambda_{i}$. For any $b \in B,\langle\mathbf{c}, \varepsilon(b)\rangle \geq l$ and the maps $\varepsilon, \varphi: B^{\min }:=\{b \in B \mid\langle c, \varepsilon(b)\rangle=l\} \longrightarrow\left(P_{\mathrm{cl}}^{+}\right)_{l}$ are bijective.

For an affine Lie algebra $\mathfrak{g}$, let $\left\{B_{l}(\mathfrak{g})\right\}_{l \geq 1}$ be a family of perfect crystals of level $l$ and set $J:=\left\{(l, b) \mid l>0, b \in B_{l}^{\min }\right\}$.

Definition 4.2. A crystal $B_{\infty}=B_{\infty}(\mathfrak{g})$ with an element $b_{\infty}$ is called the limit of $\left\{B_{l}\right\}_{l \geq 1}$ if:

(i) $\operatorname{wt}\left(b_{\infty}\right)=\varepsilon\left(b_{\infty}\right)=\varphi\left(b_{\infty}\right)=0$.

(ii) For any $(l, b) \in J$, there exists an embedding of crystals:

$$
\begin{aligned}
f_{(l, b)}: \quad T_{\varepsilon(b)} \otimes B_{l} \otimes T_{-\varphi(b)} & \hookrightarrow B_{\infty}, \\
t_{\varepsilon(b)} \otimes b \otimes t_{-\varphi(b)} & \mapsto b_{\infty} .
\end{aligned}
$$

(iii) $B_{\infty}=\bigcup_{(l, b) \in J} \operatorname{Im} f_{(l, b)}$.

As for the crystal $T_{\lambda}$, see Example 2.3.(iii). If the limit of a family $\left\{B_{l}\right\}$ exists, we say that $\left\{B_{l}\right\}$ is a coherent family of perfect crystals.

Remark. By the definition of perfect crystals, any perfect crystal is connected and then the limit of a coherent family of perfect crystals is also connected.

\section{Affine geOmetric CRYstals}

Following the method in $\mathrm{KNO}$, we shall construct the affine geometric crystal $\mathcal{V}(\mathfrak{g})_{l}\left(l \in \mathbb{C}^{\times}\right)$in the $\mathfrak{g}$-module $W\left(\varpi_{1}\right)_{l}$, the affinization of the fundamental representation $W\left(\varpi_{1}\right)$.

5.1. Translation $t\left(\tilde{\varpi}_{1}\right)$. For $\xi_{0} \in\left(\mathfrak{t}_{\mathrm{cl}}^{*}\right)_{0}$, let $t\left(\xi_{0}\right)$ be as in [K1, Section 4], that is,

$$
t\left(\xi_{0}\right)(\lambda):=\lambda+(\delta, \lambda) \xi-(\xi, \lambda) \delta-\frac{(\xi, \xi)}{2}(\delta, \lambda) \delta
$$

for $\xi \in \mathfrak{t}^{*}$ such that $\operatorname{cl}(\xi)=\xi_{0}$. Then $t\left(\xi_{0}\right)$ does not depend on the choice of $\xi$, and it is well defined.

Let $c_{i}^{\vee}$ be as follows $(\underline{\mathrm{K} 2})$ :

$$
c_{i}^{\vee}:=\max \left(1, \frac{2}{\left(\alpha_{i}, \alpha_{i}\right)}\right) .
$$

Then $t\left(m \varpi_{i}\right)$ belongs to the extended Weyl group $\widetilde{W}$ if and only if $m \in c_{i}^{\vee} \mathbb{Z}$. Setting $\widetilde{\varpi}_{i}:=c_{i}^{\vee} \varpi_{i}(i \in I), t\left(\widetilde{\varpi}_{1}\right)$ is expressed as follows (see e.g. [KOTUY]):

$$
t\left(\widetilde{\varpi}_{1}\right)= \begin{cases}\iota\left(s_{n} s_{n-1} \cdots s_{2} s_{1}\right) & A_{n}^{(1)} \text { case, } \\ \iota\left(s_{1} \cdots s_{n}\right)\left(s_{n-1} \cdots s_{2} s_{1}\right) & B_{n}^{(1)}, A_{2 n-1}^{(2)} \text { cases, } \\ \left(s_{0} s_{1} \cdots s_{n}\right)\left(s_{n-1} \cdots s_{2} s_{1}\right) & C_{n}^{(1)}, D_{n+1}^{(2)} \text { cases, } \\ \iota\left(s_{1} \cdots s_{n}\right)\left(s_{n-2} \cdots s_{2} s_{1}\right) & D_{n}^{(1)} \text { case, } \\ \left(s_{0} s_{1} \cdots s_{n}\right)\left(s_{n-1} \cdots s_{2} s_{1}\right) & A_{2 n}^{(2)}, A_{2 n}^{(2) \dagger} \text { cases, }\end{cases}
$$

where $\iota$ is the Dynkin diagram automorphism

$$
\iota= \begin{cases}\sigma & \mathfrak{g}=A_{n}^{(1)}, B_{n}^{(1)}, A_{2 n-1}^{(2)}, \\ \alpha_{0} \leftrightarrow \alpha_{1} \text { and } \alpha_{n-1} \leftrightarrow \alpha_{n} & \mathfrak{g}=D_{n}^{(1)} .\end{cases}
$$

Now, we know that each $t\left(\widetilde{\varpi}_{1}\right)$ is in the form $w_{1}$ or $\iota \cdot w_{1}$ for some $w_{1} \in W$, e.g., $w_{1}=s_{n} \cdots s_{1}$ for $A_{n}^{(1)}, w_{1}=\left(s_{1} \cdots s_{n}\right)\left(s_{n-1} \cdots s_{1}\right)$ for $B_{n}^{(1)}$, etc., $\ldots$ 
In the case $A_{2 n}^{(2)}\left(\right.$ resp. $\left.\mathfrak{g}=A_{2 n}^{(2) \dagger}\right), \eta:=\operatorname{wt}\left(v_{\bar{n}}\right)=\Lambda_{n-1}-\Lambda_{n}\left(\right.$ resp. $\left.\Lambda_{n-1}-2 \Lambda_{n}\right)$ is a unique weight of $W\left(\varpi_{1}\right)_{l}$ which satisfies $\left\langle\alpha_{i}^{\vee}, \eta\right\rangle \geq 0$ for $i \neq n$. For this $\eta$ we have

$$
t(\eta)=\left(s_{n} s_{n-1} \cdots s_{1}\right)\left(s_{0} s_{1} \cdots s_{n-1}\right)=: w_{2},
$$

which will be used later.

5.2. Affine geometric crystals in $W\left(\varpi_{1}\right)_{l}$. Let $\sigma$ be the Dynkin diagram automorphism as in 4.44 .8 and let $w_{1}=s_{i_{1}} \cdots s_{i_{k}}$ be as in the previous subsection. Let $H$ be an element in $\mathfrak{t}$ such that

$$
\alpha_{i}(H)= \begin{cases}1 & \text { if } i=1 \text { and } \mathfrak{g} \neq D_{n+1}^{(2)}, A_{2 n}^{(2)}, A_{2 n}^{(2)^{\dagger}} \\ 2 & \text { if } i=1 \text { and } \mathfrak{g}=D_{n+1}^{(2)}, A_{2 n}^{(2)}, A_{2 n}^{(2)^{\dagger}} \\ -1 & \text { if } i=0 \text { and } \mathfrak{g} \neq D_{n+1}^{(2)}, A_{2 n}^{(2)}, A_{2 n}^{(2)^{\dagger}} \\ -2 & \text { if } i=0 \text { and } \mathfrak{g}=D_{n+1}^{(2)}, A_{2 n}^{(2)}, A_{2 n}^{(2)^{\dagger}} \\ 0 & \text { otherwise. }\end{cases}
$$

Set

$$
\mathcal{V}(\mathfrak{g})_{l}:=\left\{v\left(x_{1}, \ldots, x_{k}\right):=Y_{i_{1}}\left(x_{1}\right) \cdots Y_{i_{k}}\left(x_{k}\right) l^{H} v_{1} \mid x_{1}, \ldots, x_{k} \in \mathbb{C}^{\times}\right\} \subset W\left(\varpi_{1}\right)_{l} .
$$

Let $\mathfrak{g}_{0} \subset \mathfrak{g}$ (resp. $\left.G_{0} \subset G\right)$ be a simple Lie algebra (resp. simple algebraic group) corresponding to the index set $I_{0}:=I \backslash\{0\}$. Since the vector $v_{1}$ satisfies $x_{i}(c) v_{1}=v_{1}$ for any $i \in I_{0}$, the actions of $e_{i}^{c}\left(i \in I_{0}\right)$ on $v(x)$ and $Y_{i_{1}}\left(x_{1}\right) \cdots Y_{i_{k}}\left(x_{k}\right) \cdot l^{H}$ coincide with each other. Therefore, $\mathcal{V}(\mathfrak{g})_{l}$ has a $G_{0}$-geometric crystal structure the same as that of $B_{i_{1} \cdots i_{k}}^{-} \cdot l^{H}$ (see 2.3$)$, Moreover, $\left(\mathbb{C}^{\times}\right)^{k} \rightarrow \mathcal{V}(\mathfrak{g})_{l}$ given by $\left(x_{1}, \ldots, x_{k}\right) \mapsto$ $v\left(x_{1}, \ldots, x_{k}\right)$ is a birational map. We shall define a $G$-geometric crystal structure on $\mathcal{V}(\mathfrak{g})_{l}$ by using the Dynkin diagram automorphism $\sigma$ except for $A_{2 n}^{(2)}$. This $\sigma$ induces an automorphism of $W\left(\varpi_{1}\right)_{l}$, which is denoted by $\sigma_{l}: W\left(\varpi_{1}\right)_{l} \longrightarrow W\left(\varpi_{1}\right)_{l}$. The following theorems are analogous results to Theorem 5.1 and 5.2 in [KNO].

Theorem 5.1. (i) Case $\mathfrak{g} \neq A_{2 n}^{(2)}, A_{2 n}^{(2)^{\dagger}}$. For $x=\left(x_{1} \cdots, x_{k}\right) \in\left(\mathbb{C}^{\times}\right)^{k}$, there exist a unique $y=\left(y_{1}, \ldots, y_{k}\right) \in\left(\mathbb{C}^{\times}\right)^{k}$ and a positive rational function $a(x)$ such that

$$
v(y)=a(x) \sigma_{l}(v(x)), \quad \varepsilon_{\sigma(i)}(v(y))=\varepsilon_{i}(v(x)) \quad \text { if } i, \sigma(i) \neq 0 .
$$

(ii) Case $\mathfrak{g}=A_{2 n}^{(2)}$ (resp. $A_{2 n}^{(2)^{\dagger}}$ ). Associated with $w_{1}$ and $w_{2}$ as in the previous section, we define

$$
\begin{aligned}
\mathcal{V}(\mathfrak{g})_{l}:= & \left\{v_{1}(x)=Y_{0}\left(x_{0}\right) Y_{1}\left(x_{1}\right) \cdots Y_{n}\left(x_{n}\right) Y_{n-1}\left(\bar{x}_{n-1}\right) \cdots Y_{1}\left(\bar{x}_{1}\right) l^{H} v_{1} \mid x_{i}, \bar{x}_{i} \in \mathbb{C}^{\times}\right\}, \\
\mathcal{V}_{2}(\mathfrak{g})_{l}:= & \left\{v_{2}(y)=Y_{n}\left(y_{n}\right) \cdots Y_{1}\left(y_{1}\right) Y_{0}\left(y_{0}\right) Y_{1}\left(\bar{y}_{1}\right) \cdots Y_{n-1}\left(\bar{y}_{n-1}\right) l^{H^{\prime}} v_{\bar{n}} \mid y_{i}, \bar{y}_{i} \in \mathbb{C}^{\times}\right\}, \\
& \text {where } \left.\alpha_{0}\left(H^{\prime}\right)=2 \text { (resp. } \alpha_{0}\left(H^{\prime}\right)=2\right), \alpha_{n}\left(H^{\prime}\right)=-4\left(\text { resp. } \alpha_{n}\left(H^{\prime}\right)=-1\right) \\
& \text { and } \left.\alpha_{i}\left(H^{\prime}\right)=0 \text { otherwise. (Note that } \operatorname{wt}\left(v_{1}\right)(H)=\operatorname{wt}\left(v_{\bar{n}}\right)\left(H^{\prime}\right) .\right) \text { For any } \\
& x \in\left(\mathbb{C}^{\times}\right)^{2 n} \text { there exist a unique } y \in\left(\mathbb{C}^{\times}\right)^{2 n} \text { and a rational function } a(x) \\
& \text { such that } v_{2}(y)=a(x) v_{1}(x) .
\end{aligned}
$$


Now, using this theorem, we define the rational mapping

$$
\begin{array}{ll}
\bar{\sigma}: \mathcal{V}(\mathfrak{g})_{l} \longrightarrow \mathcal{V}(\mathfrak{g})_{l}, & \bar{\sigma}: \mathcal{V}(\mathfrak{g})_{l} \longrightarrow \mathcal{V}_{2}(\mathfrak{g})_{l}, \\
v(x) \mapsto v(y) \quad\left(\mathfrak{g} \neq A_{2 n}^{(2)}, A_{2 n}^{(2)^{\dagger}}\right), & v_{1}(x) \mapsto v_{2}(y) \quad\left(\mathfrak{g}=A_{2 n}^{(2)}, A_{2 n}^{(2)}{ }^{\dagger}\right),
\end{array}
$$

Theorem 5.2. The rational mapping $\bar{\sigma}$ is birational. If we define a $\mathfrak{g}_{0}$-geometric crystal structure on $\mathcal{V}(\mathfrak{g})_{l}$ by the one on $B_{i_{1} \cdots i_{k}}^{-} \cdot l^{H}\left(w_{1}=s_{i_{1}} \cdots s_{i_{k}}\right)$ as in 2.3 and a rational $\mathbb{C}^{\times}$-action $e_{0}: \mathbb{C}^{\times} \times \mathcal{V}(\mathfrak{g})_{l} \rightarrow \mathcal{V}(\mathfrak{g})_{l}$ and rational functions $\mathrm{wt}_{0}$ and $\varepsilon_{0}$ on $\mathcal{V}(\mathfrak{g})_{l}$ by

$$
\left\{\begin{array}{r}
e_{0}^{c}:=\bar{\sigma}^{-1} \circ e_{\sigma(0)}^{c} \circ \bar{\sigma}, \quad \varepsilon_{0}:=\varepsilon_{\sigma(0)} \circ \bar{\sigma}, \quad \gamma_{0}:=\gamma_{\sigma(0)} \circ \bar{\sigma}, \\
\text { for } \mathfrak{g} \neq A_{2 n}^{(2)}, A_{2 n}^{(2)}, \\
e_{0}^{c}:=\bar{\sigma}^{-1} \circ e_{0}^{c} \circ \bar{\sigma}, \quad \varepsilon_{0}:=\varepsilon_{0} \circ \bar{\sigma}, \quad \gamma_{0}:=\gamma_{0} \circ \bar{\sigma}, \\
\text { for } \mathfrak{g}=A_{2 n}^{(2)}, A_{2 n}^{(2)}{ }^{\dagger} .
\end{array}\right.
$$

then $\left(\mathcal{V}(\mathfrak{g})_{l},\left\{e_{i}\right\}_{i \in I},\left\{\gamma_{i}\right\}_{i \in I},\left\{\varepsilon_{i}\right\}_{i \in I}\right)$ turns out to be an affine $\mathfrak{g}$-geometric crystal.

Remark. In the case $\mathfrak{g}=A_{2 n}^{(2)}$ and $A_{2 n}^{(2)^{\dagger}}, \mathcal{V}_{2}(\mathfrak{g})_{l}$ has a $\mathfrak{g}_{I \backslash\{n\} \text {-geometric crystal }}$ structure. Thus, $e_{0}, \gamma_{0}, \varepsilon_{0}$ are well defined on $\mathcal{V}_{2}(\mathfrak{g})_{l}$.

The following lemma shows Theorem 5.2 partially.

Lemma 5.3. Suppose that $\mathfrak{g} \neq A_{2 n}^{(2)}, A_{2 n}^{(2)}{ }^{\dagger}$. If there exists $\bar{\sigma}$ as above and

$$
e_{\sigma(i)}^{c}=\bar{\sigma} \circ e_{i}^{c} \circ \bar{\sigma}^{-1}, \quad \gamma_{i}=\gamma_{\sigma(i)} \circ \bar{\sigma}, \quad \varepsilon_{i}=\varepsilon_{\sigma(i)} \circ \bar{\sigma},
$$

for $i \neq \sigma^{-1}(0), 0$, then we obtain

(i) $e_{0}^{c_{1}} e_{i}^{c_{2}}=e_{i}^{c_{2}} e_{0}^{c_{1}} \quad$ if $a_{0 i}=a_{i 0}=0$,

$e_{0}^{c_{1}} e_{i}^{c_{1} c_{2}} e_{0}^{c_{2}}=e_{i}^{c_{2}} e_{0}^{c_{1} c_{2}} e_{i}^{c_{2}} \quad$ if $a_{0 i} a_{i 0}=1$,

$e_{0}^{c_{1}} e_{i}^{c_{1}^{2} c_{2}} e_{0}^{c_{1} c_{2}} e_{i}^{c_{2}}=e_{i}^{c_{2}} e_{0}^{c_{1} c_{2}} e_{i}^{c_{1}^{2} c_{2}} e_{0}^{c_{1}} \quad$ if $a_{0 i}=-2, a_{i 0}=-1$,

$e_{0}^{c_{2}} e_{i}^{c_{1} c_{2}} e_{0}^{c_{1}^{2} c_{2}} e_{i}^{c_{1}}=e_{i}^{c_{1}} e_{0}^{c_{1}^{2} c_{2}} e_{i}^{c_{1} c_{2}} e_{0}^{c_{2}} \quad$ if $a_{0 i}=-1, a_{i 0}=-2$.

(ii) $\gamma_{0}\left(e_{i}^{c}(v(x))\right)=c^{a_{i 0}} \gamma_{0}(v(x))$ and $\gamma_{i}\left(e_{0}^{c}(v(x))\right)=c^{a_{0 i}} \gamma_{i}(v(x))$.

(iii) $\varepsilon_{0}\left(e_{0}^{c}(v(x))\right)=c^{-1} \varepsilon_{0}(v(x))$.

Proof. For example, we have

$$
\begin{aligned}
\gamma_{0}\left(e_{i}^{c}(v(x))\right) & =\gamma_{\sigma(0)}\left(\bar{\sigma} e_{i}^{c} \bar{\sigma}^{-1}(\bar{\sigma}(v(x)))\right) \\
& =\gamma_{\sigma(0)}\left(e_{\sigma(i)}^{c}(\bar{\sigma}(v(x)))\right)=c^{a_{\sigma(i), \sigma(0)}} \gamma_{\sigma(0)}(\bar{\sigma}(v(x))) \\
& =c^{a_{i, 0}} \gamma_{0}(v(x)),
\end{aligned}
$$

where we use $a_{\sigma(i), \sigma(0)}=a_{i 0}$ in the last equality. The other assertions are obtained similarly.

In the rest of this section, we shall prove Theorem 5.1 and Theorem 5.2 in caseby-case methods.

5.3. $A_{n}^{(1)}$-case $(n \geq 2)$. We have $w_{1}:=s_{n} s_{n-1} \cdots s_{2} s_{1}$, and

$$
\mathcal{V}\left(A_{n}^{(1)}\right)_{l}:=\left\{Y_{n}\left(x_{n}\right) \cdots Y_{2}\left(x_{2}\right) Y_{1}\left(x_{1}\right) l^{H} v_{1} \mid x_{i} \in \mathbb{C}^{\times}\right\} \subset W\left(\varpi_{1}\right)_{l} .
$$


Since $y_{i}\left(\frac{1}{c}\right)=\exp \left(\frac{f_{i}}{c}\right)=1+c^{-1} f_{i}$ on $W\left(\varpi_{1}\right), v(x)=Y_{n}\left(x_{n}\right) \cdots Y_{2}\left(x_{2}\right) Y_{1}\left(x_{1}\right) l^{H} v_{1}$ is explicitly written as

$$
v(x)=v\left(x_{1}, \ldots, x_{n}\right)=l^{m}\left(\sum_{i=1}^{n} x_{i} v_{i}+v_{n+1}\right),
$$

where $m=\varpi_{1}(H)$. Let $\sigma: \alpha_{k} \mapsto \alpha_{k+1}(k \in I)$ be the Dynkin diagram automorphism for $A_{n}^{(1)}$, which gives rise to the automorphism $\sigma_{l}: W\left(\varpi_{1}\right)_{l} \rightarrow W\left(\varpi_{1}\right)_{l}$. We have

Then, we obtain

$$
\sigma_{l}\left(v_{i}\right)= \begin{cases}v_{i+1} & i \neq n+1 \\ l^{-1} v_{1} & i=n+1\end{cases}
$$

$$
\sigma_{l}(v(x))=l^{m}\left(l^{-1} v_{1}+\sum_{i=1}^{n} x_{i} v_{i+1}\right) .
$$

Then the equation $v(y)=a(x) \sigma_{l}(v(x))$, i.e.,

$$
\sum_{i=1}^{n} y_{i} v_{i}+v_{n+1}=a(x)\left(l^{-1} v_{1}+\sum_{i=1}^{n} x_{i} v_{i+1}\right)
$$

is solved by

$$
a(x)=\frac{1}{x_{n}}, \quad y_{1}=\frac{1}{l x_{n}}, \quad y_{i}=\frac{x_{i-1}}{x_{n}}(i=2, \ldots, n),
$$

that is,

$$
\bar{\sigma}\left(v\left(x_{1} \cdots, x_{n}\right)\right)=v\left(\frac{1}{l x_{n}}, \frac{x_{1}}{x_{n}}, \ldots, \frac{x_{n-1}}{x_{n}}\right) .
$$

The $A_{n}$-geometric crystal structure on $\mathcal{V}\left(A_{n}^{(1)}\right)_{l}$ induced from the one on $B_{w_{1}}^{-} \cdot l^{H}$ is given by:

$$
\begin{gathered}
\gamma_{1}(v(x))=\frac{l x_{1}^{2}}{x_{2}}, \quad \gamma_{i}(v(x))=\frac{x_{i}^{2}}{x_{i-1} x_{i+1}} \quad(i=2, \ldots, n-1), \quad \gamma_{n}(v(x))=\frac{x_{n}^{2}}{x_{n-1}}, \\
\varepsilon_{i}(v(x))=\frac{x_{i+1}}{x_{i}} \quad(i=1, \ldots, n-1), \quad \varepsilon_{n}(v(x))=\frac{1}{x_{n}} .
\end{gathered}
$$

Then we have

$$
\varepsilon_{i+1}(\bar{\sigma}(v(x)))= \begin{cases}\frac{x_{i+1}}{x_{i}} & \text { if } i=1 \cdots, n-2, \\ \frac{x_{n}}{x_{n-1}} & \text { if } i=n-1,\end{cases}
$$

which implies $\varepsilon_{\sigma(i)}(\bar{\sigma}(v(x)))=\varepsilon_{i}(v(x))$, and then we completed the proof of Theorem 5.1 for $A_{n}^{(1)}$.

Now, we define $e_{0}^{c}, \gamma_{0}$ and $\varepsilon_{0}$ by

$$
e_{0}^{c}:=\bar{\sigma}^{-1} \circ e_{1}^{c} \circ \bar{\sigma}, \quad \gamma_{0}:=\gamma_{1} \circ \bar{\sigma}, \quad \varepsilon_{0}:=\varepsilon_{1} \circ \bar{\sigma} .
$$

Their explicit forms are

$$
\begin{aligned}
& e_{0}^{c}(v(x))=v\left(\frac{x_{1}}{c}, \frac{x_{2}}{c}, \ldots, \frac{x_{n}}{c}\right), \\
& \gamma_{0}(v(x))=\frac{1}{l x_{1} x_{n}}, \quad \varepsilon_{0}(v(x))=l x_{1} .
\end{aligned}
$$


Thus, we can check (5.7) easily, and then Lemma 5.3 reduces the proof of Theorem 5.2 to the statements:

$$
\begin{aligned}
& e_{0}^{c_{1}} e_{n}^{c_{1} c_{2}} e_{0}^{c_{2}}=e_{n}^{c_{2}} e_{0}^{c_{1} c_{2}} e_{n}^{c_{1}}, \\
& \gamma_{0}\left(e_{n}^{c}(v(x))\right)=c^{-1} \gamma_{0}(v(x)), \quad \gamma_{n}\left(e_{0}^{c}(v(x))\right)=c^{-1} \gamma_{n}(v(x)) .
\end{aligned}
$$

These are immediate from (5.10)-(5.15). Thus, we obtain Theorem 5.2 for $A_{n}^{(1)}$.

Let us introduce the $A_{n}^{(1)}$-geometric crystal $\mathcal{B}_{L}\left(A_{n}^{(1)}\right)\left(L \in \mathbb{C}^{\times}\right)$([KOTY $]$:

$$
\begin{aligned}
& \mathcal{B}_{L}\left(A_{n}^{(1)}\right):=\left\{l=\left(l_{1}, \ldots, l_{n}, l_{n+1}\right) \in\left(\mathbb{C}^{\times}\right)^{n+1} \mid l_{1} \cdots l_{n+1}=L\right\}, \\
& e_{i}^{c}(l)=\left(\cdots, c l_{i}, c^{-1} l_{i+1}, \ldots\right), \quad \gamma_{i}(l)=\frac{l_{i}}{l_{i+1}}, \quad \varepsilon_{i}(l)=l_{i+1} \quad(i=0,1, \ldots, n),
\end{aligned}
$$

where we understand $l_{0}=l_{n+1}$.

We have $\mathcal{B}_{L}\left(A_{n}^{(1)}\right) \cong \mathcal{V}\left(A_{n}^{(1)}\right)_{L}$. Indeed, defining $\phi: \mathcal{B}_{L}\left(A_{n}^{(1)}\right) \rightarrow \mathcal{V}\left(A_{n}^{(1)}\right)_{L}$ by $\phi\left(l_{1}, \cdots, l_{n+1}\right)=v\left(\frac{l_{1}}{L}, \frac{l_{1} l_{2}}{L}, \cdots, \frac{l_{1} \cdots l_{n}}{L}\right)$, it is easy to see that $\phi$ is an isomorphism of geometric crystals.

5.4. $B_{n}^{(1)}$-case $(n \geq 2)$. We have $w_{1}=s_{1} \cdots s_{n-1} s_{n} s_{n-1} \cdots s_{1}$, and

$$
\mathcal{V}\left(B_{n}^{(1)}\right)_{l}:=\left\{v(x)=Y_{1}\left(x_{1}\right) \cdots Y_{n}\left(x_{n}\right) Y_{n-1}\left(\bar{x}_{n-1}\right) \cdots Y_{1}\left(\bar{x}_{1}\right) l^{H} v_{1} \mid x_{i}, \bar{x}_{i} \in \mathbb{C}^{\times}\right\} .
$$

It follows from the explicit description of $W\left(\varpi_{1}\right)_{l}$ as in 4.5 that

$$
\begin{gathered}
v\left(x_{1}, \ldots, x_{n}, \bar{x}_{n-1}, \ldots, \bar{x}_{1}\right)=l^{m}\left\{\left(\sum_{i=1}^{n} \xi_{i}(x) v_{i}\right)+x_{n} v_{0}+\left(\sum_{i=2}^{n} x_{i-1} v_{\bar{i}}\right)+v_{\overline{1}}\right\}, \\
\text { where } m:=\varpi_{1}(H) \quad \text { and } \quad \xi_{i}(x):= \begin{cases}\frac{x_{1} \bar{x}_{1} \bar{x}_{i-1}+x_{i} \bar{x}_{i}}{x_{i-1}} & i \neq 1, n, \\
\frac{x_{n-1} \bar{x}_{n-1}+x_{n}^{2}}{x_{n-1}} & i=n .\end{cases}
\end{gathered}
$$

The automorphism $\sigma_{l}: W\left(\varpi_{1}\right)_{l} \rightarrow W\left(\varpi_{1}\right)_{l}$ is given as

$$
\sigma_{l} v_{1}=l v_{\overline{1}}, \quad \sigma_{l} v_{\overline{1}}=l^{-1} v_{1}, \sigma_{l} v_{k}=v_{k} \text { otherwise. }
$$

Then we have

$$
\sigma_{l}(v(x))=l^{m}\left\{l^{-1} v_{1}+\left(\sum_{i=2}^{n} \xi_{i}(x) v_{i}\right)+x_{n} v_{0}+\left(\sum_{i=2}^{n} x_{i-1} v_{\bar{i}}\right)+l x_{1} \bar{x}_{1} v_{\overline{1}}\right\},
$$

The equation $v(y)=a(x) \sigma_{l}(v(x))\left(x, y \in\left(\mathbb{C}^{\times}\right)^{2 n-1}\right)$ has a unique solution:

$$
\begin{gathered}
a(x)=\frac{1}{l x_{1} \bar{x}_{1}}, \quad y_{i}=a(x) x_{i}=\frac{x_{i}}{l x_{1} \bar{x}_{1}} \quad(1 \leq i \leq n), \\
\bar{y}_{i}=a(x) \bar{x}_{i}=\frac{\bar{x}_{i}}{l x_{1} \bar{x}_{1}} \quad(1 \leq i<n) .
\end{gathered}
$$

Hence we have the rational mapping

$$
\bar{\sigma}(v(x)):=v(y)=v\left(\frac{x_{1}}{l x_{1} \bar{x}_{1}}, \frac{x_{2}}{l x_{1} \bar{x}_{1}}, \cdots, \frac{x_{n}}{l x_{1} \bar{x}_{1}}, \frac{\bar{x}_{n-1}}{l x_{1} \bar{x}_{1}}, \cdots, \frac{\bar{x}_{1}}{l x_{1} \bar{x}_{1}}\right) .
$$

By the explicit form of $\bar{\sigma}$ in (5.20), we have $\bar{\sigma}^{2}=\mathrm{id}$, which means that the morphism $\bar{\sigma}$ is birational. In this case, the second condition in Theorem 5.1 is trivial since $\sigma(i)=i$ if $i, \sigma(i) \neq 0$. Thus, the proof of Theorem 5.1 in this case is completed.

Now, we set $e_{0}^{c}:=\bar{\sigma} \circ e_{1}^{c} \circ \bar{\sigma}, \gamma_{0}:=\gamma_{1} \circ \bar{\sigma}$ and $\varepsilon_{0}:=\varepsilon_{1} \circ \bar{\sigma}$. 
The explicit forms of $e_{i}, \varepsilon_{i}$ and $\gamma_{i}$ are

$$
\begin{aligned}
& e_{0}^{c}: \quad x_{1} \mapsto \quad x_{1} \frac{c x_{1} \bar{x}_{1}+x_{2} \bar{x}_{2}}{c\left(x_{1} \bar{x}_{1}+x_{2} \bar{x}_{2}\right)} \quad x_{i} \mapsto \frac{x_{i}}{c}(2 \leq i \leq n), \\
& \bar{x}_{1} \mapsto \quad \bar{x}_{1} \frac{x_{1} \bar{x}_{1}+x_{2} \bar{x}_{2}}{c x_{1} \bar{x}_{1}+x_{2} \bar{x}_{2}}, \quad \bar{x}_{i} \mapsto \frac{\bar{x}_{i}}{c}(2 \leq i \leq n-1), \\
& e_{i}^{c}: \quad x_{i} \mapsto \quad x_{i} \frac{c x_{i} \bar{x}_{i}+x_{i+1} \bar{x}_{i+1}}{x_{i} \bar{x}_{i}+x_{i+1} \bar{x}_{i+1}}, \quad \bar{x}_{i} \mapsto \bar{x}_{i} \frac{c\left(x_{i} \bar{x}_{i}+x_{i+1} \bar{x}_{i+1}\right)}{c x_{i} \bar{x}_{i}+x_{i+1} \bar{x}_{i+1}}, \\
& x_{j} \mapsto \quad x_{j}, \quad \bar{x}_{j} \mapsto \bar{x}_{j}(j \neq i) \quad(1 \leq i<n-1), \\
& e_{n-1}^{c}: \quad x_{n-1} \mapsto \quad x_{n-1} \frac{c x_{n-1} \bar{x}_{n-1}+x_{n}^{2}}{x_{n-1} \bar{x}_{n-1}+x_{n}^{2}}, \quad \bar{x}_{n-1} \mapsto \bar{x}_{n-1} \frac{c\left(x_{n-1} \bar{x}_{n-1}+x_{n}^{2}\right)}{c x_{n-1} \bar{x}_{n-1}+x_{n}^{2}}, \\
& x_{j} \mapsto \quad x_{j}, \quad \bar{x}_{j} \mapsto \bar{x}_{j}(j \neq n-1), \\
& e_{n}^{c}: \quad x_{n} \mapsto \quad c x_{n}, \quad x_{j} \mapsto x_{j} \quad \bar{x}_{j} \mapsto \bar{x}_{j}(j \neq n), \\
& \varepsilon_{0}(v(x))=\frac{l\left(x_{1} \bar{x}_{1}+x_{2} \bar{x}_{2}\right)}{x_{1}}, \quad \varepsilon_{1}(v(x))=\frac{1}{x_{1}}\left(1+\frac{x_{2} \bar{x}_{2}}{x_{1} \bar{x}_{1}}\right), \\
& \varepsilon_{i}(v(x))=\frac{x_{i-1}}{x_{i}}\left(1+\frac{x_{i+1} \bar{x}_{i+1}}{x_{i} \bar{x}_{i}}\right)(2 \leq i \leq n-2), \\
& \varepsilon_{n-1}(v(x))=\frac{x_{n-2}}{x_{n-1}}\left(1+\frac{x_{n}^{2}}{x_{n-1} \bar{x}_{n-1}}\right), \varepsilon_{n}(v(x))=\frac{x_{n-1}}{x_{n}}, \\
& \gamma_{0}(v(x))=\frac{1}{l x_{2} \bar{x}_{2}}, \gamma_{1}(v(x))=\frac{l\left(x_{1} \bar{x}_{1}\right)^{2}}{x_{2} \bar{x}_{2}} \\
& \gamma_{i}(v(x))=\frac{\left(x_{i} \bar{x}_{i}\right)^{2}}{x_{i-1} \bar{x}_{i-1} x_{i+1} \bar{x}_{i+1}}(2 \leq i \leq n-2), \\
& \gamma_{n-1}(v(x))=\frac{\left(x_{n-1} \bar{x}_{n-1}\right)^{2}}{x_{n-2} \bar{x}_{n-2} x_{n}^{2}}, \quad \gamma_{n}(v(x))=\frac{x_{n}^{2}}{x_{n-1} \bar{x}_{n-1}} .
\end{aligned}
$$

Since $\sigma(i)=i$ for $i \neq 0,1$, the condition (5.7) in Lemma 5.3 can be easily seen by (5.20) and by the explicit form of $e_{i}, \gamma_{i}$ and $\varepsilon_{i}(i \in I)$. Thus, in order to prove Theorem 5.2, it suffices to show that

$$
\begin{aligned}
& e_{0}^{c_{1}} e_{1}^{c_{2}}=e_{1}^{c_{2}} e_{0}^{c_{1}}, \\
& \gamma_{0}\left(e_{1}^{c}(v(x))\right)=\gamma_{0}(v(x)), \quad \gamma_{1}\left(e_{0}^{c}(v(x))\right)=\gamma_{1}(v(x)) .
\end{aligned}
$$

It follows from the explicit formula above that

$$
\begin{aligned}
e_{0}^{c_{1}} e_{1}^{c_{2}}(v(x)) & =e_{1}^{c_{2}} e_{0}^{c_{1}}(v(x)) \\
& =v\left(x_{1} \frac{c_{1} c_{2} x_{1} \bar{x}_{1}+x_{2} \bar{x}_{2}}{c_{1}\left(x_{1} \bar{x}_{1}+x_{2} \bar{x}_{2}\right)}, \frac{x_{2}}{c_{1}}, \cdots, \frac{\bar{x}_{2}}{c_{1}}, \bar{x}_{1} \frac{c_{2}\left(x_{1} \bar{x}_{1}+x_{2} \bar{x}_{2}\right)}{c_{1} c_{2} x_{1} \bar{x}_{1}+x_{2} \bar{x}_{2}}\right),
\end{aligned}
$$

which implies (5.21). We get (5.22) immediately from the formula above and we complete the proof of Theorem 5.2 for $B_{n}^{(1)}$. 
5.5. $D_{n}^{(1)}$-case $(n \geq 4)$. We have $w_{1}=s_{1} s_{2} \cdots s_{n-1} s_{n} s_{n-2} s_{n-3} \cdots s_{2} s_{1}$, and

$$
\begin{gathered}
\mathcal{V}\left(D_{n}^{(1)}\right)_{l}:=\left\{v(x)=Y_{1}\left(x_{1}\right) \cdots Y_{n-1}\left(x_{n-1}\right) Y_{n}\left(x_{n}\right) Y_{n-2}\left(\bar{x}_{n-2}\right) \cdots\right. \\
\left.Y_{1}\left(\bar{x}_{1}\right) l^{H} v_{1} \mid x_{i}, \bar{x}_{i} \in \mathbb{C}^{\times}\right\} .
\end{gathered}
$$

It follows from the explicit form of $W\left(\varpi_{1}\right)_{l}$ in 4.6 that $y_{i}\left(c^{-1}\right)=\exp \left(c^{-1} f_{i}\right)=$ $1+c^{-1} f_{i}$ on $W\left(\varpi_{1}\right)$. Thus, we have

$$
\begin{aligned}
& v(x)=l^{m}\left\{\left(\sum_{i=1}^{n-1} \xi_{i}(x) v_{i}\right)+x_{n} v_{n}+\left(\sum_{i=2}^{n} x_{i-1} v_{\bar{i}}\right)+v_{\overline{1}}\right\}, \\
& \text { where } m:=\varpi_{1}(H), \quad \xi_{i}(x):= \begin{cases}\frac{x_{1} \bar{x}_{1}}{\frac{x_{i-1} \bar{x}_{i-1}+x_{i} \bar{x}_{i}}{x_{i-1}}} & i \neq 1, n-1, \\
\frac{x_{n-2} \bar{x}_{n-2}+x_{n-1} x_{n}}{x_{n-2}} & i=n-1 .\end{cases}
\end{aligned}
$$

The automorphism $\sigma_{l}: W\left(\varpi_{1}\right)_{l} \rightarrow W\left(\varpi_{1}\right)_{l}$ is given as

$$
\sigma_{l} v_{1}=l v_{\overline{1}}, \quad \sigma_{l} v_{\overline{1}}=l^{-1} v_{1}, \quad \sigma_{l} v_{k}=v_{k} \quad \text { otherwise. }
$$

Then we have

$$
\sigma_{l}(v(x))=l^{m}\left\{l^{-1} v_{1}+\left(\sum_{i=2}^{n} \xi_{i}(x) v_{i}\right)+x_{n} v_{n}+\left(\sum_{i=1}^{n-1} x_{i-1} v_{\bar{i}}\right)+l \xi_{1} v_{\overline{1}}\right\}
$$

Then the equation $v(y)=a(x) \sigma_{l}(v(x))\left(x, y \in\left(\mathbb{C}^{\times}\right)^{2 n-2}\right)$ has the following unique solution:

$$
\begin{gathered}
a(x)=\frac{1}{l x_{1} \bar{x}_{1}}, \quad y_{i}=a(x) x_{i}=\frac{x_{i}}{l x_{1} \bar{x}_{1}} \quad(1 \leq i \leq n), \\
\bar{y}_{i}=a(x) \bar{x}_{i}=\frac{\bar{x}_{i}}{l x_{1} \bar{x}_{1}} \quad(1 \leq i \leq n-2) .
\end{gathered}
$$

We define the rational mapping $\bar{\sigma}: \mathcal{V}\left(D_{n}^{(1)}\right)_{l} \longrightarrow \mathcal{V}\left(D_{n}^{(1)}\right)_{l}$ by

$$
\bar{\sigma}(v(x))=v\left(\frac{x_{1}}{l x_{1} \bar{x}_{1}}, \frac{x_{2}}{l x_{1} \bar{x}_{1}}, \cdots, \frac{x_{n}}{l x_{1} \bar{x}_{1}}, \frac{\bar{x}_{n-2}}{l x_{1} \bar{x}_{1}}, \cdots, \frac{\bar{x}_{1}}{l x_{1} \bar{x}_{1}}\right) .
$$

It is immediate from (5.24) that $\bar{\sigma}^{2}=\mathrm{id}$, which implies the birationality of the morphism $\bar{\sigma}$. In this case, the second condition in Theorem 5.1 is trivial since $\sigma(i)=i$ if $i, \sigma(i) \neq 0$. Thus, the proof of Theorem 5.1 for $D_{n}^{(1)}$ is completed. 
Now, we set $e_{0}^{c}:=\bar{\sigma} \circ e_{1}^{c} \circ \bar{\sigma}, \gamma_{0}:=\gamma_{1} \circ \bar{\sigma}$ and $\varepsilon_{0}:=\varepsilon_{1} \circ \bar{\sigma}$. The explicit forms of $e_{i}, \varepsilon_{i}$ and $\gamma_{i}$ are

$$
\begin{aligned}
& e_{0}^{c}: x_{1} \mapsto x_{1} \frac{c x_{1} \bar{x}_{1}+x_{2} \bar{x}_{2}}{c\left(x_{1} \bar{x}_{1}+x_{2} \bar{x}_{2}\right)}, \quad x_{i} \mapsto \frac{x_{i}}{c}(2 \leq i \leq n), \\
& \bar{x}_{1} \mapsto \bar{x}_{1} \frac{x_{1} \bar{x}_{1}+x_{2} \bar{x}_{2}}{c x_{1} \bar{x}_{1}+x_{2} \bar{x}_{2}}, \quad \bar{x}_{i} \mapsto \frac{\bar{x}_{i}}{c}(2 \leq i \leq n-2), \\
& e_{i}^{c}: x_{i} \mapsto x_{i} \frac{c x_{i} \bar{x}_{i}+x_{i+1} \bar{x}_{i+1}}{x_{i} \bar{x}_{i}+x_{i+1} \bar{x}_{i+1}}, \quad \bar{x}_{i} \mapsto \bar{x}_{i} \frac{c\left(x_{i} \bar{x}_{i}+x_{i+1} \bar{x}_{i+1}\right)}{c x_{i} \bar{x}_{i}+x_{i+1} \bar{x}_{i+1}}, \\
& x_{j} \mapsto x_{j}, \quad \bar{x}_{j} \mapsto \bar{x}_{j} \quad(j \neq i), \quad(1 \leq i \leq n-3), \\
& e_{n-2}^{c}: x_{n-2} \mapsto x_{n-2} \frac{c x_{n-2} \bar{x}_{n-2}+x_{n-1} x_{n}}{x_{n-2} \bar{x}_{n-2}+x_{n-1} x_{n}}, \quad \bar{x}_{n-2} \mapsto \bar{x}_{n-2} \frac{c\left(x_{n-2} \bar{x}_{n-2}+x_{n-1} x_{n}\right)}{c x_{n-2} \bar{x}_{n-2}+x_{n-1} x_{n}}, \\
& x_{j} \mapsto x_{j}, \quad \bar{x}_{j} \mapsto \bar{x}_{j} \quad(j \neq n-2), \\
& e_{n-1}^{c}: x_{n-1} \mapsto c x_{n-1}, \quad x_{j} \mapsto x_{j}, \quad \bar{x}_{j} \mapsto \bar{x}_{j} \quad(j \neq n-1), \\
& e_{n}^{c}: x_{n} \mapsto c x_{n}, \quad x_{j} \mapsto x_{j}, \quad \bar{x}_{j} \mapsto \bar{x}_{j} \quad(j \neq n), \\
& \varepsilon_{0}(v(x))=\frac{l\left(x_{1} \bar{x}_{1}+x_{2} \bar{x}_{2}\right)}{x_{1}}, \quad \varepsilon_{1}(v(x))=\frac{1}{x_{1}}\left(1+\frac{x_{2} \bar{x}_{2}}{x_{1} \bar{x}_{1}}\right), \\
& \varepsilon_{i}(v(x))=\frac{x_{i-1}}{x_{i}}\left(1+\frac{x_{i+1} \bar{x}_{i+1}}{x_{i} \bar{x}_{i}}\right) \quad(2 \leq i \leq n-3), \\
& \varepsilon_{n-2}(v(x))=\frac{x_{n-3}}{x_{n-2}}\left(1+\frac{x_{n-1} x_{n}}{x_{n-2} \bar{x}_{n-2}}\right), \quad \varepsilon_{n-1}(v(x))=\frac{x_{n-2}}{x_{n-1}}, \quad \varepsilon_{n}(v(x))=\frac{x_{n-2}}{x_{n}}, \\
& \gamma_{0}(v(x))=\frac{1}{l x_{2} \bar{x}_{2}}, \gamma_{1}(v(x))=\frac{l\left(x_{1} \bar{x}_{1}\right)^{2}}{x_{2} \bar{x}_{2}}, \\
& \gamma_{i}(v(x))=\frac{\left(x_{i} \bar{x}_{i}\right)^{2}}{x_{i-1} \bar{x}_{i-1} x_{i+1} \bar{x}_{i+1}}(2 \leq i \leq n-3), \\
& \gamma_{n-2}(v(x))=\frac{\left(x_{n-2} \bar{x}_{n-2}\right)^{2}}{x_{n-3} \bar{x}_{n-3} x_{n-1} x_{n}}, \quad \gamma_{n-1}(v(x))=\frac{x_{n-1}^{2}}{x_{n-2} \bar{x}_{n-2}}, \\
& \gamma_{n}(v(x))=\frac{x_{n}^{2}}{x_{n-2} \bar{x}_{n-2}} .
\end{aligned}
$$

By these formulas, we can show Theorem 5.2 for $D_{n}^{(1)}$ similarly to the one for $B_{n}^{(1)}$.

5.6. $A_{2 n-1}^{(2)}$-case $(n \geq 3)$. We have $w_{1}=s_{1} s_{2} \cdots s_{n} s_{n-1} \cdots s_{2} s_{1}$, and

$$
\mathcal{V}\left(A_{2 n-1}^{(2)}\right)_{l}:=\left\{v(x):=Y_{1}\left(x_{1}\right) \cdots Y_{n}\left(x_{n}\right) Y_{n-1}\left(\bar{x}_{n-1}\right) \cdots Y_{1}\left(\bar{x}_{1}\right) l^{H} v_{1} \mid x_{i}, \bar{x}_{i} \in \mathbb{C}^{\times}\right\} .
$$

In this case, $y_{i}\left(c^{-1}\right)=\exp \left(c^{-1} f_{i}\right)=1+c^{-1} f_{i}$ on $W\left(\varpi_{1}\right)$, and we have

$$
\begin{gathered}
v\left(x_{1}, \ldots, x_{n}, \bar{x}_{n-1}, \ldots, \bar{x}_{1}\right)=l^{m}\left\{\left(\sum_{i=1}^{n} \xi_{i} v_{i}\right)+\left(\sum_{i=2}^{n} x_{i-1} v_{\bar{i}}\right)+v_{\overline{1}}\right\}, \\
\text { where } m:=\varpi_{1}(H), \quad \xi_{i}:= \begin{cases}\frac{x_{1} \bar{x}_{1}}{\frac{x_{i-1} \bar{x}_{i-1}+x_{i} \bar{x}_{i}}{x_{i-1}}} & i \neq 1, n, \\
\frac{x_{n-1} \bar{x}_{n-1}+x_{n}}{x_{n-1}} & i=n .\end{cases}
\end{gathered}
$$

The automorphism $\sigma_{l}: W\left(\varpi_{1}\right)_{l} \rightarrow W\left(\varpi_{1}\right)_{l}$ is given as

$$
\sigma_{l} v_{1}=l v_{\overline{1}}, \quad \sigma_{l} v_{\overline{1}}=l^{-1} v_{1}, \sigma_{l} v_{k}=v_{k} \quad \text { otherwise. }
$$


Then we have

$$
\sigma_{l}(v(x))=l^{m}\left\{l^{-1} v_{1}+\left(\sum_{i=2}^{n} \xi_{i} v_{i}\right)+\left(\sum_{i=2}^{n} x_{i-1} v_{\bar{i}}\right)+l x_{1} \bar{x}_{1} v_{\overline{1}}\right\},
$$

Solving $v(y)=a(x) \sigma_{l}(v(x))\left(x, y \in\left(\mathbb{C}^{\times}\right)^{2 n-1}\right)$, we obtain a unique solution:

$$
\begin{gathered}
a(x)=\frac{1}{l x_{1} \bar{x}_{1}}, y_{i}=a(x) x_{i}=\frac{x_{i}}{l x_{1} \bar{x}_{1}}, \\
\bar{y}_{i}=a(x) \bar{x}_{i}=\frac{\bar{x}_{i}}{l x_{1} \bar{x}_{1}}(1 \leq i \leq n-1), y_{n}=a(x)^{2} x_{n}=\frac{x_{n}}{\left(l x_{1} \bar{x}_{1}\right)^{2}} .
\end{gathered}
$$

Here we have

$$
\bar{\sigma}(v(x))=v\left(\frac{x_{1}}{l x_{1} \bar{x}_{1}}, \frac{x_{2}}{l x_{1} \bar{x}_{1}}, \cdots, \frac{x_{n}}{\left(l x_{1} \bar{x}_{1}\right)^{2}}, \frac{\bar{x}_{n-1}}{x_{1} \bar{x}_{1}}, \cdots, \frac{\bar{x}_{1}}{x_{1} \bar{x}_{1}}\right) .
$$

By the explicit form of $\bar{\sigma}$ in (5.26), we have $\bar{\sigma}^{2}=$ id, which means that the morphism $\bar{\sigma}$ is birational. In this case, the second condition in Theorem 5.1 is trivial since $\sigma(i)=i$ if $i, \sigma(i) \neq 0$. Thus, the proof of Theorem 5.1 for $A_{2 n-1}^{(2)}$ is completed.

Now, we set $e_{0}^{c}:=\bar{\sigma} \circ e_{1}^{c} \circ \bar{\sigma}, \gamma_{0}:=\gamma_{1} \circ \bar{\sigma}$ and $\varepsilon_{0}:=\varepsilon_{1} \circ \bar{\sigma}$. The explicit forms of $e_{i}, \varepsilon_{i}$ and $\gamma_{i}$ are

$$
\begin{aligned}
& e_{0}^{c}: x_{1} \mapsto x_{1} \frac{c x_{1} \bar{x}_{1}+x_{2} \bar{x}_{2}}{c\left(x_{1} \bar{x}_{1}+x_{2} \bar{x}_{2}\right)}, \quad x_{i} \mapsto \frac{x_{i}}{c}(2 \leq i \leq n-1), \quad x_{n} \mapsto \frac{x_{n}}{c^{2}} \\
& \bar{x}_{1} \mapsto \bar{x}_{1} \frac{x_{1} \bar{x}_{1}+x_{2} \bar{x}_{2}}{c x_{1} \bar{x}_{1}+x_{2} \bar{x}_{2}}, \quad \bar{x}_{i} \mapsto \frac{\bar{x}_{i}}{c}(2 \leq i \leq n-1), \\
& e_{i}^{c}: x_{i} \mapsto x_{i} \frac{c x_{i} \bar{x}_{i}+x_{i+1} \bar{x}_{i+1}}{x_{i} \bar{x}_{i}+x_{i+1} \bar{x}_{i+1}}, \quad \bar{x}_{i} \mapsto \bar{x}_{i} \frac{c\left(x_{i} \bar{x}_{i}+x_{i+1} \bar{x}_{i+1}\right)}{c x_{i} \bar{x}_{i}+x_{i+1} \bar{x}_{i+1}}, \\
& x_{j} \mapsto x_{j}, \quad \bar{x}_{j} \mapsto \bar{x}_{j} \quad(j \neq i) \quad(1 \leq i<n-1), \\
& e_{n-1}^{c}: x_{n-1} \mapsto x_{n-1} \frac{c x_{n-1} \bar{x}_{n-1}+x_{n}}{x_{n-1} \bar{x}_{n-1}+x_{n}}, \quad \bar{x}_{n-1} \mapsto \bar{x}_{n-1} \frac{c\left(x_{n-1} \bar{x}_{n-1}+x_{n}\right)}{c x_{n-1} \bar{x}_{n-1}+x_{n}}, \\
& x_{j} \mapsto x_{j}, \quad \bar{x}_{j} \mapsto \bar{x}_{j} \quad(j \neq n-1), \\
& e_{n}^{c}: x_{n} \mapsto c x_{n}, \quad x_{j} \mapsto x_{j}, \quad \bar{x}_{j} \mapsto \bar{x}_{j} \quad(j \neq n) . \\
& \varepsilon_{0}(v(x))=\frac{l\left(x_{1} \bar{x}_{1}+x_{2} \bar{x}_{2}\right)}{x_{1}}, \quad \varepsilon_{1}(v(x))=\frac{1}{x_{1}}\left(1+\frac{x_{2} \bar{x}_{2}}{x_{1} \bar{x}_{1}}\right), \\
& \varepsilon_{i}(v(x))=\frac{x_{i-1}}{x_{i}}\left(1+\frac{x_{i+1} \bar{x}_{i+1}}{x_{i} \bar{x}_{i}}\right) \quad(2 \leq i \leq n-2), \\
& \varepsilon_{n-1}(v(x))=\frac{x_{n-2}}{x_{n-1}}\left(1+\frac{x_{n}}{x_{n-1} \bar{x}_{n-1}}\right), \quad \varepsilon_{n}(v(x))=\frac{x_{n-1}^{2}}{x_{n}}, \\
& \gamma_{0}(v(x))=\frac{1}{l x_{2} \bar{x}_{2}}, \quad \gamma_{1}(v(x))=\frac{l\left(x_{1} \bar{x}_{1}\right)^{2}}{x_{2} \bar{x}_{2}}, \quad \gamma_{i}=\frac{\left(x_{i} \bar{x}_{i}\right)^{2}}{x_{i-1} \bar{x}_{i-1} x_{i+1} \bar{x}_{i+1}} \quad(2 \leq i \leq n-2) \text {, } \\
& \gamma_{n-1}(v(x))=\frac{\left(x_{n-1} \bar{x}_{n-1}\right)^{2}}{x_{n-2} \bar{x}_{n-2} x_{n}}, \quad \gamma_{n}(v(x))=\frac{x_{n}^{2}}{\left(x_{n-1} \bar{x}_{n-1}\right)^{2}} .
\end{aligned}
$$

We can show Theorem 5.2 for $A_{2 n-1}^{(2)}$ similarly to the one for $B_{n}^{(1)}$. 
5.7. $D_{n+1}^{(2)}$-case $(n \geq 2)$. We have $w_{1}=s_{0} s_{1} \cdots s_{n} s_{n-1} \cdots s_{2} s_{1}$, and

$$
\begin{aligned}
\mathcal{V}\left(D_{n+1}^{(2)}\right)_{l}:=\left\{v(x):=Y_{0}\left(x_{0}\right) Y_{1}\left(x_{1}\right)\right. & \cdots Y_{n}\left(x_{n}\right) Y_{n-1}\left(\bar{x}_{n-1}\right) \cdots \\
& \left.Y_{1}\left(\bar{x}_{1}\right) l^{H} v_{1} \mid x_{i}, \bar{x}_{i} \in \mathbb{C}^{\times}\right\} .
\end{aligned}
$$

It follows from the explicit description of $W\left(\varpi_{1}\right)_{l}$ as in 4.8 that on $W\left(\varpi_{1}\right)_{l}$ we have

$$
y_{i}\left(c^{-1}\right)=\exp \left(c^{-1} f_{i}\right)= \begin{cases}1+c^{-1} f_{i} & i \neq 0, n, \\ 1+c^{-1} f_{i}+\frac{1}{2 c^{2}} f_{i}^{2} & i=0, n .\end{cases}
$$

Then we have

$$
v(x)=l^{m}\left\{\left(\sum_{i=1}^{n} \xi_{i}(x) v_{i}\right)+x_{n} v_{0}+l^{-1} x_{0} \phi+\left(\sum_{i=2}^{n} x_{i-1} v_{\bar{i}}\right)+x_{0}^{2} v_{\overline{1}}\right\}
$$

where

$$
m:=\varpi_{1}(H), \quad \xi_{i}(x):= \begin{cases}\frac{l^{-2} x_{0}^{2}+x_{1} \bar{x}_{1}}{x_{0}^{2}} & i=1, \\ \frac{x_{i-1} \bar{x}_{i-1}+x_{i} \bar{x}_{i}}{x_{i-1}} & i \neq 1, n, \\ \frac{x_{n-1} \bar{x}_{n-1}+x_{n}^{2}}{x_{n-1}} & i=n .\end{cases}
$$

The automorphism $\sigma_{l}: W\left(\varpi_{1}\right)_{l} \rightarrow W\left(\varpi_{1}\right)_{l}$ is given as

$$
\begin{aligned}
& \sigma_{l} v_{1}=l v_{\bar{n}}, \quad \sigma_{l} v_{\overline{1}}=l^{-1} v_{n}, \quad \sigma_{l} v_{n}=l v_{\overline{1}}, \quad \sigma_{l} v_{\bar{n}}=l^{-1} v_{1}, \\
& \sigma_{l} v_{0}=\phi, \quad \sigma_{l} \phi=v_{0}, \quad \sigma_{l} v_{i+1}=v_{\overline{n-i}}, \quad \sigma_{l} v_{\overline{i+1}}=v_{n-i} \quad(1 \leq i<n-1) .
\end{aligned}
$$

Then we have

$$
\begin{aligned}
\sigma_{l}(v(x))=l^{m}\left\{l^{-1} x_{n-1} v_{1}+\left(\sum_{i=2}^{n-1} x_{n-i} v_{i}\right)+l^{-1} x_{0}^{2} v_{n}+l \xi_{n} v_{\bar{n}}\right. & \\
& \left.+\left(\sum_{i=2}^{n-1} \xi_{n-i+1} v_{\bar{i}}\right)+l \xi_{1} v_{\bar{n}}+x_{n} \phi+x_{0} v_{0}\right\},
\end{aligned}
$$

Solving $v(y)=a(x) \sigma_{l}(v(x))\left(x, y \in\left(\mathbb{C}^{\times}\right)^{2 n}\right)$, we get a unique solution:

$$
\begin{aligned}
& a(x)=\frac{x_{n-1} \bar{x}_{n-1}+x_{n}^{2}}{l x_{n-1} x_{n}^{2}}, \\
& y_{0}=l a(x) x_{n}=\frac{x_{n-1} \bar{x}_{n-1}+x_{n}^{2}}{x_{n-1} x_{n}}, \\
& y_{i}=\frac{\left(x_{n-i-1} \bar{x}_{n-i-1}+x_{n-i} \bar{x}_{n-i}\right)\left(x_{n-1} \bar{x}_{n-1}+x_{n}^{2}\right)}{l x_{n-i-1} x_{n-1} x_{n}^{2}} \quad(1 \leq i<n), \\
& y_{n-1}=\frac{\left(l^{-2} x_{0}^{2}+x_{1} \bar{x}_{1}\right)\left(x_{n-1} \bar{x}_{n-1}+x_{n}^{2}\right)}{x_{0}^{2} x_{n-1} x_{n}^{2}}, \\
& y_{n}=\frac{x_{0}\left(x_{n-1} \bar{x}_{n-1}+x_{n}^{2}\right)}{l^{2} x_{n-1} x_{n}^{2}}, \\
& \bar{y}_{i}=\frac{\left(x_{n-1} \bar{x}_{n-1}+x_{n}^{2}\right) x_{n-i-1} x_{n-i} \bar{x}_{n-i}}{l\left(x_{n-i-1} \bar{x}_{n-i-1}+x_{n-i} \bar{x}_{n-i}\right) x_{n-1} x_{n}^{2}} \quad(1 \leq i \leq n-2), \\
& \bar{y}_{n-1}=\frac{\left(x_{n-1} \bar{x}_{n-1}+x_{n}^{2}\right) x_{0}^{2} x_{1} \bar{x}_{1}}{\left(x_{0}^{2}+l^{2} x_{1} \bar{x}_{1}\right) x_{n-1} x_{n}^{2}} .
\end{aligned}
$$


Then we have the rational mapping $\bar{\sigma}: \mathcal{V}\left(D_{n+1}^{(2)}\right)_{l} \longrightarrow \mathcal{V}\left(D_{n+1}^{(2)}\right)_{l}$ defined by $v(x) \mapsto$ $v(y)$. The explicit forms of $\varepsilon_{i}(1 \leq i \leq n)$ are as follows:

$$
\begin{aligned}
\varepsilon_{1}(v(x)) & =\frac{x_{0}^{2}}{x_{1}}\left(1+\frac{x_{2} \bar{x}_{2}}{x_{1} \bar{x}_{1}}\right), \quad \varepsilon_{n}(v(x))=\frac{x_{n-1}}{x_{n}}, \\
\varepsilon_{i}(v(x)) & =\frac{x_{i-1}}{x_{i}}\left(1+\frac{x_{i+1} \bar{x}_{i+1}}{x_{i} \bar{x}_{i}}\right) \quad(2 \leq i \leq n-2), \\
\varepsilon_{n-1}(v(x)) & =\frac{x_{n-2}}{x_{n-1}}\left(1+\frac{x_{n}^{2}}{x_{n-1} \bar{x}_{n-1}}\right) .
\end{aligned}
$$

Then we get easily that $\varepsilon_{n-i}(v(y))=\varepsilon_{i}(v(x))(1 \leq i \leq n-1)$, which finishes the proof of Theorem 5.1 for $D_{n+1}^{(2)}$.

Let us define $e_{0}^{c}:=\bar{\sigma} \circ e_{n}^{c} \circ \bar{\sigma}\left(\bar{\sigma}^{2}=\mathrm{id}\right), \gamma_{0}:=\gamma_{n} \circ \bar{\sigma}$ and $\varepsilon_{0}:=\varepsilon_{n} \circ \bar{\sigma}$. The explicit forms of $e_{i}, \gamma_{i}$ and $\varepsilon_{0}$ are

$$
\begin{aligned}
& e_{0}^{c}: \quad x_{0} \mapsto \quad x_{0} \frac{c^{2} x_{0}^{2}+l^{2} x_{1} \bar{x}_{1}}{c\left(x_{0}^{2}+l^{2} x_{1} \bar{x}_{1}\right)}, \quad x_{i} \mapsto x_{i} \frac{c^{2} x_{0}^{2}+l^{2} x_{1} \bar{x}_{1}}{c^{2}\left(x_{0}^{2}+l^{2} x_{1} \bar{x}_{1}\right)}(1 \leq i \leq n), \\
& \bar{x}_{i} \mapsto \quad \bar{x}_{i} \frac{c^{2} x_{0}^{2}+l^{2} x_{1} \bar{x}_{1}}{c^{2}\left(x_{0}^{2}+l^{2} x_{1} \bar{x}_{1}\right)} \quad(1 \leq i \leq n-1), \\
& e_{i}^{c}: \quad x_{i} \mapsto \quad x_{i} \frac{c x_{i} \bar{x}_{i}+x_{i+1} \bar{x}_{i+1}}{x_{i} \bar{x}_{i}+x_{i+1} \bar{x}_{i+1}}, \quad \bar{x}_{i} \mapsto \bar{x}_{i} \frac{c\left(x_{i} \bar{x}_{i}+x_{i+1} \bar{x}_{i+1}\right)}{c x_{i} \bar{x}_{i}+x_{i+1} \bar{x}_{i+1}}, \\
& x_{j} \mapsto \quad x_{j}, \quad \bar{x}_{j} \mapsto \bar{x}_{j} \quad(j \neq i), \quad(1 \leq i<n-1), \\
& e_{n-1}^{c}: \quad x_{n-1} \mapsto \quad x_{n-1} \frac{c x_{n-1} \bar{x}_{n-1}+x_{n}^{2}}{x_{n-1} \bar{x}_{n-1}+x_{n}^{2}}, \quad \bar{x}_{n-1} \mapsto \bar{x}_{n-1} \frac{c\left(x_{n-1} \bar{x}_{n-1}+x_{n}^{2}\right)}{c x_{n-1} \bar{x}_{n-1}+x_{n}^{2}}, \\
& x_{j} \mapsto \quad x_{j}, \quad \bar{x}_{j} \mapsto \bar{x}_{j} \quad(j \neq n-1), \\
& x_{n} \mapsto \quad c x_{n}, \quad x_{j} \mapsto x_{j}, \quad \bar{x}_{j} \mapsto \bar{x}_{j} \quad(j \neq n), \\
& \gamma_{0}(v(x))=\frac{x_{0}^{2}}{l^{2} x_{1} \bar{x}_{1}}, \quad \gamma_{1}(v(x))=\frac{\left(l x_{1} \bar{x}_{1}\right)^{2}}{x_{0}^{2} x_{2} \bar{x}_{2}}, \\
& \gamma_{i}(v(x))=\frac{\left(x_{i} \bar{x}_{i}\right)^{2}}{x_{i-1} \bar{x}_{i-1} x_{i+1} \bar{x}_{i+1}}(2 \leq i \leq n-2), \\
& \gamma_{n-1}(v(x))=\frac{\left(x_{n-1} \bar{x}_{n-1}\right)^{2}}{x_{n-2} \bar{x}_{n-2} x_{n}^{2}}, \quad \gamma_{n}(v(x))=\frac{x_{n}^{2}}{x_{n-1} \bar{x}_{n-1}}, \\
& \varepsilon_{0}(v(x))=\frac{x_{0}^{2}+l^{2} x_{1} \bar{x}_{1}}{x_{0}^{3}} .
\end{aligned}
$$

Let us check the condition (5.7) in Lemma 5.3. The following are useful for this purpose:

$$
\begin{aligned}
& y_{i} \bar{y}_{i}=a(x)^{2} x_{n-i} \bar{x}_{n-i}, \quad y_{0}=a(x) x_{n}, \\
& a(v(y))=a(\bar{\sigma}(v(x)))=\frac{1}{a(v(x))} .
\end{aligned}
$$

Using these we can easily check the two conditions $\gamma_{i}=\gamma_{\sigma(i)} \circ \bar{\sigma}$ and $\varepsilon_{i}=\varepsilon_{\sigma(i)} \circ \bar{\sigma}$. The condition $e_{\sigma(i)}^{c}=\bar{\sigma} \circ e_{i}^{c} \circ \bar{\sigma}^{-1}$ for $i=2, \cdots, n-2$ is also immediate from (5.27) and (5.28). Next let us see the case $i=1, n-1$. We have

$$
a\left(e_{n-1}^{c}(v(y))\right)=\frac{y_{n}^{2}+c y_{n-1} \bar{y}_{n-1}}{y_{n}^{2} y_{n-1} \frac{c y_{n-1} \bar{y}_{n-1} y+y_{n}^{2}}{y_{n-1} \bar{y}_{n-1} y+y_{n}^{2}}}=\frac{y_{n-1} \bar{y}_{n-1} y+y_{n}^{2}}{y_{n-1} y_{n}^{2}}=\frac{1}{a(v(x))} .
$$


Using this, we can get $e_{n-i}^{c}=\bar{\sigma} \circ e_{1}^{c} \circ \bar{\sigma}^{-1}$ and then $e_{1}^{c}=\bar{\sigma} \circ e_{n-1}^{c} \circ \bar{\sigma}^{-1}$ since $\bar{\sigma}^{2}=\mathrm{id}$. Now, it remains to show that

$$
e_{0}^{c_{1}} e_{n}^{c_{2}}=e_{n}^{c_{2}} e_{0}^{c_{1}}, \quad \varepsilon_{0}\left(e_{n}^{c}(v(x))\right)=\varepsilon_{0}(v(x)), \quad \varepsilon_{n}\left(e_{0}^{c}(v(x))\right)=\varepsilon_{n}(v(x)) .
$$

They easily follow from the explicit form of $e_{0}^{c}$. Thus, the proof of Theorem 5.2 in this case is completed.

5.8. $A_{2 n}^{(2)}$-case $(n \geq 2)$. As in the beginning of this section, we have

$$
w_{1}=s_{0} s_{1} \cdots s_{n} s_{n-1} \cdots s_{2} s_{1}
$$

and

$$
\begin{aligned}
\mathcal{V}\left(A_{2 n}^{(2)}\right)_{l}:=\left\{v_{1}(x)=Y_{0}\left(x_{0}\right) Y_{1}\left(x_{1}\right)\right. & \cdots Y_{n}\left(x_{n}\right) Y_{n-1}\left(\bar{x}_{n-1}\right) \\
& \left.\cdots Y_{1}\left(\bar{x}_{1}\right) l^{H} v_{1} \mid x_{i}, \bar{x}_{i} \in \mathbb{C}^{\times}\right\} .
\end{aligned}
$$

By the explicit description of $W\left(\varpi_{1}\right)_{l}$ as in 4.9 , on $W\left(\varpi_{1}\right)_{l}$ we have

$$
y_{i}\left(c^{-1}\right)=\exp \left(c^{-1} f_{i}\right)= \begin{cases}1+c^{-1} f_{i} & i \neq 0 \\ 1+c^{-1} f_{0}+\frac{1}{2 c^{2}} f_{0}^{2} & i=0\end{cases}
$$

Then we have

$$
v_{1}(x)=l^{m}\left\{\left(\sum_{i=1}^{n} \xi_{i}(x) v_{i}\right)+x_{0}^{2} v_{\overline{1}}+\left(\sum_{i=2}^{n} x_{i-1} v_{\bar{i}}+\right)+\frac{x_{0}}{l} \emptyset\right\},
$$

where

$$
m:=\varpi_{1}(H), \quad \xi_{i}(x):= \begin{cases}\frac{l^{-2} x_{0}^{2}+x_{1} \bar{x}_{1}}{x_{0}^{2}} & i=1, \\ \frac{x_{i-1} \bar{x}_{i-1}+x_{i} \bar{x}_{i}}{x_{i-1}} & i \neq 1, n, \\ \frac{x_{n-1} \bar{x}_{n-1}+x_{n}}{x_{n-1}} & i=n .\end{cases}
$$

Next, for $w_{2}=s_{n} s_{n-1} \cdots s_{1} s_{0} s_{1} \cdots s_{n-1}$, we set

$$
\begin{aligned}
\mathcal{V}_{2}\left(A_{2 n}^{(2)}\right)_{l}:=\left\{v_{2}(y)=Y_{n}\left(y_{n}\right) \cdots\right. & Y_{1}\left(y_{1}\right) Y_{0}\left(y_{0}\right) Y_{1}\left(\bar{y}_{1}\right) \cdots \\
& \left.Y_{n-1}\left(\bar{y}_{n-1}\right) l^{H^{\prime}} v_{\bar{n}} \mid y_{i}, \bar{y}_{i} \in \mathbb{C}^{\times}\right\} .
\end{aligned}
$$

Then we have

$$
v_{2}(y)=l^{m}\left\{\left(\sum_{i=1}^{n} \frac{y_{i}}{l^{2}} v_{i}\right)+\left(\sum_{i=1}^{n} \eta_{i}(y) v_{\bar{i}}\right)+\frac{y_{0}}{l} \emptyset\right\}
$$

where

$$
\eta_{i}(y):= \begin{cases}\frac{y_{0}^{2}+y_{1} \bar{y}_{1}}{y_{1}} & i=1, \\ \frac{y_{i-1} \bar{y}_{i-1}+y_{i} \bar{y}_{i}}{y_{i}} & i \neq 1, n, \\ \frac{y_{n-1} \bar{y}_{n-1}+l^{-2} y_{n}}{y_{n}} & i=n .\end{cases}
$$


Note that $\varpi_{1}(H)=m=\operatorname{wt}\left(v_{\bar{n}}\right)\left(H^{\prime}\right)=\left(\varpi_{n}\left(H^{\prime}\right)\right)$. For $x \in\left(\mathbb{C}^{\times}\right)^{2 n}$ there exist a unique $y=\left(y_{0}, \ldots, \bar{y}_{1}\right) \in\left(\mathbb{C}^{\times}\right)^{2 n}$ and $a(x)$ such that $v_{2}(y)=a(x) v_{1}(x)$. They are given by

$$
\begin{aligned}
& a(x)=\frac{x_{n-1} \bar{x}_{n-1}+x_{n}}{l^{2} x_{n-1} x_{n}}, \\
& y_{0}=a(x) x_{0}=\frac{x_{0}\left(x_{n-1} \bar{x}_{n-1}+x_{n}\right)}{l^{2} x_{n-1} x_{n}}, \\
& y_{1}=l^{2} a(x) \xi_{1}(x)=\frac{\left(x_{n-1} \bar{x}_{n-1}+x_{n}\right)\left(x_{0}+l^{2} x_{1} \bar{x}_{1}\right)}{x_{0}^{2} x_{n-1} x_{n}}, \\
& y_{i}=l^{2} a(x) \xi_{i}(x)=\frac{\left(x_{i-1} \bar{x}_{i-1}+x_{i} \bar{x}_{i}\right)\left(x_{n-1} \bar{x}_{n-1}+x_{n}\right)}{x_{i-1} x_{n-1} x_{n}} \quad(1<i<n), \\
& y_{n}=l^{2} a(x) \xi_{n}=\frac{\left(x_{n-1} \bar{x}_{n-1}+x_{n}\right)^{2}}{x_{n-1}^{2} x_{n}}, \\
& \bar{y}_{1}=a(x) \frac{l^{2} x_{0}^{2} x_{1} \bar{x}_{1}}{x_{0}+l^{2} x_{1} \bar{x}_{1}}=\frac{\left(x_{n-1} \bar{x}_{n-1}+x_{n}\right) x_{0}^{2} x_{1} \bar{x}_{1}}{\left(x_{0}+l^{2} x_{1} \bar{x}_{1}\right) x_{n-1} x_{n}}, \\
& \bar{y}_{i}=a(x) \frac{x_{i-1} x_{i} \bar{x}_{i}}{x_{i-1} \bar{x}_{i-1}+x_{i} \bar{x}_{i}}=\frac{\left(x_{n-1} \bar{x}_{n-1}+x_{n}^{2}\right) x_{i-1} x_{i} \bar{x}_{i}}{l^{2}\left(x_{i-1} \bar{x}_{i-1}+x_{i} \bar{x}_{i}\right) x_{n-1} x_{n}^{2}} \quad(1<i \leq n-1) .
\end{aligned}
$$

It defines a rational mapping $\bar{\sigma}: \mathcal{V}\left(A_{2 n}^{(2)}\right)_{l} \longrightarrow \mathcal{V}_{2}\left(A_{2 n}^{(2)}\right)_{l}\left(v_{1}(x) \mapsto v_{2}(y)\right)$.

The inverse $\bar{\sigma}^{-1}: \mathcal{V}_{2}\left(A_{2 n}^{(2)}\right)_{l} \longrightarrow \mathcal{V}\left(A_{2 n}^{(2)}\right)_{l}\left(v_{2}(y) \mapsto v_{1}(x)\right)$ is given by

$$
\begin{aligned}
& a(y):=\frac{y_{0}^{2} y_{1}}{y_{0}^{2}+y_{1} \bar{y}_{1}}(=a(x)), \\
& x_{0}=\frac{y_{0}}{a(y)}, \\
& x_{i}=a(y)^{-1} \frac{y_{i} \bar{y}_{i}+y_{i+1} \bar{y}_{i+1}}{y_{i+1}} \quad(1 \leq i \leq n-2), \\
& x_{n-1}=a(y)^{-1} \frac{y_{n-1} \bar{y}_{n-1}+l^{-2} y_{n}}{y_{n}}, \\
& x_{n}=\frac{y_{n}}{l^{4} a(y)^{2}}, \quad(1 \leq i \leq n-2), \\
& \bar{x}_{i}=\left(l^{2} a(y)\right)^{-1} \frac{y_{i} \bar{y}_{i} y_{i+1}}{y_{i} \bar{y}_{i}+y_{i+1} \bar{y}_{i+1}} \quad \\
& \bar{x}_{n-1}=\frac{y_{n-1} \bar{y}_{n-1} y_{n}}{a(y)\left(l^{2} y_{n-1} \bar{y}_{n-1}+y_{n}\right)},
\end{aligned}
$$

which means that the morphism $\bar{\sigma}: \mathcal{V}\left(A_{2 n}^{(2)}\right)_{l} \longrightarrow \mathcal{V}_{2}\left(A_{2 n}^{(2)}\right)_{l}$ is birational. Thus, we obtain Theorem [5.1(ii).

The actions of $e_{i}(0 \leq i<n)$ on $v_{2}(y)$ are induced from the ones on $Y_{\mathbf{i}_{2}}(y)$. $l^{H^{\prime}}:=Y_{n}\left(y_{n}\right) \cdots Y_{n-1}\left(\bar{y}_{n-1}\right) \cdot l^{H^{\prime}}\left(\mathbf{i}_{2}=(n, \ldots, 1,0,1, \cdots, n-1)\right)$ since $e_{i} v_{\bar{n}}=0$ for $i=0,1, \ldots, n-1$. We also get $\gamma_{i}\left(v_{2}(y)\right)$ and $\varepsilon_{i}\left(v_{2}(y)\right)$ from the ones for $Y_{\mathbf{i}_{\mathbf{2}}}(y) \cdot l^{H^{\prime}}$ 
where $v_{2}(y)=\bar{\sigma}\left(v_{1}(x)\right)$ :

$$
\begin{aligned}
& e_{0}^{c}: y_{0} \mapsto c y_{0}, \quad y_{i} \mapsto y_{i}, \quad \bar{y}_{i} \mapsto \bar{y}_{i}(i \neq 0), \\
& e_{1}^{c}: y_{1} \mapsto y_{1} \frac{c y_{1} \bar{y}_{1}+y_{0}^{2}}{y_{1} \bar{y}_{1}+y_{0}^{2}}, \quad \bar{y}_{1} \mapsto \bar{y}_{1} \frac{c\left(y_{1} \bar{y}_{1}+y_{0}^{2}\right)}{c y_{1} \bar{y}_{1}+y_{0}^{2}}, \quad y_{i} \mapsto y_{i}, \quad \bar{y}_{i} \mapsto \bar{y}_{i}(i \neq 1), \\
& e_{i}^{c}: y_{i} \mapsto y_{i} \frac{c y_{i} \bar{y}_{i}+y_{i-1} \bar{y}_{i-1}}{y_{i} \bar{y}_{i}+y_{i-1} \bar{y}_{i-1}}, \quad \bar{y}_{i} \mapsto \bar{y}_{i} \frac{c\left(y_{i} \bar{y}_{i}+y_{i-1} \bar{y}_{i-1}\right)}{c y_{i} \bar{y}_{i}+y_{i-1} \bar{y}_{i-1}}, \\
& \quad y_{j} \mapsto y_{j}, \quad \bar{y}_{j} \mapsto \bar{y}_{j}(j \neq i), \quad(i=2, \ldots, n-1), \\
& \gamma_{0}\left(v_{2}(y)\right)=\frac{y_{0}^{2}}{y_{1} \bar{y}_{1}}, \quad \gamma_{1}\left(v_{2}(y)\right)=\frac{\left(y_{1} \bar{y}_{1}\right)^{2}}{y_{0}^{2} y_{2} \bar{y}_{2}}, \\
& \gamma_{i}\left(v_{2}(y)\right)=\frac{\left(y_{i} \bar{y}_{i}\right)^{2}}{y_{i-1} \bar{y}_{i-1} y_{i+1} \bar{y}_{i+1}} \quad(i=2, \ldots, n-2), \\
& \gamma_{n-1}\left(v_{2}(y)\right)=\frac{\left(y_{n-1} \bar{y}_{n-1}\right)^{2}}{y_{n-2} \bar{y}_{n-2} y_{n}}, \quad y_{0}^{2}\left(v_{2}(y)\right)=\frac{y_{1}}{y_{0}}, \varepsilon_{1}\left(v_{2}(y)\right)=\frac{y_{2}}{y_{1}}\left(1+\frac{y_{0}^{2}}{y_{1} \bar{y}_{1}}\right) \\
& \varepsilon_{n-1}\left(v_{2}(y)\right)=\frac{y_{n}}{y_{n-1}}\left(1+\frac{y_{n-2} \bar{y}_{n-2}}{y_{n-1} \bar{y}_{n-1}}\right) \\
& \varepsilon_{i}\left(v_{2}(y)\right)=\frac{y_{i+1}}{y_{i}}\left(1+\frac{y_{i-1} \bar{y}_{i-1}}{y_{i} \bar{y}_{i}}\right) \quad(i=2 \cdots, n-1) .
\end{aligned}
$$

The explicit forms of $\varepsilon_{i}\left(v_{1}(x)\right)$ and $\gamma_{i}\left(v_{1}(x)\right)(1 \leq i \leq n)$ are also induced from the ones for $Y_{\mathbf{i}_{1}}(x) \cdot l^{H}:=Y_{0}\left(x_{0}\right) \cdots Y_{1}\left(\bar{x}_{1}\right) \cdot l^{H}$ and, we define $\varepsilon_{0}\left(v_{1}(x)\right):=\varepsilon_{0}\left(v_{2}(y)\right)$ and $\gamma_{0}\left(v_{1}(x)\right):=\gamma_{0}\left(v_{2}(y)\right)\left(v_{2}(y):=\bar{\sigma}\left(v_{1}(x)\right)\right)$ :

$$
\begin{aligned}
\varepsilon_{0}\left(v_{1}(x)\right) & =\frac{1}{x_{0}}\left(1+\frac{l^{2} x_{1} \bar{x}_{1}}{x_{0}^{2}}\right)^{2}, \\
\varepsilon_{i}\left(v_{1}(x)\right) & =\frac{x_{i-1}}{x_{i}}\left(1+\frac{x_{i+1} \bar{x}_{i+1}}{x_{i} \bar{x}_{i}}\right) \quad(1<i \leq n-2), \\
\varepsilon_{1}\left(v_{1}(x)\right) & =\frac{x_{0}^{2}}{x_{1}}\left(1+\frac{x_{2} \bar{x}_{2}}{x_{1} \bar{x}_{1}}\right), \\
\varepsilon_{n-1}\left(v_{1}(x)\right) & =\frac{x_{n-2}}{x_{n-1}}\left(1+\frac{x_{n}}{x_{n-1} \bar{x}_{n-1}}\right), \quad \varepsilon_{n}\left(v_{1}(x)\right)=\frac{x_{n-1}^{2}}{x_{n}} . \\
\gamma_{0}\left(v_{1}(x)\right) & =\frac{x_{0}^{2}}{l^{4} x_{1} \bar{x}_{1}}, \gamma_{1}\left(v_{1}(x)\right)=\frac{\left(l x_{1} \bar{x}_{1}\right)^{2}}{x_{0}^{2} x_{2} \bar{x}_{2}}, \\
\gamma_{i}\left(v_{1}(x)\right) & =\frac{\left(x_{i} \bar{x}_{i}\right)^{2}}{x_{i-1} \bar{x}_{i-1} x_{i+1} \bar{x}_{i+1}}(2 \leq i \leq n-2), \\
\gamma_{n-1}\left(v_{1}(x)\right) & =\frac{\left(x_{n-1} \bar{x}_{n-1}\right)^{2}}{x_{n-2} \bar{x}_{n-2} x_{n}}, \quad \gamma_{n}\left(v_{1}(x)\right)=\frac{x_{n}^{2}}{\left(x_{n-1} \bar{x}_{n-1}\right)^{2}} .
\end{aligned}
$$


For $i=0$, we define $e_{0}^{c}\left(v_{1}(x)\right)=\bar{\sigma}^{-1} \circ e_{0}^{c} \circ \bar{\sigma}\left(v_{1}(x)\right)=\bar{\sigma}^{-1} \circ e_{0}^{c}\left(v_{2}(y)\right)$. Then we get

$$
\begin{array}{cccc}
e_{0}^{c}: & x_{0} \mapsto \quad x_{0} \frac{c^{2} x_{0}+l^{2} x_{1} \bar{x}_{1}}{c\left(x_{0}^{2}+l^{2} x_{1} \bar{x}_{1}\right)}, \quad x_{i} \mapsto x_{i} \frac{c^{2} x_{0}+l^{2} x_{1} \bar{x}_{1}}{c^{2}\left(x_{0}^{2}+l^{2} x_{1} \bar{x}_{1}\right)}(1 \leq i<n), \\
& \bar{x}_{i} \mapsto \quad \bar{x}_{i} \frac{c^{2} x_{0}+l^{2} x_{1} \bar{x}_{1}}{c^{2}\left(x_{0}^{2}+l^{2} x_{1} \bar{x}_{1}\right)}(1 \leq i \leq n-1), x_{n} \mapsto x_{n} \frac{\left(c^{2} x_{0}+l^{2} x_{1} \bar{x}_{1}\right)^{2}}{c^{4}\left(x_{0}^{2}+l^{2} x_{1} \bar{x}_{1}\right)^{2}}, \\
e_{i}^{c}: & x_{i} \mapsto \quad x_{i} \frac{c x_{i} \bar{x}_{i}+x_{i+1} \bar{x}_{i+1}}{x_{i} \bar{x}_{i}+x_{i+1} \bar{x}_{i+1}}, \quad \bar{x}_{i} \mapsto \bar{x}_{i} \frac{c\left(x_{i} \bar{x}_{i}+x_{i+1} \bar{x}_{i+1}\right)}{c x_{i} \bar{x}_{i}+x_{i+1} \bar{x}_{i+1}}, \\
x_{j-1}: \quad x_{n-1} \mapsto \quad \bar{x}_{j} \mapsto \bar{x}_{j}(j \neq i) \quad(1 \leq i<n-1) & x_{n-1} \frac{c x_{n-1} \bar{x}_{n-1}+x_{n}}{x_{n-1} \bar{x}_{n-1}+x_{n}}, \quad \bar{x}_{n-1} \mapsto \bar{x}_{n-1} \frac{c\left(x_{n-1} \bar{x}_{n-1}+x_{n}\right)}{c x_{n-1} \bar{x}_{n-1}+x_{n}}, \\
& & x_{i} \mapsto x_{i}, \quad \bar{x}_{i} \mapsto \bar{x}_{i}(i \neq n-1), \\
e_{n}^{c}: & x_{n} \mapsto \quad c x_{n}, \quad x_{i} \mapsto x_{i}, \quad \bar{x}_{i} \mapsto \bar{x}_{i}(i \neq n) .
\end{array}
$$

In order to prove Theorem 5.2 it suffices to show the following:

$$
\begin{aligned}
& e_{i}^{c}=\bar{\sigma}^{-1} \circ e_{i}^{c} \circ \bar{\sigma}, \gamma_{i}=\gamma_{i} \circ \bar{\sigma}, \varepsilon_{i}=\varepsilon_{i} \circ \bar{\sigma}(i \neq 0, n), \\
& e_{0}^{c_{1}} e_{n}^{c_{2}}=e_{n}^{c_{2}} e_{0}^{c_{1}}, \\
& \gamma_{n}\left(e_{0}^{c}\left(v_{1}(x)\right)\right)=\gamma_{n}\left(v_{1}(x)\right), \quad \gamma_{0}\left(e_{n}^{c}\left(v_{1}(x)\right)\right)=\gamma_{0}\left(v_{1}(x)\right), \\
& \varepsilon_{0}\left(e_{0}^{c}\left(v_{1}(x)\right)\right)=c^{-1} \varepsilon_{0}\left(v_{1}(x)\right),
\end{aligned}
$$

which are immediate from the above formulae. Let us show (5.29). Set $v_{2}(y):=$ $\bar{\sigma}\left(v_{1}(x)\right)$ and $v_{1}\left(x^{\prime}\right):=\bar{\sigma}^{-1}\left(e_{i}^{c}\left(v_{2}(y)\right)\right)$ for $i=2, \ldots, n-2$. Then $x_{j}^{\prime}=x_{j}$ and $\bar{x}_{j}^{\prime}=\bar{x}_{j}$ for $j \neq i-1, i$, and we have

$$
\begin{aligned}
& a\left(v_{2}(y)\right)=a\left(v_{1}(x)\right), \\
& x_{i}^{\prime}=\frac{1}{a\left(v_{2}(y)\right)}\left(\bar{y}_{i+1}+\frac{c y_{i} \bar{y}_{i}}{y_{i+1}}\right)=x_{i} \frac{c x_{i} \bar{x}_{i}+x_{i+1} \bar{x}_{i+1}}{x_{i} \bar{x}_{i}+x_{i+1} \bar{x}_{i+1}}, \\
& \bar{x}_{i}^{\prime}=\frac{1}{a\left(v_{2}(y)\right)} \frac{c y_{i} \bar{y}_{i} y_{i+1}}{c y_{i} \bar{y}_{i}+y_{i+1} \bar{y}_{i+1}}=\bar{x}_{i} \frac{c\left(x_{i} \bar{x}_{i}+x_{i+1} \bar{x}_{i+1}\right)}{c x_{i} \bar{x}_{i}+x_{i+1} \bar{x}_{i+1}}, \\
& x_{i-1}^{\prime}=\frac{1}{a\left(v_{2}(y)\right)}\left(\bar{y}_{i}+\frac{c y_{i-1} \bar{y}_{i-1}}{y_{i}}\right)=x_{i-1}, \\
& \bar{x}_{i-1}^{\prime}=\frac{1}{a\left(v_{2}(y)\right)} \frac{c y_{i-1} \bar{y}_{i-1} y_{i}}{c y_{i} \bar{y}_{i}+y_{i-1} \bar{y}_{i-1}}=\bar{x}_{i-1},
\end{aligned}
$$

where the formula $y_{i} \bar{y}_{i}=a\left(v_{1}(x)\right) x_{i} \bar{x}_{i}$ is useful to obtain these results. Therefore we have $e_{i}^{c}=\bar{\sigma}^{-1} \circ e_{i}^{c} \circ \bar{\sigma}$ for $i=2, \ldots, n-2$. Others are obtained similarly.

5.9. $A_{2 n}^{(2) \dagger}$-case $(n \geq 2)$. As in the beginning of this section, we have

$$
w_{1}=s_{0} s_{1} \cdots s_{n} s_{n-1} \cdots s_{2} s_{1}
$$

and

$$
\begin{aligned}
\mathcal{V}\left(A_{2 n}^{(2) \dagger}\right)_{l}:=\left\{v_{1}(x)=Y_{0}\left(x_{0}\right) Y_{1}\left(x_{1}\right) \cdots\right. & Y_{n}\left(x_{n}\right) Y_{n-1}\left(\bar{x}_{n-1}\right) \cdots \\
& \left.Y_{1}\left(\bar{x}_{1}\right) l^{H} v_{1} \mid x_{i}, \bar{x}_{i} \in \mathbb{C}^{\times}\right\} .
\end{aligned}
$$

By the explicit description of $W\left(\varpi_{1}\right)_{l}$ as in 4.9 , on $W\left(\varpi_{1}\right)_{l}$ we have

$$
y_{i}\left(c^{-1}\right)=\exp \left(c^{-1} f_{i}\right)= \begin{cases}1+c^{-1} f_{i} & i \neq n, \\ 1+c^{-1} f_{n}+\frac{1}{2 c^{2}} f_{n}^{2} & i=n .\end{cases}
$$


Then we have

$$
v_{1}(x)=l^{m}\left\{\left(\sum_{i=1}^{n} \xi_{i}(x) v_{i}\right)+x_{n} v_{0}+\left(\sum_{i=1}^{n} x_{i-1} v_{\bar{i}}\right)\right\}
$$

where

$$
\xi_{i}(x):= \begin{cases}\frac{l^{-1} x_{0}+x_{1} \bar{x}_{1}}{x_{0}} & i=1, \\ \frac{x_{i-1} \bar{x}_{i-1}+x_{i} \bar{x}_{i}}{x_{i-1}} & i \neq 1, n, \\ \frac{x_{n-1} \bar{x}_{n-1}+x_{n}^{2}}{x_{n-1}} & i=n,\end{cases}
$$

and $m:=\varpi_{1}(H)$. Next, for $w_{2}=s_{n} s_{n-1} \cdots s_{1} s_{0} s_{1} \cdots s_{n-1}$ and $H^{\prime}$ such that $m=\varpi_{n}\left(H^{\prime}\right)$, we set

$$
\begin{aligned}
\mathcal{V}_{2}\left(A_{2 n}^{(2) \dagger}\right)_{l}:=\left\{v_{2}(y)=Y_{n}\left(y_{n}\right) \cdots\right. & Y_{1}\left(y_{1}\right) Y_{0}\left(y_{0}\right) Y_{1}\left(\bar{y}_{1}\right) \cdots \\
& \left.Y_{n-1}\left(\bar{y}_{n-1}\right) l^{H^{\prime}} v_{\bar{n}} \mid y_{i}, \bar{y}_{i} \in \mathbb{C}^{\times}\right\} .
\end{aligned}
$$

Then we have

$$
v_{2}(y)=l^{m}\left\{\left(\sum_{i=1}^{n-1} \frac{y_{i}}{l} v_{i}\right)+\frac{y_{n}^{2}}{l} v_{n}+\frac{y_{n}}{l} v_{0}+\left(\sum_{i=1}^{n} \eta_{i}(y) v_{\bar{i}}\right)\right\}
$$

where

$$
\eta_{i}(y):= \begin{cases}\frac{y_{0}+y_{1} \bar{y}_{1}}{y_{1}} & i=1, \\ \frac{y_{i-1} \bar{y}_{i-1}+y_{i} \bar{y}_{i}}{y_{i}} & i \neq 1, n, \\ \frac{y_{n-1} \bar{y}_{n-1}+l^{-1} y_{n}^{2}}{y_{n}^{2}} & i=n .\end{cases}
$$

For $x \in\left(\mathbb{C}^{\times}\right)^{2 n}$ there exist a unique $y=\left(y_{0}, \ldots, \bar{y}_{1}\right) \in\left(\mathbb{C}^{\times}\right)^{2 n}$ and $a(x)$ such that $v_{2}(y)=a(x) v_{1}(x)$. They are given by

$$
\begin{aligned}
& a(x)=\frac{x_{n-1} \bar{x}_{n-1}+x_{n}^{2}}{l x_{n-1} x_{n}^{2}}, \\
& y_{0}=a(x)^{2} x_{0}=\frac{x_{0}\left(x_{n-1} \bar{x}_{n-1}+x_{n}^{2}\right)^{2}}{\left(l x_{n-1} x_{n}^{2}\right)^{2}}, \\
& y_{1}=l a(x) \xi_{1}(x)=\frac{\left(x_{n-1} \bar{x}_{n-1}+x_{n}^{2}\right)\left(l^{-1} x_{0}+x_{1} \bar{x}_{1}\right)}{x_{0} x_{n-1} x_{n}^{2}}, \\
& y_{i}=l a(x) \xi_{i}(x)=\frac{\left(x_{i-1} \bar{x}_{i-1}+x_{i} \bar{x}_{i}\right)\left(x_{n-1} \bar{x}_{n-1}+x_{n}^{2}\right)}{x_{i-1} x_{n-1} x_{n}^{2}} \quad(1<i<n), \\
& y_{n}=l a(x) x_{n}=\frac{x_{n-1} \bar{x}_{n-1}+x_{n}^{2}}{x_{n-1} x_{n}}, \quad(1<i \leq n-1) . \\
& \bar{y}_{1}=a(x) \frac{x_{0} x_{1} \bar{x}_{1}}{l^{-1} x_{0}+x_{1} \bar{x}_{1}}=\frac{\left(x_{n-1} \bar{x}_{n-1}+x_{n}^{2}\right) x_{0} x_{1} \bar{x}_{1}}{\left(x_{0}+l x_{1} \bar{x}_{1}\right) x_{n-1} x_{n}^{2}}, \\
& \bar{y}_{i}=a(x) \frac{x_{i-1} x_{i} \bar{x}_{i}}{x_{i-1} \bar{x}_{i-1}+x_{i} \bar{x}_{i}}=\frac{\left(x_{n-1} \bar{x}_{n-1}+x_{n}^{2}\right) x_{i-1} x_{i} \bar{x}_{i}}{l\left(x_{i-1} \bar{x}_{i-1}+x_{i} \bar{x}_{i}\right) x_{n-1} x_{n}^{2}} \quad(1<1<
\end{aligned}
$$


It defines a rational mapping $\bar{\sigma}: \mathcal{V}\left(A_{2 n}^{(2) \dagger}\right) \longrightarrow \mathcal{V}_{2}\left(A_{2 n}^{(2) \dagger}\right)\left(v_{1}(x) \mapsto v_{2}(y)\right)$.

The inverse $\bar{\sigma}^{-1}: \mathcal{V}_{2}\left(A_{2 n}^{(2) \dagger}\right) \longrightarrow \mathcal{V}\left(A_{2 n}^{(2) \dagger}\right)\left(v_{2}(y) \mapsto v_{1}(x)\right)$ is given by

$$
\begin{aligned}
& a(y):=\frac{y_{0} y_{1}}{y_{0}+y_{1} \bar{y}_{1}}(=a(x)), \\
& x_{0}=a(y)^{-1} \frac{y_{0}+y_{1} \bar{y}_{1}}{y_{1}}, \\
& x_{i}=a(y)^{-1} \frac{y_{i} \bar{y}_{i}+y_{i+1} \bar{y}_{i+1}}{y_{i+1}} \quad(1 \leq i \leq n-2), \\
& x_{n-1}=a(y)^{-1} \frac{y_{n-1} \bar{y}_{n-1}+l^{-1} y_{n}^{2}}{y_{n}^{2}}, \\
& x_{n}=\frac{y_{n}}{l a(y)}, \\
& \bar{x}_{i}=(l a(y))^{-1} \frac{y_{i} \bar{y}_{i} y_{i+1}}{y_{i} \bar{y}_{i}+y_{i+1} \bar{y}_{i+1}} \quad(1 \leq i \leq n-2), \\
& \bar{x}_{n-1}=a(y)^{-1} \frac{y_{n-1} \bar{y}_{n-1} y_{n}^{2}}{l y_{n-1} \bar{y}_{n-1}+y_{n}^{2}},
\end{aligned}
$$

which means that the morphism $\bar{\sigma}: \mathcal{V}\left(A_{2 n}^{(2) \dagger}\right) \longrightarrow \mathcal{V}_{2}\left(A_{2 n}^{(2) \dagger}\right)$ is birational. Thus, we obtain Theorem 5.1(ii).

The actions of $e_{i}(0 \leq i<n)$ on $v_{2}(y)$ are induced from the ones on $Y_{\mathbf{i}_{2}}(y)$. $l^{H^{\prime}}:=Y_{n}\left(y_{n}\right) \cdots Y_{n-1}\left(\bar{y}_{n-1}\right) \cdot l^{H^{\prime}}\left(\mathbf{i}_{2}=(n, \ldots, 1,0,1, \ldots, n-1)\right)$ since $e_{i} v_{\bar{n}}=0$ for $i=0,1, \ldots, n-1$. We also get $\gamma_{i}\left(v_{2}(y)\right)$ and $\varepsilon_{i}\left(v_{2}(y)\right)$ from the ones for $Y_{\mathbf{i}_{\mathbf{2}}}(y) \cdot l^{H^{\prime}}$ where $v_{2}(y)=\bar{\sigma}\left(v_{1}(x)\right)$ :

$$
\begin{aligned}
& e_{0}^{c}: y_{0} \mapsto c y_{0}, \quad y_{i} \mapsto y_{i}, \quad \bar{y}_{i} \mapsto \bar{y}_{i}(i \neq 0), \\
& e_{1}^{c}: y_{1} \mapsto y_{1} \frac{c y_{1} \bar{y}_{1}+y_{0}}{y_{1} \bar{y}_{1}+y_{0}}, \quad \bar{y}_{1} \mapsto \bar{y}_{1} \frac{c\left(y_{1} \bar{y}_{1}+y_{0}\right)}{c y_{1} \bar{y}_{1}+y_{0}}, \quad y_{i} \mapsto y_{i}, \quad \bar{y}_{i} \mapsto \bar{y}_{i}(i \neq 1), \\
& e_{i}^{c}: y_{i} \mapsto y_{i} \frac{c y_{i} \bar{y}_{i}+y_{i-1} \bar{y}_{i-1}}{y_{i} \bar{y}_{i}+y_{i-1} \bar{y}_{i-1}}, \quad \bar{y}_{i} \mapsto \bar{y}_{i} \frac{c\left(y_{i} \bar{y}_{i}+y_{i-1} \bar{y}_{i-1}\right)}{c y_{i} \bar{y}_{i}+y_{i-1} \bar{y}_{i-1}}, \\
& \quad y_{j} \mapsto y_{j}, \quad \bar{y}_{j} \mapsto \bar{y}_{j}(j \neq i), \quad(i=2, \ldots, n-1), \\
& \gamma_{0}\left(v_{2}(y)\right)=\frac{y_{0}^{2}}{\left(y_{1} \bar{y}_{1}\right)^{2}}, \quad \gamma_{1}\left(v_{2}(y)\right)=\frac{\left(y_{1} \bar{y}_{1}\right)^{2}}{y_{0} y_{2} \bar{y}_{2}}, \\
& \gamma_{i}\left(v_{2}(y)\right)=\frac{\left(y_{i} \bar{y}_{i}\right)^{2}}{y_{i-1} \bar{y}_{i-1} y_{i+1} \bar{y}_{i+1}} \quad(i=2, \ldots, n-2), \\
& \left.\gamma_{n-1}\left(v_{2}(y)\right)=\frac{\left(y_{n-1} \bar{y}_{n-1}\right)^{2}}{y_{n-2} \bar{y}_{n-2} y_{n}^{2}}, \quad v_{2}(y)\right)=\frac{y_{1}^{2}}{y_{0}}, \varepsilon_{1}\left(v_{2}(y)\right)=\frac{y_{2}}{y_{1}}\left(1+\frac{y_{0}}{y_{1} \bar{y}_{1}}\right) \\
& \varepsilon_{n-1}\left(v_{2}(y)\right)=\frac{y_{n}^{2}}{y_{n-1}}\left(1+\frac{y_{n-2} \bar{y}_{n-2}}{y_{n-1} \bar{y}_{n-1}}\right) \\
& \varepsilon_{i}\left(v_{2}(y)\right)=\frac{y_{i+1}}{y_{i}}\left(1+\frac{y_{i-1} \bar{y}_{i-1}}{y_{i} \bar{y}_{i}}\right) \quad(i=2 \cdots, n-1) .
\end{aligned}
$$


The explicit forms of $\varepsilon_{i}\left(v_{1}(x)\right)$ and $\gamma_{i}\left(v_{1}(x)\right)(1 \leq i \leq n)$ are also induced from the ones for $Y_{\mathbf{i}_{1}}(x) \cdot l^{H}:=Y_{0}\left(x_{0}\right) \cdots Y_{1}\left(\bar{x}_{1}\right) \cdot l^{H}$ and, we define $\varepsilon_{0}\left(v_{1}(x)\right):=\varepsilon_{0}\left(v_{2}(y)\right)$ and $\gamma_{0}\left(v_{1}(x)\right):=\gamma_{0}\left(v_{2}(y)\right)\left(v_{2}(y):=\bar{\sigma}\left(v_{1}(x)\right)\right)$ :

$$
\begin{aligned}
\varepsilon_{0}\left(v_{1}(x)\right) & =\frac{1}{x_{0}}\left(1+\frac{l x_{1} \bar{x}_{1}}{x_{0}}\right)^{2}, \\
\varepsilon_{i}\left(v_{1}(x)\right) & =\frac{x_{i-1}}{x_{i}}\left(1+\frac{x_{i+1} \bar{x}_{i+1}}{x_{i} \bar{x}_{i}}\right) \quad(1 \leq i \leq n-2), \\
\varepsilon_{n-1}\left(v_{1}(x)\right) & =\frac{x_{n-2}}{x_{n-1}}\left(1+\frac{x_{n}^{2}}{x_{n-1} \bar{x}_{n-1}}\right), \quad \varepsilon_{n}\left(v_{1}(x)\right)=\frac{x_{n-1}}{x_{n}} . \\
\gamma_{0}\left(v_{1}(x)\right) & =\frac{x_{0}^{2}}{\left(l x_{1} \bar{x}_{1}\right)^{2}}, \gamma_{1}\left(v_{1}(x)\right)=\frac{\left(l x_{1} \bar{x}_{1}\right)^{2}}{x_{0} x_{2} \bar{x}_{2}}, \\
\gamma_{i}\left(v_{1}(x)\right) & =\frac{\left(x_{i} \bar{x}_{i}\right)^{2}}{x_{i-1} \bar{x}_{i-1} x_{i+1} \bar{x}_{i+1}}(2 \leq i \leq n-2), \\
\gamma_{n-1}\left(v_{1}(x)\right) & =\frac{\left(x_{n-1} \bar{x}_{n-1}\right)^{2}}{x_{n-2} \bar{x}_{n-2} x_{n}^{2}}, \quad \gamma_{n}\left(v_{1}(x)\right)=\frac{x_{n}^{2}}{x_{n-1} \bar{x}_{n-1}} .
\end{aligned}
$$

For $i=0$, we define $e_{0}^{c}\left(v_{1}(x)\right)=\bar{\sigma}^{-1} \circ e_{0}^{c} \circ \bar{\sigma}\left(v_{1}(x)\right)=\bar{\sigma}^{-1} \circ e_{0}^{c}\left(v_{2}(y)\right)$. Then we get

$$
\begin{array}{rcc}
e_{0}^{c}: & x_{0} \mapsto \quad x_{0} \frac{\left(c x_{0}+l x_{1} \bar{x}_{1}\right)^{2}}{c\left(x_{0}+l x_{1} \bar{x}_{1}\right)^{2}}, \quad x_{i} \mapsto x_{i} \frac{c x_{0}+l x_{1} \bar{x}_{1}}{c\left(x_{0}+l x_{1} \bar{x}_{1}\right)}(1 \leq i \leq n), \\
\bar{x}_{i} \mapsto \quad \bar{x}_{i} \frac{c x_{0}+l x_{1} \bar{x}_{1}}{c\left(x_{0}+l x_{1} \bar{x}_{1}\right)}(1 \leq i \leq n-1), \\
e_{i}^{c}: \quad x_{i} \mapsto \quad x_{i} \frac{c x_{i} \bar{x}_{i}+x_{i+1} \bar{x}_{i+1}}{x_{i} \bar{x}_{i}+x_{i+1} \bar{x}_{i+1}}, \quad \bar{x}_{i} \mapsto \bar{x}_{i} \frac{c\left(x_{i} \bar{x}_{i}+x_{i+1} \bar{x}_{i+1}\right)}{c x_{i} \bar{x}_{i}+x_{i+1} \bar{x}_{i+1}}, \\
x_{j} \mapsto x_{j}, \quad \bar{x}_{j} \mapsto \bar{x}_{j}(j \neq i), \quad(1 \leq i<n-1), \\
e_{n-1}^{c}: \quad x_{n-1} \mapsto \quad x_{n-1} \frac{c x_{n-1} \bar{x}_{n-1}+x_{n}^{2}}{x_{n-1} \bar{x}_{n-1}+x_{n}^{2}}, \quad \bar{x}_{n-1} \mapsto \bar{x}_{n-1} \frac{c\left(x_{n-1} \bar{x}_{n-1}+x_{n}^{2}\right)}{c x_{n-1} \bar{x}_{n-1}+x_{n}^{2}}, \\
e_{n}^{c}: \quad x_{n} \mapsto \quad c x_{n}, \quad x_{i} \mapsto x_{i}, \quad \bar{x}_{i} \mapsto \bar{x}_{i}(i \neq n) .
\end{array}
$$

In order to prove Theorem 5.2] it suffices to show the following:

$$
\begin{aligned}
& e_{i}^{c}=\bar{\sigma}^{-1} \circ e_{i}^{c} \circ \bar{\sigma}, \gamma_{i}=\gamma_{i} \circ \bar{\sigma}, \varepsilon_{i}=\varepsilon_{i} \circ \bar{\sigma}(i \neq 0, n), \\
& e_{0}^{c_{1}} e_{n}^{c_{2}}=e_{n}^{c_{2}} e_{0}^{c_{1}}, \\
& \gamma_{n}\left(e_{0}^{c}\left(v_{1}(x)\right)\right)=\gamma_{n}\left(v_{1}(x)\right), \quad \gamma_{0}\left(e_{n}^{c}\left(v_{1}(x)\right)\right)=\gamma_{0}\left(v_{1}(x)\right), \\
& \varepsilon_{0}\left(e_{0}^{c}\left(v_{1}(x)\right)\right)=c^{-1} \varepsilon_{0}\left(v_{1}(x)\right),
\end{aligned}
$$

which are immediate from the above formulae. Let us show (5.33). Set $v_{2}(y):=$ $\bar{\sigma}\left(v_{1}(x)\right)$ and $v_{1}\left(x^{\prime}\right):=\bar{\sigma}^{-1}\left(e_{i}^{c}\left(v_{2}(y)\right)\right)$ for $i=2, \ldots, n-2$. Then $x_{j}^{\prime}=x_{j}$ and $\bar{x}_{j}^{\prime}=\bar{x}_{j}$ 
for $j \neq i-1, i$, and we have

$$
\begin{aligned}
& a\left(v_{2}(y)\right)=a\left(v_{1}(x)\right), \\
& x_{i}^{\prime}=\frac{1}{a\left(v_{2}(y)\right)}\left(\bar{y}_{i+1}+\frac{c y_{i} \bar{y}_{i}}{y_{i+1}}\right)=x_{i} \frac{c x_{i} \bar{x}_{i}+x_{i+1} \bar{x}_{i+1}}{x_{i} \bar{x}_{i}+x_{i+1} \bar{x}_{i+1}}, \\
& \bar{x}_{i}^{\prime}=\frac{1}{a\left(v_{2}(y)\right)} \frac{c y_{i} \bar{y}_{i} y_{i+1}}{c y_{i} \bar{y}_{i}+y_{i+1} \bar{y}_{i+1}}=\bar{x}_{i} \frac{c\left(x_{i} \bar{x}_{i}+x_{i+1} \bar{x}_{i+1}\right)}{c x_{i} \bar{x}_{i}+x_{i+1} \bar{x}_{i+1}}, \\
& x_{i-1}^{\prime}=\frac{1}{a\left(v_{2}(y)\right)}\left(\bar{y}_{i}+\frac{c y_{i-1} \bar{y}_{i-1}}{y_{i}}\right)=x_{i-1}, \\
& \bar{x}_{i-1}^{\prime}=\frac{1}{a\left(v_{2}(y)\right)} \frac{c y_{i-1} \bar{y}_{i-1} y_{i}}{c y_{i} \bar{y}_{i}+y_{i-1} \bar{y}_{i-1}}=\bar{x}_{i-1},
\end{aligned}
$$

where the formula $y_{i} \bar{y}_{i}=a\left(v_{1}(x)\right) x_{i} \bar{x}_{i}$ is useful to obtain these results. Therefore we have $e_{i}^{c}=\bar{\sigma}^{-1} \circ e_{i}^{c} \circ \bar{\sigma}$ for $i=2, \ldots, n-2$. Others are obtained similarly.

5.10. Ultra-discretization of $\mathcal{V}(\mathfrak{g})_{l}$. Let us investigate the ultra-discretization of $\mathcal{V}(\mathfrak{g})_{l}$.

By the explicit forms of the geometric crystal $\mathcal{V}(\mathfrak{g})_{l}$, if we assume that $l$ is a positive real number, it is clear that it has a natural positive structure $\theta_{l}:\left(\mathbb{C}^{\times}\right)^{m} \rightarrow$ $\mathcal{V}(\mathfrak{g})_{l}(x \mapsto v(x))$ where $m=\operatorname{dim} \mathcal{V}(\mathfrak{g})_{l}$. Then we have the following theorem:

Theorem 5.4. For $\mathfrak{g}=A_{n}^{(1)}, B_{n}^{(1)}, D_{n}^{(1)}, D_{n+1}^{(2)}, A_{2 n-1}^{(2)}$ and $A_{2 n}^{(2)}$, suppose that $l>0$. Then the ultra-discretization $\mathcal{U} D_{\theta_{l}}\left(\mathcal{V}(\mathfrak{g})_{l}\right)$ associated with the positive structure $\theta_{l}$ is isomorphic to the crystal $B_{\infty}\left(\mathfrak{g}^{L}\right)$ (4.11).

Proof. First we consider the case $\mathfrak{g} \neq A_{2 n}^{(2)}$. Let $\mathcal{V}(\mathfrak{g})$ be the affine geometric crystal in [KNO]. It follows from its explicit form ([KNO] $)$ that restricting $l=1$, we have the isomorphism

$$
\mathcal{V}(\mathfrak{g})_{l \mid l=1} \stackrel{\sim}{\longrightarrow} \mathcal{V}(\mathfrak{g}) .
$$

The resulting crystal of the ultra-discretization $\mathcal{U} D_{\theta_{l}}$ does not depend on $l$. This and (5.37) imply

$$
\mathcal{U} D_{\theta_{l}}\left(\mathcal{V}(\mathfrak{g})_{l}\right) \cong \mathcal{U} D_{\theta}(\mathcal{V}(\mathfrak{g}))
$$

as crystals. We show in $\mathrm{KNO}$ that $\mathcal{U} D_{\theta}(\mathcal{V}(\mathfrak{g})) \cong B_{\infty}\left(\mathfrak{g}^{L}\right)$. Thus, we have $\mathcal{U} D_{\theta_{l}}\left(\mathcal{V}(\mathfrak{g})_{l}\right) \cong B_{\infty}\left(\mathfrak{g}^{L}\right)$.

As for the case $\mathfrak{g}=A_{2 n}^{(2)}$, we consider as follows: For the geometric crystal $\mathcal{V}\left(A_{2 n}^{(2)^{\dagger}}\right)_{l}$, we define $\bar{e}_{i}:=e_{n-i}, \bar{\gamma}_{i}:=\gamma_{n-i}$ and $\bar{\varepsilon}_{i}:=\varepsilon_{n-i}$ and set $x_{i} \mapsto y_{n-i}(i=$ $0, \ldots, n)$ and $\bar{x}_{i} \mapsto \bar{y}_{n-i}(i=1, \ldots, n-1)$. We denote by $\overline{\mathcal{V}}_{l}$ the $A_{2 n}^{(2)}$-geometric crystal thus obtained. The explicit form of $\overline{\mathcal{V}}_{l}$ is e.g., $\bar{e}_{0}^{c}: y_{0} \mapsto c y_{0}, \bar{\varepsilon}_{0}(y)=\frac{y_{1}}{y_{0}}$ and $\bar{\gamma}_{0}(y)=\frac{y_{n}^{2}}{y_{1} \bar{y}_{1}}$, etc. By using the birational map $\bar{\sigma}$ for $A_{2 n}^{(2)}$ as in 5.8, we obtain the isomorphism of $A_{2 n}^{(2)}$-geometric crystal

$$
\bar{\sigma}: \mathcal{V}\left(A_{2 n}^{(2)}\right)_{l} \stackrel{\sim}{\longrightarrow} \overline{\mathcal{V}}_{l^{2}}
$$

Here $\overline{\mathcal{V}}_{l}$ is isomorphic to $\mathcal{V}\left(A_{2 n}^{(2)^{\dagger}}\right)_{l}$ as an $A_{2 n}^{(2)^{\dagger}}$-geometric crystal. Due to the results in [KNO] and the fact $\left.\mathcal{V}\left(A_{2 n}^{(2)^{\dagger}}\right)_{l}\right|_{l=1}=\mathcal{V}\left(A_{2 n}^{(2)^{\dagger}}\right)$, we have

$$
\mathcal{U} D_{\theta_{l^{2}}}\left(\overline{\mathcal{V}}_{l^{2}}\right) \cong \mathcal{U} D_{\theta_{l^{2}}}\left(\mathcal{V}\left(A_{2 n}^{(2)^{\dagger}}\right)_{l^{2}}\right)=\mathcal{U} D_{\theta}\left(\mathcal{V}\left(A_{2 n}^{(2)^{\dagger}}\right)\right) \cong B_{\infty}\left(A_{2 n}^{(2)}\right) \text {. }
$$


Let us denote by $\bar{B}_{l}$ the perfect crystal $B_{l}\left(A_{2 n}^{(2)}\right)$ considered as an $A_{2 n}^{(2)}{ }^{\dagger}$-perfect crystal. Then the crystal $B_{\infty}\left(A_{2 n}^{(2)}\right)$ can be regarded as the limit of perfect $A_{2 n}^{(2)^{\dagger}}$ crystals $\left\{\bar{B}_{l}\right\}$.

\section{FOLDING OF $D_{n}^{(1)}$-GEOMETRIC CRYSTAL}

6.1. $D_{n}^{(1)}$-geometric crystal $\mathcal{B}_{L}\left(D_{n}^{(1)}\right)$. We review the geometric crystal $\mathcal{B}_{L}\left(D_{n}^{(1)}\right)$ $(n \geq 4)$ in $\mathrm{KOTY}$.

Taking $L \in \mathbb{C}^{\times}$, define the geometric crystal $\mathcal{B}_{L}\left(D_{n}^{(1)}\right):=\left(\mathcal{B}_{L}\left(D_{n}^{(1)}\right),\left\{e_{i}\right\},\left\{\gamma_{i}\right\}\right.$, $\left.\left\{\varepsilon_{i}\right\}\right)$ as follows:

$$
\begin{aligned}
\mathcal{B}_{L}\left(D_{n}^{(1)}\right) & :=\left\{l=\left(l_{1}, \ldots, l_{n}, \bar{l}_{n-1}, \ldots, \bar{l}_{1}\right) \in\left(\mathbb{C}^{\times}\right)^{2 n-1} \mid l_{1} \ldots l_{n} \bar{l}_{n-1} \ldots \bar{l}_{1}=L\right\}, \\
\varepsilon_{0}(l) & =l_{1}\left(\frac{l_{2}}{\bar{l}_{2}}+1\right), \varepsilon_{n-1}(l)=l_{n} \bar{l}_{n-1}, \varepsilon_{n}(l)=\bar{l}_{n-1}, \varepsilon_{i}(l)=\bar{l}_{i}\left(\frac{l_{i+1}}{\bar{l}_{i+1}}+1\right), \\
\gamma_{0}(l) & =\frac{\bar{l}_{1} \bar{l}_{2}}{l_{1} l_{2}}, \gamma_{n-1}(l)=\frac{l_{n-1}}{l_{n} \bar{l}_{n-1}}, \gamma_{n}(l)=\frac{l_{n-1} l_{n}}{\bar{l}_{n-1}}, \gamma_{i}(l)=\frac{l_{i} \bar{l}_{i+1}}{\bar{l}_{i} l_{i+1}} \\
e_{0}^{c}(l) & =\left(\frac{l_{1}}{\xi_{2}}, \frac{\xi_{2} l_{2}}{c}, \ldots, \xi_{2} \bar{l}_{2}, \frac{c \bar{l}_{1}}{\xi_{2}}\right), e_{n-1}^{c}(l)=\left(\ldots, c l_{n-1}, \frac{l_{n}}{c}, \ldots\right), \\
e_{n}^{c}(l) & \left.=\left(\ldots, c l_{n}, \frac{\bar{l}_{n-1}}{c}, \ldots\right), \quad \bar{l}_{i}, \ldots\right), \\
e_{i}^{c}(l) & =\left(\ldots, \frac{c l_{i}}{\xi_{i+1}}, \frac{\xi_{i+1} l_{i+1}}{c}, \ldots, \xi_{i+1} \bar{l}_{i+1}, \frac{\bar{\xi}_{i+1}}{\xi_{i}}, \ldots=\frac{\bar{l}_{i}+l_{i}}{\bar{l}_{i}+l_{i}}\right)(i=1, \ldots, n-2) .
\end{aligned}
$$

This is isomorphic to the geometric crystal $\mathcal{V}\left(D_{n}^{(1)}\right)_{L}$.

Proposition 6.1. We have the following isomorphism of $D_{n}^{(1)}$-geometric crystals:

$$
\mathcal{B}_{L}\left(D_{n}^{(1)}\right) \stackrel{\sim}{\longrightarrow} \mathcal{V}\left(D_{n}^{(1)}\right)_{L} .
$$

Proof. Define $\Xi: \mathcal{B}_{L}\left(D_{n}^{(1)}\right) \rightarrow \mathcal{V}\left(D_{n}^{(1)}\right)_{L}(l \mapsto x)$ to be

$$
\begin{gathered}
x_{i}:=\frac{1}{\bar{l}_{1} \bar{l}_{2} \cdots \bar{l}_{i}}, \bar{x}_{i}:=\frac{l_{1} \cdots l_{i}}{L}(i=1 \ldots, n-2), \\
x_{n-1}:=\frac{1}{\bar{l}_{1} \bar{l}_{2} \cdots \bar{l}_{n-1} l_{n}}, x_{n}:=\frac{1}{\bar{l}_{1} \bar{l}_{2} \cdots \bar{l}_{n-1}} .
\end{gathered}
$$

The inverse $\Xi^{-1}$ is given by

$$
\begin{gathered}
l_{1}=L \bar{x}_{1}, \bar{l}_{1}=\frac{1}{x_{1}}, l_{i}=\frac{\bar{x}_{i}}{\bar{x}_{i-1}}, \bar{l}_{i}=\frac{x_{i-1}}{x_{i}}(i=2, \ldots, n-2), \\
l_{n-1}=\frac{x_{n-1}}{\bar{x}_{n-2}}, l_{n}=\frac{x_{n}}{x_{n-1}}, \bar{l}_{n-1}=\frac{x_{n-2}}{x_{n}} .
\end{gathered}
$$

Then it is easy to see that it commutes with the action of $e_{i}$ and preserves $\gamma_{i}$ and $\varepsilon_{i}$. For example, for $x=\Xi(l)$, we have

$$
\xi_{2}=\frac{c \bar{l}_{2}+l_{2}}{\bar{l}_{2}+l_{2}}=\frac{c \frac{x_{1}}{x_{2}}+\frac{\bar{x}_{2}}{\bar{x}_{1}}}{\frac{x_{1}}{x_{2}}+\frac{\bar{x}_{2}}{\bar{x}_{1}}}=\frac{c x_{1} \bar{x}_{1}+x_{2} \bar{x}_{2}}{x_{1} \bar{x}_{1}+x_{2} \bar{x}_{2}}=: \Gamma_{1}
$$


Then we obtain

$$
\begin{aligned}
& \Xi\left(e_{0}^{c}(l)\right)=\Xi\left(\frac{l_{1}}{\xi_{2}}, \frac{\xi_{2} l_{2}}{c}, \ldots, \xi_{2} \bar{l}_{2}, \frac{c \bar{l}_{1}}{\xi_{2}}\right)=\left(\frac{c x_{1}}{\Gamma_{1}}, \frac{x_{2}}{c}, \ldots, \frac{\bar{x}_{2}}{c}, \Gamma_{1} \bar{x}_{1}\right)=e_{0}^{c} \Xi(l), \\
& \varepsilon_{0}(\Xi(l))=\varepsilon_{0}(x)=L \frac{x_{1} \bar{x}_{1}+x_{2} \bar{x}_{2}}{x_{1}}=L \frac{\frac{l_{1}}{L \bar{l}_{1}}+\frac{l_{1} l_{2}}{L \bar{l}_{1} \bar{l}_{2}}}{\frac{1}{\bar{l}_{1}}}=l_{1}\left(\frac{l_{2}}{\bar{l}_{2}}+1\right)=\varepsilon_{0}(l) .
\end{aligned}
$$

Other cases are shown similarly.

6.2. Folding. We introduce certain involutions on $\mathcal{B}_{L}\left(D_{n}^{(1)}\right)$ corresponding to the Dynkin diagram automorphisms for $D_{n}^{(1)}$. Let us consider the following Dynkin diagram automorphisms for $D_{N}^{(1)}$ :

$$
\begin{array}{cl}
N=n+1 & \sigma_{0}^{(n)}: \alpha_{0} \leftrightarrow \alpha_{1}, \quad \alpha_{i} \mapsto \alpha_{i} \quad(i \neq 0,1), \\
N=n+1 & \sigma_{1}^{(n)}: \alpha_{n} \leftrightarrow \alpha_{n+1}, \quad \alpha_{i} \mapsto \alpha_{i} \quad(i \neq n, n+1), \\
N=2 n & \sigma_{2}^{(n)}: \alpha_{i} \leftrightarrow \alpha_{2 n-i} \quad(i=0,1, \ldots, 2 n) .
\end{array}
$$

For each involution $\sigma_{j}^{(n)}$ on $D_{N}^{(1)}(j=0,1,2)$, we define the involution $\Sigma_{j}^{(n)}$ on $\mathcal{B}_{L}\left(D_{N}^{(1)}\right)(j=0,1,2)$ as a unique solution $l^{\prime}$ of the equations for a given $l$ :

$$
\gamma_{\sigma_{j}^{(n)}(i)}\left(l^{\prime}\right)=\gamma_{i}(l), \quad \varepsilon_{\sigma_{j}^{(n)}(i)}\left(l^{\prime}\right)=\varepsilon_{i}(l) \quad(i=0,1, \ldots, N) .
$$

Then we get $\left(l^{\prime}:=\Sigma_{j}^{(n)}(l)\right)$

$$
\begin{aligned}
\Sigma_{0}^{(n)}: & \mathcal{B}_{L}\left(D_{n+1}^{(1)}\right) \longrightarrow \mathcal{B}_{L}\left(D_{n+1}^{(1)}\right), \\
& l_{1}^{\prime}=\bar{l}_{1}, \quad \bar{l}_{1}^{\prime}=l_{1}, \quad l_{i}^{\prime}=l_{i}, \quad \bar{l}_{i}^{\prime}=\bar{l}_{i}(i \neq 1) . \\
\Sigma_{1}^{(n)}: & \mathcal{B}_{L}\left(D_{n+1}^{(1)}\right) \longrightarrow \mathcal{B}_{L}\left(D_{n+1}^{(1)}\right), \\
& l_{n}^{\prime}=l_{n} l_{n+1}, \quad l_{n+1}^{\prime}=\frac{1}{l_{n+1}}, \quad \bar{l}_{n}^{\prime}=l_{n+1} \bar{l}_{n}, \quad l_{i}^{\prime}=l_{i}, \quad \bar{l}_{i}^{\prime}=\bar{l}_{i} \quad(i \neq, n, n+1) . \\
\Sigma_{2}^{(n)}: & \mathcal{B}_{L}\left(D_{2 n}^{(1)}\right) \longrightarrow \mathcal{B}_{L}\left(D_{2 n}^{(1)}\right) \\
& l_{1}^{\prime}=\frac{l_{2 n-1} \bar{l}_{2 n-1}}{l_{2 n-1}+\bar{l}_{2 n-1}}, \quad \bar{l}_{1}^{\prime}=\frac{l_{2 n} l_{2 n-1} \bar{l}_{2 n-1}}{l_{2 n-1}+\bar{l}_{2 n-1}}, \quad l_{2 n}^{\prime}=\frac{\bar{l}_{1}}{l_{1}}, \\
& l_{2 n-1}^{\prime}=\frac{l_{1} \bar{l}_{2}}{l_{2}}\left(\frac{l_{2}}{\bar{l}_{2}}+1\right), \quad \bar{l}_{2 n-1}^{\prime}=l_{1}\left(\frac{l_{2}}{\bar{l}_{2}}+1\right), \\
& l_{2 n-i}^{\prime}=\frac{l_{i} \bar{l}_{i}}{l_{i+1}}\left(\frac{l_{i+1}+\bar{l}_{i+1}}{l_{i}+\bar{l}_{i}}\right), \quad \bar{l}_{2 n-i}^{\prime}=\frac{l_{i} \bar{l}_{i}}{\bar{l}_{i+1}}\left(\frac{l_{i+1}+\bar{l}_{i+1}}{l_{i}+\bar{l}_{i}}\right) \quad(2 \leq i \leq 2 n-2) .
\end{aligned}
$$

Lemma 6.2. Let $\left(X,\left\{e_{i}\right\}_{i=1,2,3},\left\{\gamma_{i}\right\}_{i=1,2,3},\left\{\varepsilon_{i}\right\}_{i=1,2,3}\right)$ be a $A_{3}$-geometric crystal $\left(a_{i j}=-1\right.$ if $\left.|i-j|=1\right)$. and set $E_{1}^{c}:=e_{1}^{c} \circ e_{3}^{c}\left(=e_{3}^{c} \circ e_{1}^{c}\right)$. Then we have

$$
E_{1}^{c} e_{2}^{c^{2} d} E_{1}^{c d} e_{2}^{d}=e_{2}^{d} E_{1}^{c d} e_{2}^{c^{2} d} E_{1}^{c} \quad\left(c, d \in \mathbb{C}^{\times}\right), \quad \gamma_{2}\left(E_{1}^{c}(x)\right)=c^{-2} \gamma_{2}(x) .
$$

Proof. The second equation is easily obtained:

$$
\gamma_{2}\left(E_{1}^{c}(x)\right)=\gamma_{2}\left(e_{1}^{c} \circ e_{3}^{c}(x)\right)=c^{-1} \gamma_{2}\left(e_{3}^{c}(x)\right)=c^{-2} \gamma_{2}(x) \quad(x \in X) .
$$

The first one is derived as follows:

$$
\begin{aligned}
& E_{1}^{c} e_{2}^{c^{2} d} E_{1}^{c d} e_{2}^{d}=\left(e_{1}^{c} e_{3}^{c}\right) e_{2}^{c^{2} d}\left(e_{3}^{c d} e_{1}^{c d}\right) e_{2}^{d}=e_{1}^{c} e_{2}^{c d} e_{3}^{c^{2} d} e_{2}^{c} e_{1}^{c d} e_{2}^{d}=e_{1}^{c} e_{2}^{c d} e_{3}^{c^{2}} e_{1}^{d} e_{2}^{c d} e_{1}^{c} \\
& =e_{1}^{c} e_{2}^{c d} e_{1}^{d} e_{3}^{c^{2} d} e_{2}^{c d} e_{1}^{c}=e_{2}^{d} e_{1}^{c d} e_{2}^{c} e_{3}^{c^{2} d} e_{2}^{c d} e_{1}^{c}=e_{2}^{d}\left(e_{1}^{c d} e_{3}^{c d}\right) e_{2}^{c^{2} d}\left(e_{3}^{c} e_{1}^{c}\right)=e_{2}^{d} E_{1}^{c d} e_{2}^{c^{2} d} E_{1}^{c}
\end{aligned}
$$


6.3. Fixed-point variety- $B_{n}^{(1)}$. As for the involution $\Sigma_{1}^{(n)}$ we consider the fixedpoint variety $X_{1}^{(n, L)} \subset \mathcal{B}_{L}\left(D_{n+1}^{(1)}\right)$ :

$$
X_{1}^{(n, L)}:=\left\{l=\left(l_{1}, \ldots, l_{n+1}, \bar{l}_{n}, \ldots, \bar{l}_{1}\right) \mid \Sigma_{1}^{(n)}(l)=l\left(\Leftrightarrow l_{n+1}=1\right)\right\} .
$$

Proposition 6.3. Let $\mathcal{B}_{L}\left(D_{n+1}^{(1)}\right)=\left(\mathcal{B}_{L},\left\{e_{i}\right\},\left\{\gamma_{i}\right\},\left\{\varepsilon_{i}\right\}\right)$ be the $D_{n+1}^{(1)}$-geometric crystal as above. Then we have a $B_{n}^{(1)}$-geometric crystal structure on $X_{1}^{(n, L)}$ as follows:

$\left(e_{i}^{\left(B_{n}^{(1)}\right)}\right)^{c}:=\left\{\begin{array}{ll}e_{i}^{c} & \text { for } i \neq n, \\ e_{n}^{c} \circ e_{n+1}^{c} & \text { for } i=n,\end{array} \quad \gamma_{i}^{\left(B_{n}^{(1)}\right):=\gamma_{i},} \quad \varepsilon_{i}^{\left(B_{n}^{(1)}\right)}:=\varepsilon_{i} \quad(i=0, \ldots, n)\right.$.

Proof. It suffices to check the conditions for a geometric crystal. But, most are trivial except the cases related to $i=n$. Namely, we have to see the Verma relation between $e_{n-1}$ and $e_{n}, \gamma_{i}^{\left(B_{n}^{(1)}\right)}\left(\left(e_{n}^{B_{n}^{(1)}}\right)^{c}(x)\right), \gamma_{n}^{\left(B_{n}^{(1)}\right)}\left(\left(e_{i}^{B_{n}^{(1)}}\right)^{c}(x)\right)$ and $\varepsilon_{n}^{\left(B_{n}^{(1)}\right)}\left(\left(e_{n}^{B_{n}^{(1)}}\right)^{c}(x)\right)$, which are immediate from Lemma 6.2

Moreover, the geometric crystal $X_{1}^{(n, L)}$ induces another $B_{n}^{(1)}$ - geometric crystal $\mathcal{B}_{L}\left(B_{n}^{(1)}\right)$ :

$$
\begin{aligned}
& \mathcal{B}_{L}\left(B_{n}^{(1)}\right):=\left\{m=\left(m_{1}, \ldots, m_{n}, \bar{m}_{n}, \ldots, \bar{m}_{1}\right)\left(\mathbb{C}^{\times}\right)^{2 n} \mid m_{1} \cdots m_{n} \bar{m}_{n} \cdots \bar{m}_{1}=L\right\}, \\
& \varepsilon_{0}(m)=m_{1}\left(\frac{m_{2}}{\bar{m}_{2}}+1\right), \varepsilon_{n}(m)=\bar{m}_{n}, \varepsilon_{i}(m)=\bar{m}_{i}\left(\frac{m_{i+1}}{\bar{m}_{i+1}}+1\right)(i=1, \ldots, n-1), \\
& \gamma_{0}(m)=\frac{\bar{m}_{1} \bar{m}_{2}}{m_{1} m_{2}}, \gamma_{n}(m)=\frac{m_{n}}{\bar{m}_{n}}, \gamma_{i}(m)=\frac{m_{i} \bar{m}_{i+1}}{\bar{m}_{i} m_{i+1}}(i=1, \ldots, n-1), \\
& e_{0}^{c}(m)=\left(\frac{m_{1}}{\xi_{2}}, \frac{\xi_{2} m_{2}}{c}, \ldots, \xi_{2} \bar{m}_{2}, \frac{c \bar{m}_{1}}{\xi_{2}}\right), e_{n}^{c}(m)=\left(\cdot ., c m_{n}, \frac{\bar{m}_{n}}{c}, \cdot .\right), \\
& e_{i}^{c}(m)=\left(\cdots, \frac{c m_{i}}{\xi_{i+1}}, \frac{\xi_{i+1} m_{i+1}}{c}, \ldots, \xi_{i+1} \bar{m}_{i+1}, \frac{\bar{m}_{i}}{\xi_{i+1}}, \ldots\right) \\
& (i=1, \ldots, n-1) \quad\left(\xi_{i}:=\frac{c \bar{m}_{i}+m_{i}}{\bar{m}_{i}+m_{i}}\right) .
\end{aligned}
$$

Let $\eta: \mathcal{B}_{L}\left(B_{n}^{(1)}\right) \longrightarrow X_{1}^{(n, L)}(m \mapsto l)$ be the morphism defined by

$$
l_{i}=m_{i}, \quad \bar{l}_{i}=\bar{m}_{i} \quad(i=1, \cdots, n),
$$

where $\left(l_{1}, \ldots, l_{n}, 1, \bar{l}_{n}, \ldots, \bar{l}_{1}\right) \in X_{1}^{(n, L)}$ and $m=\left(m_{1}, \ldots, m_{n}, \bar{m}_{n}, \bar{m}_{1}\right) \in \mathcal{B}_{L}\left(B_{n}^{(1)}\right)$. It is trivial that $\eta$ commutes with the actions $e_{i}$ and preserves $\gamma_{i}$ and $\varepsilon_{i}$. Then $\eta$ is an isomorphism of $B_{n}^{(1)}$-geometric crystals.

Proposition 6.4. We have the following isomorphisms of $B_{n}^{(1)}$-geometric crystals:

$$
\mathcal{V}\left(B_{n}^{(1)}\right)_{L} \stackrel{\sim}{\sim} \mathcal{B}_{L}\left(B_{n}^{(1)}\right) \stackrel{\sim}{\longrightarrow} X_{1}^{(n, L)} .
$$

Proof. The second isomorphism in (6.3) is given by $\eta$. Then we see the first one. Define $\Xi: \mathcal{B}_{L}\left(B_{n}^{(1)}\right) \rightarrow \mathcal{V}\left(B_{n}^{(1)}\right)_{L}(m \mapsto x)$ to be

$$
x_{i}:=\frac{1}{\bar{m}_{1} \bar{m}_{2} \cdots \bar{m}_{i}}(i=1, \ldots, n), \quad \bar{x}_{i}=\frac{m_{1} \cdots m_{i}}{L}(i=1, \ldots, n-1),
$$


and the inverse is

$$
\begin{gathered}
m_{1}=L \bar{x}_{1}, \quad m_{i}=\frac{\bar{x}_{i}}{\bar{x}_{i-1}}(2 \leq i \leq n-1), \\
m_{n}=\frac{x_{n}}{\bar{x}_{n-1}}, \quad \bar{m}_{1}=\frac{1}{x_{1}}, \quad \bar{m}_{i}=\frac{x_{i-1}}{x_{i}}(2 \leq i \leq n) .
\end{gathered}
$$

Then, by a direct calculation, we can check that $\Xi$ commutes with any $e_{i}^{c}$ and preserves $\gamma_{i}$ and $\varepsilon_{i}$. Thus, $\Xi$ is an isomorphism of $B_{n}^{(1)}$-geometric crystals.

6.4. Fixed-point variety- $D_{n+1}^{(2)}$. As for the involution

$$
\Sigma_{3}^{(n)}:=\Sigma_{0}^{(n+1)} \circ \Sigma_{1}^{(n+1)}\left(=\Sigma_{1}^{(n+1)} \circ \Sigma_{0}^{(n+1)}\right)
$$

on $\mathcal{B}_{L}\left(D_{n+2}^{(1)}\right)$, we consider the fixed-point variety $X_{3}^{(n, L)} \subset \mathcal{B}_{L}\left(D_{n+2}^{(1)}\right)$ :

$$
X_{3}^{(n, L)}:=\left\{l=\left(l_{1}, \ldots, l_{n+2}, \bar{l}_{n+1}, \ldots, \bar{l}_{1}\right) \mid \Sigma_{3}^{(n, L)}(l)=l\left(\Leftrightarrow l_{n+2}=1, l_{1}=\bar{l}_{1}\right)\right\} .
$$

Proposition 6.5. Let $\mathcal{B}_{L}\left(D_{n+2}^{(1)}\right)=\left(\mathcal{B}_{L},\left\{e_{i}\right\},\left\{\gamma_{i}\right\},\left\{\varepsilon_{i}\right\}\right)$ be the $D_{n+2}^{(1)}$-geometric crystal as above. Then we have a $D_{n+1}^{(2)}$-geometric crystal structure on $X_{3}^{(n, L)}$ as follows:

$$
\left(e_{i}^{\left(D_{n+1}^{(2)}\right)}\right)^{c}:= \begin{cases}e_{i+1}^{c} & \text { for } i \neq 0, n, \\ e_{0}^{c} \circ e_{1}^{c} & \text { for } i=0, \\ & \gamma_{i}^{\left(D_{n+1}^{(2)}\right)}:=\gamma_{i+1}, \varepsilon_{i}^{\left(D_{n+1}^{(2)}\right)}:=\varepsilon_{i+1} \quad(i=0, \ldots, n) . \\ e_{n+1}^{c} \circ e_{n+2}^{c} & \text { for } i=n,\end{cases}
$$

Proof. We can prove it by the similar argument for $X_{1}^{(n, L)}$ using Lemma 6.2.

The geometric crystal $X_{3}^{(n, L)}$ induces another $D_{n+1}^{(2)}$-geometric crystal $\mathcal{B}_{L}\left(D_{n+1}^{(2)}\right)$ :

$$
\begin{aligned}
& \mathcal{B}_{L}\left(D_{n+1}^{(2)}\right):=\left\{m=\left(m_{0}, m_{1}, \ldots, m_{n}, \bar{m}_{n}, \ldots, \bar{m}_{1}\right)\left(\mathbb{C}^{\times}\right)^{2 n} \mid m_{0}^{2} m_{1} \ldots\right. \\
& \varepsilon_{0}(m)=m_{0}\left(\frac{m_{1}}{\bar{m}_{1}}+1\right), \varepsilon_{n}(m)=\bar{m}_{n}, \varepsilon_{i}(m)=\bar{m}_{i}\left(\frac{m_{i+1}}{\bar{m}_{i+1}}+1\right) \quad(i=1, \ldots, n-1), \\
& \gamma_{0}(m)=\frac{\bar{m}_{1}}{m_{1}}, \gamma_{n-1}(m)=\frac{m_{n-1}}{m_{n} \bar{m}_{n-1}}, \gamma_{n}(m)=\frac{m_{n}}{\bar{m}_{n}}, \\
& \gamma_{i}(m)=\frac{m_{i} \bar{m}_{i+1}}{\bar{m}_{i} m_{i+1}}(i=1, \ldots, n-2), \\
& e_{0}^{c}(m)=\left(\frac{c m_{0}}{\xi_{1}}, \frac{\xi_{1} m_{1}}{c^{2}}, \ldots, \xi_{1} \bar{m}_{1}\right)\left(\xi_{1}(m):=\frac{c^{2} \bar{m}_{1}+m_{1}}{\bar{m}_{1}+m_{1}}\right), \\
& e_{n}^{c}(m)=\left(\ldots, c m_{n}, \frac{\bar{m}_{n}}{c}, \ldots\right), \\
& e_{i}^{c}(m)=\left(\ldots, \frac{c m_{i}}{\xi_{i+1}}, \frac{\xi_{i+1} m_{i+1}}{c}, \ldots, \xi_{i+1} \bar{m}_{i+1}, \frac{\bar{m}_{i}}{\xi_{i+1}}, \ldots\right) \quad(i=1, \ldots, n-1) \\
& \quad\left(\xi_{i}:=\frac{c \bar{m}_{i}+m_{i}}{\bar{m}_{i}+m_{i}}\right) .
\end{aligned}
$$

Let $\eta: \mathcal{B}_{L}\left(D_{n+1}^{(2)}\right) \longrightarrow X_{3}^{(n, L)}(m \mapsto l)$ be the morphism defined by

$$
l_{i+1}=m_{i} \quad(i=0,1, \cdots, n), \quad \bar{l}_{i+1}=\bar{m}_{i} \quad(i=1, \cdots, n),
$$


where $\left(l_{1}, \ldots, l_{n+1}, 1, \bar{l}_{n+1}, \ldots, \bar{l}_{2}, l_{1}\right) \in X_{3}^{(n, L)}$ and $m=\left(m_{0}, m_{1}, \ldots, m_{n}, \bar{m}_{n}, \bar{m}_{1}\right)$ $\in \mathcal{B}_{L}\left(D_{n+1}^{(2)}\right)$. Then, $\eta$ is an isomorphism of $D_{n+1}^{(2)}$-geometric crystal.

Proposition 6.6. We have the following isomorphisms of $D_{n+1}^{(2)}$-geometric crystals:

$$
\mathcal{V}\left(D_{n+1}^{(2)}\right)_{L} \stackrel{\sim}{\longleftarrow} \mathcal{B}_{L^{2}}\left(D_{n+1}^{(2)}\right) \stackrel{\sim}{\longrightarrow} X_{3}^{\left(n, L^{2}\right)}
$$

Proof. The second isomorphism in (6.4) is given by $\eta$. Then we see the first one. Define $\Xi: \mathcal{B}_{L^{2}}\left(D_{n+1}^{(2)}\right) \rightarrow \mathcal{V}\left(D_{n+1}^{(2)}\right)_{L}(m \mapsto x)$ to be

$x_{0}:=\frac{1}{m_{0}}, \quad x_{i}:=\frac{1}{m_{0}^{2} \bar{m}_{1} \bar{m}_{2} \cdots \bar{m}_{i}}(i=1, \ldots, n), \quad \bar{x}_{i}=\frac{m_{1} \cdots m_{i}}{L^{2}}(i=1, \ldots, n-1)$.

and the inverse is

$$
\begin{gathered}
m_{0}=\frac{1}{x_{0}}, m_{1}=L^{2} \bar{x}_{1}, m_{i}=\frac{\bar{x}_{i}}{\bar{x}_{i-1}}(2 \leq i \leq n-1), \\
m_{n}=\frac{x_{n}}{\bar{x}_{n-1}}, \bar{m}_{1}=\frac{x_{0}^{2}}{x_{1}}, \bar{m}_{i}=\frac{x_{i-1}}{x_{i}}(2 \leq i \leq n) .
\end{gathered}
$$

Then, calculating directly, we can check that $\Xi$ is an isomorphism of geometric crystals.

6.5. Fixed-point variety- $A_{2 n-1}^{(2)}$. As for the involution $\Sigma_{2}^{(n)}$ we consider the fixedpoint variety $X_{2}^{(n, L)} \subset \mathcal{B}_{L}\left(D_{2 n}^{(1)}\right)$ :

$$
X_{2}^{(n, L)}:=\left\{l=\left(l_{1}, \ldots, l_{2 n}, \bar{l}_{2 n-1}, \cdots, \bar{l}_{1}\right) \mid \Sigma_{2}^{(n)}(l)=l\right\}
$$

where the condition $\Sigma_{2}^{(n)}(l)=l$ is equivalent to

$$
\begin{aligned}
& l_{1}=\frac{l_{2 n-1} \bar{l}_{2 n-1}}{l_{2 n-1}+\bar{l}_{2 n-1}}, \quad \bar{l}_{1}=\frac{l_{2 n} l_{2 n-1} \bar{l}_{2 n-1}}{l_{2 n-1}+\bar{l}_{2 n-1}} \\
& l_{2 n-1}=\frac{l_{1} \bar{l}_{2}}{l_{2}}\left(\frac{l_{2}}{\bar{l}_{2}}+1\right), \quad \bar{l}_{2 n-1}=l_{1}\left(\frac{l_{2}}{\bar{l}_{2}}+1\right), \\
& l_{2 n-i}=\frac{l_{i} \bar{l}_{i}}{l_{i+1}} \frac{l_{i+1}+\bar{l}_{i+1}}{l_{i}+\bar{l}_{i}}, \quad \bar{l}_{2 n-i}=\frac{l_{i} \bar{l}_{i}}{\bar{l}_{i+1}} \frac{l_{i+1}+\bar{l}_{i+1}}{l_{i}+\bar{l}_{i}} \quad(i=2, \ldots, 2 n-2), \quad l_{2 n}=\frac{\bar{l}_{1}}{l_{1}} .
\end{aligned}
$$

Note that all of the equations in (6.5) are not necessarily independent. Indeed, we only need $2 n$-equations: $l_{1}=\cdots, l_{n}=\cdots$ and $\bar{l}_{1}=\cdots, \bar{l}_{n}=\cdots$ in (6.5).

Proposition 6.7. Let $\mathcal{B}_{L}\left(D_{2 n}^{(1)}\right)=\left(\mathcal{B}_{L},\left\{e_{i}\right\},\left\{\gamma_{i}\right\},\left\{\varepsilon_{i}\right\}\right)$ be the $D_{2 n}^{(1)}$-geometric crystal as above. Then we have a $A_{2 n-1}^{(2)}$-geometric crystal structure on $X_{2}^{(n, L)}$ as follows:

$$
\left(e_{i}^{\left(A_{2 n-1}^{(2)}\right)}\right)^{c}:=\left\{\begin{array}{lll}
e_{i}^{c} \circ e_{2 n-i}^{c} & \text { for } 0 \leq i \leq n-1, & \gamma_{i}^{\left(A_{2 n-1}^{(2)}\right)}:=\gamma_{i}\left(=\gamma_{2 n-i}\right), \\
e_{n}^{c} & \text { for } i=n, & \varepsilon_{i}^{\left(A_{2 n-1}^{(2)}\right)}:=\varepsilon_{i}\left(=\varepsilon_{2 n-i}\right) \quad(i=0, \ldots, n) .
\end{array}\right.
$$

The proof is similar to the previous cases. 
The geometric crystal $X_{2}^{(n, L)}$ induces another $A_{2 n-1}^{(2)}$-geometric crystal $\mathcal{B}_{L}\left(A_{2 n-1}^{(2)}\right):$

$$
\begin{aligned}
\mathcal{B}_{L}\left(A_{2 n-1}^{(2)}\right):=\{m & =\left(m_{1}, \ldots, m_{n}, \bar{m}_{n}, \ldots, \bar{m}_{1}\right) \\
& \left.\in\left(\mathbb{C}^{\times}\right)^{2 n} \mid m_{n} \bar{m}_{n}\left(m_{1} \cdots m_{n-1} \bar{m}_{n-1} \cdots \bar{m}_{1}\right)^{2}=L^{2}\right\} .
\end{aligned}
$$

For $m \in \mathcal{B}_{L}\left(A_{2 n-1}^{(2)}\right)$ set

$$
\begin{aligned}
& \mu(m):=\frac{L}{m_{1} \cdots m_{n-1} \bar{m}_{n} \cdots \bar{m}_{1}}, \quad \bar{\mu}(m):=\frac{L}{m_{1} \cdots m_{n} \bar{m}_{n-1} \cdots \bar{m}_{1}}\left(=\frac{1}{\mu(m)}\right), \\
& \varepsilon_{0}(m)=m_{1}\left(\frac{m_{2}}{\bar{m}_{2}}+1\right), \varepsilon_{n-1}(m)=\bar{m}_{n-1}(\mu(m)+1), \\
& \varepsilon_{n}(m)=\bar{m}_{n}, \varepsilon_{i}(m)=\bar{m}_{i}\left(\frac{m_{i+1}}{\bar{m}_{i+1}}+1\right), \\
& \gamma_{0}(m)=\frac{\bar{m}_{1} \bar{m}_{2}}{m_{1} m_{2}}, \gamma_{n-1}(m)=\frac{m_{n-1}}{\bar{m}_{n-1}} \bar{\mu}(m), \gamma_{n}(m)=\frac{m_{n}}{\bar{m}_{n}}, \\
& \gamma_{i}(m)=\frac{m_{i} \bar{m}_{i+1}}{\bar{m}_{i} m_{i+1}}(1 \leq i \leq n-2), \\
& e_{0}^{c}(m)=\left(\frac{m_{1}}{\xi_{1}}, \frac{\xi_{1} m_{2}}{c}, \ldots, \xi_{1} \bar{m}_{2}, \frac{c \bar{m}_{1}}{\xi_{1}}\right), e_{n}^{c}(m)=\left(\ldots, c m_{n}, \frac{\bar{m}_{n}}{c}, \ldots\right), \\
& e_{n-1}^{c}(m)=\left(\ldots, m_{n-1} \frac{c}{\xi_{n-1}}, m_{n} \frac{\xi_{n-1}^{2}}{c^{2}}, \bar{m}_{n} \xi_{n-1}^{2}, \frac{\bar{m}_{n-1}}{\xi_{n-1}}, \ldots\right), \\
& e_{i}^{c}(m)=\left(\ldots, \frac{c m_{i}}{\xi_{i}}, \frac{\xi_{i} m_{i+1}}{c}, \ldots, \xi_{i} \bar{m}_{i+1}, \frac{\bar{m}_{i}}{\xi_{i}}, \ldots\right) \quad(i=1, \ldots, n-1),
\end{aligned}
$$

where

$$
\xi_{i}:= \begin{cases}\frac{c \bar{m}_{i+1}+m_{i+1}}{\bar{m}_{i+1}+m_{i+1}} & \text { for } i \neq n-1, \\ \frac{c+\mu(m)}{1+\mu(m)} & \text { for } i=n-1 .\end{cases}
$$

Let $\eta: \mathcal{B}_{L}\left(A_{2 n-1}^{(2)}\right) \longrightarrow X_{2}^{\left(n, L^{2}\right)}(m \mapsto l)$ be the morphism defined by

$$
l_{i}=m_{i}, \quad \bar{l}_{i}=\bar{m}_{i} \quad(i=1, \ldots, n-1), \quad l_{n}=\frac{m_{n}}{1+\mu(m)}, \quad \bar{l}_{n}=\frac{\bar{m}_{n}}{1+\bar{\mu}(m)},
$$

where $l_{i}, \bar{l}_{i}(i=n+1, \ldots, 2 n)$ are uniquely determined by (6.5) and then, $\eta$ is an isomorphism of $A_{2 n-1}^{(2)}$-geometric crystal. 
Proposition 6.8. We have the following isomorphisms of $A_{2 n-1}^{(2)}$-geometric crystals:

$$
\mathcal{V}\left(A_{2 n-1}^{(2)}\right)_{L} \stackrel{\sim}{\sim} \mathcal{B}_{L}\left(A_{2 n-1}^{(2)}\right) \stackrel{\sim}{\longrightarrow} X_{2}^{\left(n, L^{2}\right)} .
$$

Proof. The second isomorphism in (6.6) is given by $\eta$. Then let us check the first one. Define $\Xi: \mathcal{B}_{L}\left(A_{2 n-1}^{(2)}\right) \rightarrow \mathcal{V}\left(A_{2 n-1}^{(2)}\right)_{L}(m \mapsto x)$ to be

$x_{i}:=\frac{1}{\bar{m}_{1} \bar{m}_{2} \cdots \bar{m}_{i}}, \quad \bar{x}_{i}=\frac{m_{1} \cdots m_{i}}{L^{2}}(i=1, \ldots, n-1), \quad x_{n}=\frac{1}{\bar{m}_{n}\left(\bar{m}_{1} \cdots \bar{m}_{n-1}\right)^{2}}$,

and then the inverse is

$$
\begin{gathered}
m_{1}=L^{2} \bar{x}_{1}, \quad \bar{m}_{1}=\frac{1}{x_{1}}, \quad m_{i}=\frac{\bar{x}_{i}}{\bar{x}_{i-1}}, \quad \bar{m}_{i}=\frac{x_{i-1}}{x_{i}}(2 \leq i \leq n-1), \\
m_{n}=\frac{x_{n}}{L^{2} \bar{x}_{n-1}^{2}}, \quad \bar{m}_{n}=\frac{x_{n-1}^{2}}{x_{n}} .
\end{gathered}
$$

Then, by direct calculations, we can check that $\Xi$ is an isomorphism of geometric crystals.

6.6. Fixed-point variety- $A_{2 n}^{(2)}$. As for the involution $\Sigma_{4}^{(n)}:=\Sigma_{2}^{(n+1)} \Sigma_{1}^{(2 n+1)} \Sigma_{0}^{(2 n+1)}$ we consider a fixed-point variety $X_{4}^{(n, L)} \subset \mathcal{B}_{L}\left(D_{2 n+2}^{(1)}\right)$ :

$$
X_{4}^{(n, L)}:=\left\{l=\left(l_{1}, \ldots, l_{2 n+2}, \bar{l}_{2 n+1}, \cdots, \bar{l}_{1}\right) \mid \Sigma_{4}^{(n)}(l)=l\right\},
$$

where the condition $\Sigma_{4}^{(n)}(l)=l$ is equivalent to

$$
\begin{aligned}
& l_{1}=\bar{l}_{1}=\frac{l_{2 n+1} \bar{l}_{2 n+1}}{l_{2 n+1}+\bar{l}_{2 n+1}}, \quad l_{2 n+1}=\frac{l_{1} \bar{l}_{2}}{l_{2}}\left(\frac{l_{2}}{\bar{l}_{2}}+1\right), \quad \bar{l}_{2 n+1}=l_{1}\left(\frac{l_{2}}{\bar{l}_{2}}+1\right), \quad l_{2 n+2}=1, \\
& l_{2 n+2-i}=\frac{l_{i} \bar{l}_{i}}{l_{i+1}} \frac{l_{i+1}+\bar{l}_{i+1}}{l_{i}+\bar{l}_{i}}, \quad \bar{l}_{2 n+2-i}=\frac{l_{i} \bar{l}_{i}}{\bar{l}_{i+1}} \frac{l_{i+1}+\bar{l}_{i+1}}{l_{i}+\bar{l}_{i}} \quad(i=2, \ldots, 2 n) .
\end{aligned}
$$

Note that all the equations in (6.7) are not necessarily independent. Indeed, we only need $2 n$-equations: $l_{1}=\cdots, l_{n}=\cdots$ and $\bar{l}_{1}=\cdots, \bar{l}_{n}=\cdots$ in (6.7).

Proposition 6.9. Let $\mathcal{B}_{L}\left(D_{2 n+2}^{(1)}\right)=\left(\mathcal{B}_{L},\left\{e_{i}\right\},\left\{\gamma_{i}\right\},\left\{\varepsilon_{i}\right\}\right)$ be the $D_{2 n+2}^{(1)}$-geometric crystal as above. Then we have a $A_{2 n}^{(2)}$-geometric crystal structure on $X_{4}^{(n, L)}$ as follows:

$$
\begin{aligned}
\left(e_{i}^{\left(A_{2 n}^{(2)}\right)}\right)^{c} & := \begin{cases}e_{0}^{c} \circ e_{1}^{c} \circ e_{2 n+1}^{c} \circ e_{2 n+2}^{c} & \text { for } i=0, \\
e_{i+1}^{c} \circ e_{2 n-i+1}^{c} & \text { for } 1 \leq i \leq n-1, \\
e_{n+1}^{c} & \text { for } i=n,\end{cases} \\
\gamma_{i}^{\left(A_{2 n}^{(2)}\right)}: & = \begin{cases}\gamma_{0}\left(=\gamma_{1}=\gamma_{2 n+1}=\gamma_{2 n+2}\right) & \text { for } i=0, \\
\gamma_{i+1}\left(=\gamma_{2 n-i+1}\right) & \text { for } i \neq 0,\end{cases} \\
\varepsilon_{i}^{\left(A_{2 n}^{(2)}\right)}: & = \begin{cases}\varepsilon_{0}\left(=\varepsilon_{1}=\varepsilon_{2 n+1}=\varepsilon_{2 n+2}\right) & \text { for } i=0, \\
\varepsilon_{i+1}\left(=\varepsilon_{2 n-i+1}\right) & \text { for } i \neq 0 .\end{cases}
\end{aligned}
$$

We can prove it by arguments similar to those of the previous cases.

The geometric crystal $X_{4}^{(n, L)}$ induces another $A_{2 n}^{(2)}$-geometric crystal $\mathcal{B}_{L}\left(A_{2 n}^{(2)}\right)$ :

$$
\begin{aligned}
\mathcal{B}_{L}\left(A_{2 n}^{(2)}\right):=\{m= & \left(m_{0}, m_{1}, \ldots, m_{n}, \bar{m}_{n}, \ldots, \bar{m}_{1}\right) \\
& \left.\in\left(\mathbb{C}^{\times}\right)^{2 n} \mid m_{n} \bar{m}_{n}\left(m_{0}^{2} m_{1} \cdots m_{n-1} \bar{m}_{n-1} \cdots \bar{m}_{1}\right)^{2}=L^{2}\right\} .
\end{aligned}
$$


For $m \in \mathcal{B}_{L}\left(A_{2 n}^{(2)}\right)$ set

$$
\begin{aligned}
& \mu(m):=\frac{L}{m_{0}^{2} m_{1} \cdots m_{n-1} \bar{m}_{n} \cdots \bar{m}_{1}}, \quad \bar{\mu}(m):=\frac{L}{m_{0}^{2} m_{1} \cdots m_{n} \bar{m}_{n-1} \cdots \bar{m}_{1}}\left(=\frac{1}{\mu(m)}\right) . \\
& \varepsilon_{0}(m)=m_{0}\left(\frac{m_{1}}{\bar{m}_{1}}+1\right), \varepsilon_{n-1}(m)=\bar{m}_{n-1}(\mu(m)+1), \\
& \varepsilon_{n}(m)=\bar{m}_{n}, \varepsilon_{i}(m)=\bar{m}_{i}\left(\frac{m_{i+1}}{\bar{m}_{i+1}}+1\right), \\
& \gamma_{0}(m)=\frac{\bar{m}_{1}}{m_{1}}, \gamma_{n-1}(m)=\frac{m_{n-1}}{\bar{m}_{n-1}} \bar{\mu}(m), \gamma_{n}(m)=\frac{m_{n}}{\bar{m}_{n}}, \\
& \gamma_{i}(m)=\frac{m_{i} \bar{m}_{i+1}}{\bar{m}_{i} m_{i+1}}(1 \leq i \leq n-2), \\
& e_{0}^{c}(m)=e_{0}^{c}(m)=\left(\frac{c m_{0}}{\xi_{0}}, \frac{\xi_{0} m_{1}}{c^{2}}, \ldots, \xi_{0} \bar{m}_{1}\right), e_{n}^{c}(m)=\left(\ldots, c m_{n}, \frac{\bar{m}_{n}}{c}, \ldots\right), \\
& e_{n-1}^{c}(m)=\left(\ldots, m_{n-1} \frac{c}{\xi_{n-1}}, m_{n} \frac{\xi_{n-1}^{2}}{c^{2}}, \bar{m}_{n} \xi_{n-1}^{2}, \frac{\bar{m}_{n-1}}{\xi_{n-1}}, \ldots\right), \\
& e_{i}^{c}(m)=\left(\ldots, \frac{c m_{i}}{\xi_{i}}, \frac{\xi_{i} m_{i+1}}{c}, \ldots, \xi_{i} \bar{m}_{i+1}, \frac{\bar{m}_{i}}{\xi_{i}}, \ldots\right) \quad(1 \leq i \leq n-2),
\end{aligned}
$$

where

$$
\xi_{i}:= \begin{cases}\frac{c^{2} \bar{m}_{1}+m_{1}}{\bar{m}_{1}+m_{1}} & \text { for } i=0, \\ \frac{c m_{i+1}+m_{i+1}}{\bar{m}_{i+1}+m_{i+1}} & \text { for } i \neq 0, n-1 \\ \frac{c+\mu(m)}{1+\mu(m)} & \text { for } i=n-1 .\end{cases}
$$

Let $\eta: \mathcal{B}_{L}\left(A_{2 n}^{(2)}\right) \longrightarrow X_{4}^{\left(n, L^{2}\right)}(m \mapsto l)$ be the morphism defined by

$$
\begin{gathered}
l_{1}=\bar{l}_{1}=m_{0}, \quad l_{i}=m_{i-1}, \quad \bar{l}_{i}=\bar{m}_{i-1}(i=2, \ldots, n), \\
l_{n+1}=\frac{m_{n}}{1+\mu(m)}, \quad \bar{l}_{n+1}=\frac{\bar{m}_{n}}{1+\bar{\mu}(m)},
\end{gathered}
$$

where $l_{i}, \bar{l}_{i}(i=n+2, \ldots, 2 n+2)$ are uniquely determined by (6.7) and then, $\eta$ is an isomorphism of $A_{2 n}^{(2)}$-geometric crystal.

Proposition 6.10. We have the following isomorphisms of $A_{2 n}^{(2)}$-geometric crystals:

$$
\mathcal{V}\left(A_{2 n}^{(2)}\right)_{L} \stackrel{\sim}{\sim} \mathcal{B}_{L}\left(A_{2 n}^{(2)}\right) \stackrel{\sim}{\longrightarrow} X_{4}^{\left(n, L^{2}\right)} .
$$

Proof. The second isomorphism in (6.8) is given by $\eta$. Then we check the first one. Define $\Xi: \mathcal{B}_{L}\left(A_{2 n}^{(2)}\right) \rightarrow \mathcal{V}\left(A_{2 n-1}^{(2)}\right)_{L}(m \mapsto x)$ to be

$$
\begin{gathered}
x_{0}=\frac{1}{m_{0}}, \quad x_{i}:=\frac{1}{m_{0}^{2} \bar{m}_{1} \bar{m}_{2} \cdots \bar{m}_{i}}, \quad \bar{x}_{i}=\frac{m_{1} \cdots m_{i}}{L^{2}}(i=1, \ldots, n-1), \\
x_{n}=\frac{1}{\bar{m}_{n}\left(m_{0}^{2} \bar{m}_{1} \cdots \bar{m}_{n-1}\right)^{2}},
\end{gathered}
$$


and the inverse $\Xi^{-1}$ is

$$
\begin{aligned}
m_{0}=\frac{1}{x_{0}}, m_{1}=L^{2} \bar{x}_{1}, \bar{m}_{1} & =\frac{x_{0}^{2}}{x_{1}}, m_{i}=\frac{\bar{x}_{i}}{\bar{x}_{i-1}}, \bar{m}_{i}=\frac{x_{i-1}}{x_{i}}(2 \leq i \leq n-1), \\
m_{n} & =\frac{x_{n}}{\bar{x}_{n-1}^{2}}, \bar{m}_{n}=\frac{x_{n-1}^{2}}{x_{n}} .
\end{aligned}
$$

Then, by direct calculations, we can check that $\Xi$ is an isomorphism of geometric crystals.

\section{Product Structure on afFine geometric Crystals}

In general, if $\mathbb{X}_{1}$ and $\mathbb{X}_{2}$ are geometric crystals induced from unipotent crystals, the product $\mathbb{X}_{1} \times \mathbb{X}_{2}$ possesses a geometric crystal structure (BK], $\mathbb{\mathrm { N } 1}$ ). More precisely, let $\mathbb{X}_{1}=\left(X,\left\{e_{i}\right\},\left\{\gamma_{i}\right\},\left\{\varepsilon_{i}\right\}\right)$ and $\mathbb{X}_{2}=\left(Y,\left\{e_{i}\right\},\left\{\gamma_{i}\right\},\left\{\varepsilon_{i}\right\}\right)$ be geometric crystals. For $x \in X$ and $y \in Y$ set

$$
\begin{aligned}
& \gamma_{i}(x, y):=\gamma_{i}(x) \gamma_{i}(y), \\
& \varepsilon_{i}(x, y):=\varepsilon_{i}(x)+\frac{\varepsilon_{i}(x) \varepsilon_{i}(y)}{\varphi_{i}(x)} \quad\left(\varphi_{i}(x)=\gamma(x) \varepsilon_{i}(x)\right), \\
& e_{i}^{c}(x, y):=\left(e_{i}^{c_{1}}(x), e_{i}^{c_{2}}(y)\right) \quad \text { where } c_{1}:=\frac{c \varphi_{i}(x)+\varepsilon_{i}(y)}{\varphi_{i}(x)+\varepsilon_{i}(y)}, \quad c_{2}:=\frac{c}{c_{1}} .
\end{aligned}
$$

Theorem 7.1 (BK,, $\mathrm{N} 1)$. Suppose that the geometric crystals $\mathbb{X}_{1}$ and $\mathbb{X}_{2}$ are induced from unipotent crystals. Then, (7.1) -(7.3) endow the product $X \times Y$ with a structure of a geometric crystal. Moreover, if $\mathbb{X}_{1}$ and $\mathbb{X}_{2}$ are positive, then $\mathbb{X}_{1} \times \mathbb{X}_{2}$ is positive and we have the isomorphism of crystals:

$$
\mathcal{U} D\left(\mathbb{X}_{1} \times \mathbb{X}_{2}\right) \cong \mathcal{U} D\left(\mathbb{X}_{1}\right) \otimes \mathcal{U} D\left(\mathbb{X}_{2}\right) .
$$

As for the affine geometric crystal $\mathcal{V}(\mathfrak{g})_{l}$ in Section 5 , its data $e_{i}, \gamma_{i}, \varepsilon_{i}(i \in I \backslash\{0\})$ are obtained from the ones of the geometric crystal $B_{\mathbf{i}}^{-} \cdot l^{H}$ as in 2.3 which is induced from the unipotent crystal on some $X_{w} \times l^{H}$ where $\mathbf{i}$ is a reduced word for $w$ and $X_{w}$ is the Schubert cell associated with $w \in W$. Thus, by Theorem 7.1 we have a $\mathfrak{g}_{0}$-geometric crystal structure on $\mathcal{V}(\mathfrak{g})_{L_{1}} \times \cdots \times \mathcal{V}(\mathfrak{g})_{L_{k}}$.

Theorem 7.2. For any $k \in \mathbb{Z}_{\geq 0}$ and $L_{1}, \cdots, L_{k} \in \mathbb{C}^{\times}$, the product $\mathcal{V}(\mathfrak{g})_{L_{1}} \times \cdots \times$ $\mathcal{V}(\mathfrak{g})_{L_{k}}$ possesses an affine geometric crystal structure.

Proof. By the argument above and Theorem 7.1 it is enough to check the conditions in Definition 2.1 related to $i=0$. First, let us check $\gamma_{j}\left(e_{i}^{c}\left(x_{1}, \cdots, x_{k}\right)\right)=$ $c^{a_{i j}} \gamma_{j}\left(x_{1}, \cdots, x_{k}\right) \quad$ for $(i, j)=(i, 0),(0, i)$. For $x_{j} \in \mathcal{V}(\mathfrak{g})_{L_{j}}$ and $i \in I$, we have

$$
\begin{aligned}
\gamma_{0}\left(e_{i}^{c}\left(x_{1}, \cdots, x_{k}\right)\right) & =\gamma_{0}\left(e_{i}^{c_{1}}\left(x_{1}\right)\right) \cdots \gamma_{0}\left(e_{i}^{c_{k}}\left(x_{k}\right)\right) \\
& =c_{1}^{a_{i 0}} \gamma_{0}\left(x_{1}\right) \cdots c_{k}^{a_{i 0}} \gamma_{0}\left(x_{k}\right)=c^{a_{i 0}} \gamma_{0}\left(x_{1}, \cdots, x_{k}\right), \\
\gamma_{i}\left(e_{0}^{c}\left(x_{1}, \cdots, x_{k}\right)\right) & =\gamma_{i}\left(e_{0}^{c_{1}}\left(x_{1}\right)\right) \cdots \gamma_{i}\left(e_{0}^{c_{k}}\left(x_{k}\right)\right) \\
& =c_{1}^{a_{0 i}} \gamma_{i}\left(x_{1}\right) \cdots c_{k}^{a_{0 i}} \gamma_{i}\left(x_{k}\right)=c^{a_{0 i}} \gamma_{i}\left(x_{1}, \cdots, x_{k}\right),
\end{aligned}
$$

where $c_{1}, \cdots, c_{k}$ are obtained by using (7.3) repeatedly. 
Next, let us check the relation $\varepsilon_{0}\left(e_{0}^{c}\left(x_{1}, \cdots, x_{k}\right)\right)=c^{-1} \varepsilon_{0}\left(x_{1}, \cdots, x_{k}\right)$ by the induction on $k$. Assume $\varepsilon_{0}\left(e_{0}^{c}\left(x^{\prime}\right)\right)=c^{-1} \varepsilon_{0}\left(\left(x^{\prime}\right)\right)$ and $\varphi_{0}\left(e_{0}^{c}\left(x^{\prime}\right)\right)=c \varphi_{0}\left(\left(x^{\prime}\right)\right)$ where $x^{\prime}=\left(x_{1}, \cdots, x_{k-1}\right)$.

$$
\begin{aligned}
\varepsilon_{0}\left(e_{0}^{c}\left(x_{1}, \cdots, x_{k}\right)\right) & =\varepsilon_{0}\left(e_{0}^{c_{1}}\left(x^{\prime}\right), e_{0}^{c_{2}}\left(x_{k}\right)\right)=\varepsilon_{0}\left(e_{0}^{c_{1}}\left(x^{\prime}\right)\right)+\frac{\varepsilon_{0}\left(e_{0}^{c_{1}}\left(x^{\prime}\right)\right) \varepsilon_{0}\left(e_{0}^{c_{2}}\left(x_{k}\right)\right)}{\varphi_{0}\left(e_{0}^{c_{1}}\left(x^{\prime}\right)\right)} \\
& =c_{1}^{-1} \varepsilon_{0}\left(x^{\prime}\right)+\frac{\varepsilon_{0}\left(x^{\prime}\right) \varepsilon_{0}\left(x_{k}\right)}{c c_{1} \varphi_{0}\left(x^{\prime}\right)}=c_{1}^{-1}\left(\varepsilon_{0}\left(x^{\prime}\right)+\frac{\varepsilon_{0}\left(x^{\prime}\right) \varepsilon_{0}\left(x_{k}\right)}{c \varphi_{0}\left(x^{\prime}\right)}\right) \\
& =\frac{\varphi_{0}\left(x^{\prime}\right)+\varepsilon_{0}\left(x_{k}\right)}{c \varphi_{0}\left(x^{\prime}\right)+\varepsilon_{0}\left(x_{k}\right)} \cdot \frac{c \varphi_{0}\left(x^{\prime}\right)+\varepsilon_{0}\left(x_{k}\right)}{c \varphi_{0}\left(x^{\prime}\right)} \cdot \varepsilon_{0}\left(x^{\prime}\right) \\
& =c^{-1}\left(\varepsilon_{0}\left(x^{\prime}\right)+\frac{\varepsilon_{0}\left(x^{\prime}\right) \varepsilon_{0}\left(x_{k}\right)}{\varphi_{0}\left(x^{\prime}\right)}\right) \\
& =c^{-1} \varepsilon_{0}\left(x_{1}, \cdots, x_{k}\right) .
\end{aligned}
$$

Finally, let us check the Verma relations. We need to see the following cases:

(i) The case $\mathfrak{g}=A_{n}^{(1)} \cdot \begin{cases}e_{0}^{a} e_{i}^{a b} e_{0}^{b}=e_{i}^{b} e_{0}^{a b} e_{i}^{a} & \text { if } i=1, n, \\ e_{0}^{c} e_{i}^{d}=e_{i}^{d} e_{0}^{c} & \text { if } i \neq 1, n .\end{cases}$

(ii) The case $\mathfrak{g}=B_{n}^{(1)}, D_{n}^{(1)}, A_{2 n-1}^{(2)} \cdot \begin{cases}e_{0}^{a} e_{i}^{a b} e_{0}^{b}=e_{i}^{b} e_{0}^{a b} e_{i}^{a} & \text { if } i=2, \\ e_{0}^{c} e_{i}^{d}=e_{i}^{d} e_{0}^{c} & \text { if } i \neq 2 .\end{cases}$

(iii) The case $\mathfrak{g}=D_{n+1}^{(2)}, A_{2 n}^{(2)} \cdot \begin{cases}e_{0}^{a} e_{i}^{a^{2} b} e_{0}^{a b} e_{i}^{b}=e_{i}^{b} e_{0}^{a b} e_{i}^{a^{2} b} e_{0}^{a} & \text { if } i=1, \\ e_{0}^{c} e_{i}^{d}=e_{i}^{d} e_{0}^{c} & \text { if } i \neq 1 .\end{cases}$

By the result in KOTY, we have the product structure on $\left\{\mathcal{B}_{l}\left(A_{n}^{(1)}\right)\right\}$ and $\left\{\mathcal{B}_{l}\left(D_{N}^{(1)}\right)\right\}$. Since $\mathcal{V}\left(A_{n}^{(1)}\right)_{l} \cong \mathcal{B}_{l}\left(A_{n}^{(1)}\right)$ and $\mathcal{V}\left(D_{n}^{(1)}\right)_{l} \cong \mathcal{B}_{l}\left(D_{n}^{(1)}\right)$, we also have the product structure on $\left\{\mathcal{V}\left(A_{n}^{(1)}\right)_{l}\right\}$ and $\left\{\mathcal{V}\left(D_{n}^{(1)}\right)_{l}\right\}$. Hence, we have the Verma relations on their product. Thus, we obtain the case (i) and (ii) $\mathfrak{g}=D_{n}^{(1)}$.

For the case $\sigma(i) \neq 0$, it is easy to check the relation. Indeed, e.g., for the case (iii) $i=1$, we have:

$$
\begin{aligned}
e_{0}^{a} e_{1}^{a^{2} b} e_{0}^{a b} e_{1}^{b} & =\left(\bar{\sigma}^{-1} e_{n}^{a} \bar{\sigma}\right)\left(\bar{\sigma}^{-1} e_{n-1}^{a^{2} b} \bar{\sigma}\right)\left(\bar{\sigma}^{-1} e_{n}^{a b} \bar{\sigma}\right)\left(\bar{\sigma}^{-1} e_{n-1}^{b} \bar{\sigma}\right) \\
& =\bar{\sigma}^{-1}\left(e_{n}^{a} e_{n-1}^{a^{2} b} e_{n}^{a b} e_{n-1}^{b}\right) \bar{\sigma}=\bar{\sigma}^{-1}\left(e_{n-1}^{b} e_{n}^{a b} e_{n-1}^{a^{2} b} e_{n}^{a}\right) \bar{\sigma}=e_{1}^{b} e_{0}^{a b} e_{1}^{a^{2} b} e_{0}^{a} .
\end{aligned}
$$

Similarly, the other cases with $\sigma(i) \neq 0$ can be shown.

To complete (ii) and (iii), it suffices to check the cases $\sigma(i)=0$; i.e., (ii) $i=1$, (iii) $i=n$.

In the previous section we see that for $\mathfrak{g} \neq A_{n}^{(1)}$ the geometric crystal $\mathcal{V}(\mathfrak{g})_{l}$ is obtained from the geometric crystal $\mathcal{B}\left(D_{N}^{(1)}\right)_{l^{\prime}}\left(l^{\prime}=l\right.$ or $\left.l^{2}\right)$ by the method of foldings. Thus, in the case (ii) we have $\left(e_{0}^{\mathfrak{g}}\right)^{c}=\left(e_{0}^{D_{N}^{(1)}}\right)^{c}$ and $\left(e_{1}^{\mathfrak{g}}\right)^{c}=\left(e_{1}^{D_{N}^{(1)}}\right)^{c}$ for $\mathfrak{g}=B_{n}^{(1)}, A_{2 n-1}^{(2)}$. Since $\left(e_{0}^{D_{N}^{(1)}}\right)^{c}\left(e_{1}^{D_{N}^{(1)}}\right)^{d}=\left(e_{1}^{D_{N}^{(1)}}\right)^{d}\left(e_{0}^{D_{N}^{(1)}}\right)^{c}$, we have $\left(e_{0}^{\mathfrak{g}}\right)^{c}\left(e_{1}^{\mathfrak{g}}\right)^{d}=$ $\left(e_{1}^{\mathfrak{g}}\right)^{d}\left(e_{0}^{\mathfrak{g}}\right)^{c}$ and then we completed (ii). In the case (iii), we have $\left(e_{0}^{\mathfrak{g}}\right)^{c}=\left(e_{0}^{D_{N}^{(1)}}\right)^{c}$ and

$$
\left(e_{n}^{\mathfrak{g}}\right)^{c}= \begin{cases}\left(e_{n+1}^{D_{n+2}^{(1)}}\right)^{c} \circ\left(e_{n+2}^{D_{n+2}^{(1)}}\right)^{c} & \mathfrak{g}=D_{n+1}^{(2)}, \\ \left(e_{n+1}^{D_{2 n+2}^{(1)}}\right)^{c} & \mathfrak{g}=A_{2 n}^{(2)} .\end{cases}
$$

Then $\left(e_{0}^{\mathfrak{g}}\right)^{c}\left(e_{n}^{\mathfrak{g}}\right)^{d}=\left(e_{n}^{\mathfrak{g}}\right)^{d}\left(e_{0}^{\mathfrak{g}}\right)^{c}$, which completes (iii). 
By Theorem 5.4, Theorem 7.1] and Theorem 7.2, we obtain

Corollary 7.3. For positive real numbers $L_{j}(j=1, \cdots, k)$, let $\theta_{L_{j}}: T^{\prime} \rightarrow \mathcal{V}(\mathfrak{g})_{L_{j}}$ be the positive structure as in the previous section. Then

$$
\Theta:=\left(\theta_{L_{1}}, \cdots, \theta_{L_{k}}\right): T^{\prime \times k} \rightarrow \mathcal{V}(\mathfrak{g})_{L_{1}} \times \cdots \times \mathcal{V}(\mathfrak{g})_{L_{k}}
$$

defines a positive structure on $\mathcal{V}(\mathfrak{g})_{L_{1}} \times \cdots \times \mathcal{V}(\mathfrak{g})_{L_{k}}$ and we have the isomorphism of geometric crystals:

$$
\mathcal{U} D_{\Theta}\left(\mathcal{V}(\mathfrak{g})_{L_{1}} \times \cdots \times \mathcal{V}(\mathfrak{g})_{L_{k}}\right) \cong B_{\infty}\left(\mathfrak{g}^{L}\right)^{\otimes k}
$$

\section{8. $M$-MATRICES AND AUTOMORPHISMS}

8.1. Definition of M-matrices. An M-matrix is an important object to realize tropical R map from geometric crystals, though its exact definition is not yet fixed. In this paper, we take the following as a temporary definition of M-matrices.

Definition 8.1. Let $\mathfrak{g}$ be an affine Lie algebra and $\mathcal{B}_{L} \subset\left(\mathbb{C}^{\times}\right)^{k}$ be a certain $\mathfrak{g}$ geometric crystal depending on $L \in \mathbb{C}^{\times}$with a product structure. For $x \in \mathcal{B}_{L}$ and

an indeterminate $z$, a square matrix $M_{L}(x, z)$ with entries in $\mathbb{C}(x, z)$ is an M-matrix if:

(i) For $x \in \mathcal{B}_{L}, i \in I$ and $c \in \mathbb{C}^{\times}$, there exist non-singular matrices $X_{i}(x, c, z)$ and $Y_{i}(x, c, z)$ whose entries are in $\mathbb{C}(x, c, z)$ and satisfying

$$
M_{L}\left(e_{i}^{c}(x), z\right)=X_{i}(x, c, z) M_{L}(x, z) Y_{i}(x, c, z) .
$$

(ii) For a given $(x, y) \in \mathcal{B}_{L} \times \mathcal{B}_{K}$ and $L, K \in \mathbb{C}^{\times}$, the solution $\left(x^{\prime}, y^{\prime}\right) \in \mathcal{B}_{K} \times \mathcal{B}_{L}$ of the equation

$$
M_{L}(x, z) M_{K}(y, z)=M_{K}\left(x^{\prime}, z\right) M_{L}\left(y^{\prime}, z\right),
$$

uniquely exists for any $z$ and the correspondence $(x, y) \mapsto\left(x^{\prime}, y^{\prime}\right)$ defines a birational map $R: \mathcal{B}_{L} \times \mathcal{B}_{K} \rightarrow \mathcal{B}_{K} \times \mathcal{B}_{M}$.

(iii) Let $x_{j}, y_{j}$ be in $\mathcal{B}_{L_{j}}(j=1,2, \ldots, m)$. Suppose $L_{i} \neq L_{j}(i \neq j)$ and

$$
M_{L_{1}}\left(x_{1}, z\right) \cdots M_{L_{m}}\left(x_{m}, z\right)=M_{L_{1}}\left(y_{1}, z\right) \cdots M_{L_{m}}\left(y_{m}, z\right) .
$$

Then $x_{j}=y_{j}(j=1, \ldots, m)$.

Example 8.2 ([KOTY $]$. $A_{n}^{(1)}$-case: Let $\mathcal{B}_{L}\left(A_{n}^{(1)}\right)$ be the geometric crystal as in Section [5.3. For $l=\left(l_{1}, \ldots, l_{n+1}\right) \in \mathcal{B}_{L}\left(A_{n}^{(1)}\right)$, set

$$
M_{L}(l, z):=\left(\begin{array}{cccccc}
l_{1}^{-1} & & & & & -z \\
-1 & l_{2}^{-1} & & & & \\
& & \ldots & & & \\
& & & -1 & l_{n}^{-1} & \\
& & & & -1 & l_{n+1}^{-1}
\end{array}\right)^{-1} .
$$

Then the matrix $M_{L}(l, z)$ is an M-matrix. 
8.2. Explicit form of the M-matrix of type $D_{n}^{(1)}$. An explicit form of the M-matrix $M_{L}(l, z)$ for $\mathcal{B}_{L}\left(D_{n}^{(1)}\right)$ is described KOTY where the geometric crystal $\mathcal{B}_{L}\left(D_{n}^{(1)}\right)$ is as in Section 6

For $l=\left(l_{1}, \ldots, l_{n}, \bar{l}_{n-1}, \ldots, \bar{l}_{1}\right) \in \mathcal{B}_{L}\left(D_{n}^{(1)}\right)$, the M-matrix $M_{L}(l, z)$ is given as follows [KOTY]: $M(l, z)$ is a $2 n \times 2 n$ matrix in the form $M_{L}(l, z)=A(l)+z B(l)+$ $z^{2} C(l)$ where each matrix $A(l), B(l)$ and $C(l)$ are given by $C(l)=E_{1,2 n}$,

$$
\begin{aligned}
& A(l)_{i, i}= \begin{cases}\frac{l_{i}}{\bar{l}_{i}} & 1 \leq i \leq n-1, \\
l_{n} & i=n, \\
\frac{1}{l_{n}} & i=n+1, \\
\frac{l_{2 n+1-i}}{l_{2 n+1-i}} & n+2 \leq i \leq 2 n,\end{cases} \\
& A(l)_{i, j}=l_{j} \cdots l_{i-1}\left(1+\frac{l_{i}}{\bar{l}_{i}}\right) \quad 1 \leq j<i \leq n-1, \\
& A(l)_{2 n+1-j, 2 n+1-i}=\bar{l}_{j} \cdots \bar{l}_{i-1}\left(1+\frac{\bar{l}_{i}}{l_{i}}\right) \quad 1 \leq j<i \leq n-1, \\
& A(l)_{n, j}=l_{j} \cdots l_{n} \quad 1 \leq j \leq n-1, \\
& A(l)_{2 n+1-j, n}=\bar{l}_{j} \cdots \bar{l}_{n-1} l_{n} \quad 1 \leq j \leq n-1, \\
& A(l)_{2 n+1-j, n+1}=\bar{l}_{j} \cdots \bar{l}_{n-1} \quad 1 \leq j \leq n-1, \\
& A(l)_{2 n+1-i, j}=\left(l_{j} \cdots l_{n}\right)\left(\bar{l}_{i} \cdots \bar{l}_{n-1}\right) \quad 1 \leq i, j \leq n-1, \\
& A(l)_{i, j}=0 \quad \text { otherwise. }
\end{aligned}
$$

The matrix element $B(l)_{i, j}$ is given by $B(l)_{i, j}=A(l)_{i, 1} A(l)_{2 n, j}-L A(l)_{i, j}-\delta_{i, 1} \delta_{j, 2 n}$ ([KOTY] $)$.

8.3. Matrix realization. In terms of M-matrices, the involutions $\Sigma_{0}^{(n)}, \Sigma_{1}^{(n)}, \Sigma_{2}^{(n)}$ in Section [6] are realized by the adjoint action of certain matrices.

Proposition 8.3. For each involution $\Sigma_{i}^{(n)}(i=0,1,2)$, there exists a non-singular matrix $J_{i}^{(n)}=J_{i}^{(n)}(z)$ such that

$$
M_{L}\left(\Sigma_{i}^{(n)}(l), z\right)=J_{i}^{(n)}(z) M_{L}(l, z) J_{i}^{(n)}(z)^{-1},
$$

where the matrix $J_{i}^{(n)}$ does not depend on $l$. Each $J_{i}^{(n)}$ is as follows:

$$
\begin{gathered}
J_{0}^{(n)}=\left(\begin{array}{c|c|c}
0 & 0 & z \\
\hline 0 & E_{2 n} & 0 \\
\hline z^{-1} & 0 & 0
\end{array}\right), \quad J_{1}^{(n)}=\left(\begin{array}{c|c|c|c}
E_{n} & 0 & 0 \\
\hline 0 & 0 & 1 & 0 \\
& 1 & 0 & 0 \\
\hline 0 & 0 & E_{n}
\end{array}\right), \\
J_{2}^{(n)}=\left(\begin{array}{c|c}
0 & z \cdot E_{2 n} \\
\hline E_{2 n} & 0
\end{array}\right),
\end{gathered}
$$

where $E_{m}$ is the identity matrix of size $m$. The size of each matrix $J_{i}^{(n)}$ and $M_{L}(l, z)$ is $2 n+2$ for $i=0$ and $i=1$, and $4 n$ for $i=2$. Setting $J_{3}^{(n)}=J_{0}^{(n+1)} J_{1}^{(n+1)}$ $\left(=J_{1}^{(n+1)} J_{0}^{(n+1)}\right)$ and $J_{4}^{(n)}=\left(J_{0}^{(2 n+1)} J_{1}^{(2 n+1)}\right) J_{2}^{(n+1)}\left(=J_{2}^{(n+1)}\left(J_{0}^{(2 n+1)} J_{1}^{(2 n+1)}\right)\right)$, we also have

$$
M_{L}\left(\Sigma_{k}^{(n)}(l), z\right)=J_{k}^{(n)} M_{L}(l, z) J_{k}^{(n)^{-1}} \quad(k=3,4) .
$$


Proof. Since the involution $\Sigma_{0}^{(n)}$ (resp. $\Sigma_{1}^{(n)}$ ) coincides with the involution $\sigma_{1}$ (resp. $\sigma_{n}$ ) in [KOTY and the matrix $J_{0}^{(n)}$ (resp. $J_{1}^{(n)}$ ) is identified with the matrix $J_{1}(z)$ (resp. $\left.J_{n}(z)\right)$ as in KOTY. Thus, Lemma 3.10 in [KOTY] shows that our assertions for $\Sigma_{k}^{(n)}(k=0,1)$ are right. Then let us show the case $k=2$. Since we have the explicit form of $M_{L}(l, z)$ as in the last subsection, it is carried out by case-by-case calculations. For example, let us see the diagonal entries:

$$
M_{L}\left(\Sigma_{2}^{(n)}(l), z\right)_{i, i}= \begin{cases}1 / l_{2 n} & i=1, \\ \frac{\bar{l}_{2 n+1-i}}{l_{2 n+1-i}}+z L & 2 \leq i \leq 2 n-1, \\ \bar{l}_{1} / l_{1} & i=2 n, \\ l_{1} / \bar{l}_{1} & i=2 n+1, \\ \frac{l_{i-2 n}}{\bar{l}_{i-2 n}}+z L & 2 n+2 \leq i \leq 4 n-1, \\ l_{2 n} & i=4 n .\end{cases}
$$

Here note that the matrix $M_{L}(l, z)$ is a $4 n \times 4 n$-matrix.

On the other hand, the diagonal entries of the matrix

$$
M^{\prime}:=J_{n}^{(2)} M_{L}(l, z) J_{n}^{(2)^{-1}}=J_{n}^{(2)}\left(\begin{array}{ll}
M_{1} & M_{2} \\
M_{3} & M_{4}
\end{array}\right) J_{n}^{(2)^{-1}}=\left(\begin{array}{cc}
M_{4} & z M_{3} \\
z^{-1} M_{2} & M_{1}
\end{array}\right)
$$

are given by:

For $i=1, \ldots, 2 n$,

$$
M_{i, i}^{\prime}=M_{L}(l, z)_{i+2 n, i+2 n}= \begin{cases}1 / l_{2 n} & i=1, \\ \bar{l}_{2 n+1-i} & \bar{l}_{2 n+1-i} \\ \bar{l}_{1} / l_{1} L & 2 \leq i \leq 2 n-1, \\ & i=2 n\end{cases}
$$

For $i=2 n+1, \ldots, 4 n$,

$$
M_{i, i}^{\prime}=M_{L}(l, z)_{i-2 n, i-2 n}= \begin{cases}l_{1} / \bar{l}_{1} & i=2 n+1, \\ l_{i-2 n}+z L & 2 n+2 \leq i \leq 4 n-1, \\ \bar{l}_{i-2 n} & i=4 n .\end{cases}
$$

Then, we have $M_{L}\left(\Sigma_{n}^{(2)}(l), z\right)_{i, i}=M_{i, i}^{\prime}\left(=\left(J_{n}^{(2)} M_{L}(l, z) J_{n}^{(2)^{-1}}\right)_{i, i}\right)$. The other cases are also obtained similarly.

8.4. Birational maps on fixed point varieties. Let $\mathcal{R}_{L K}$ be the birational map defined by

$$
\begin{aligned}
\mathcal{R}_{L K}: \mathcal{B}_{L}\left(D_{N}^{(1)}\right) \times \mathcal{B}_{K}\left(D_{N}^{(1)}\right) & \rightarrow \mathcal{B}_{K}\left(D_{N}^{(1)}\right) \times \mathcal{B}_{L}\left(D_{N}^{(1)}\right)\left(L, K \in \mathbb{C}^{\times}\right) \\
(l, m) & \mapsto\left(l^{\prime}, m^{\prime}\right),
\end{aligned}
$$

where $\left(l^{\prime}, m^{\prime}\right)$ be the unique solution of the equation

$$
M_{L}(l, z) M_{K}(m, z)=M_{K}\left(l^{\prime}, z\right) M_{L}\left(m^{\prime}, z\right) .
$$

Let $X_{i}^{(n, L)}$ be one of the fixed point subvarieties in $\mathcal{B}_{L}\left(D_{N}^{(1)}\right)$.

Theorem 8.4. Let us denote $\mathcal{R}_{L K}^{(i)}$ as the restriction of $\mathcal{R}_{L K}$ on $X_{i}^{(n, L)} \times X_{i}^{(n, K)}$. Then $\mathcal{R}_{L K}^{(i)}$ is a well-defined birational map $X_{i}^{(n, L)} \times X_{i}^{(n, K)} \rightarrow X_{i}^{(n, K)} \times X_{i}^{(n, L)}$ $\left(L, K \in \mathbb{C}^{\times}\right)$. 
Proof. For $(l, m) \in X_{i}^{(n, L)} \times X_{i}^{(n, K)}$, set $\left(l^{\prime}, m^{\prime}\right):=\mathcal{R}(l, m)$, i.e.,

$$
M_{L}(l, z) M_{K}(m, z)=M_{K}\left(l^{\prime}, z\right) M_{L}\left(m^{\prime}, z\right) .
$$

We have

$$
\begin{aligned}
M_{L}(l, z) M_{K}(m, z) & =M_{L}\left(\Sigma_{i}^{(n)}(l), z\right) M_{K}\left(\Sigma_{i}^{(n)}(m), z\right) \\
& =J_{i}^{(n)} M_{L}(l, z) M_{K}(m, z) J_{i}^{(n)^{-1}} \\
& =J_{i}^{(n)} M_{K}\left(l^{\prime}, z\right) M_{L}\left(m^{\prime}, z\right) J_{i}^{(n)^{-1}} \\
& =M_{K}\left(\Sigma_{i}^{(n)}\left(l^{\prime}\right), z\right) M_{L}\left(\Sigma_{i}^{(n)}\left(m^{\prime}\right), z\right) .
\end{aligned}
$$

Then, we have

$$
M_{K}\left(l^{\prime}, z\right) M_{L}\left(m^{\prime}, z\right)=M_{K}\left(\Sigma_{i}^{(n)}\left(l^{\prime}\right), z\right) M_{L}\left(\Sigma_{i}^{(n)}\left(m^{\prime}\right), z\right) .
$$

It follows from the uniqueness of the solution for 8.10 that

$$
\left(l^{\prime}, m^{\prime}\right)=\left(\Sigma_{i}^{(n)}\left(l^{\prime}\right), \Sigma_{i}^{(n)}\left(m^{\prime}\right)\right)
$$

and then we have $\left(l^{\prime}, m^{\prime}\right) \in X_{i}^{(n, K)} \times X_{i}^{(n, L)}$.

\section{TROpical R MAPS}

In this section, we define the notion of tropical $\mathrm{R}$ map and give explicit forms of the affine tropical $\mathrm{R}$ maps on the geometric crystals constructed above.

\subsection{Definition of tropical $R$ map.}

Definition 9.1. Let $\left\{\left(X_{\lambda},\left\{e_{i}^{\lambda}\right\},\left\{\gamma_{i}^{\lambda}\right\},\left\{\varepsilon_{i}^{\lambda}\right\}\right)\right\}_{\lambda \in \Lambda}$ be a family of geometric crystals equipped with the product structures, where $\Lambda$ is a certain index set and its element is called a spectral parameter. A birational map $\mathcal{R}_{\lambda \mu}: X_{\lambda} \times X_{\mu} \longrightarrow X_{\mu} \times X_{\lambda}$ $(\lambda, \mu \in \Lambda)$ is said to be a tropical $R$ map (or shortly, tropical $\mathrm{R}$ ) if it satisfies the following conditions:

$$
\begin{aligned}
& \left(e_{i}^{X_{\mu} \times X_{\lambda}}\right)^{c} \circ \mathcal{R}_{\lambda \mu}=\mathcal{R}_{\lambda \mu} \circ\left(e_{i}^{X_{\lambda} \times X_{\mu}}\right)^{c}, \\
& \varepsilon_{i}^{X_{\lambda} \times X_{\mu}}=\varepsilon_{i}^{X_{\mu} \times X_{\lambda}} \circ \mathcal{R}_{\lambda \mu}, \\
& \gamma_{i}^{X_{\lambda} \times X_{\mu}}=\gamma_{i}^{X_{\mu} \times X_{\lambda}} \circ \mathcal{R}_{\lambda \mu}, \\
& \mathcal{R}^{(12)} \mathcal{R}^{(23)} \mathcal{R}^{(12)}=\mathcal{R}^{(23)} \mathcal{R}^{(12)} \mathcal{R}^{(23)} \quad \text { on } X_{\lambda} \times X_{\mu} \times X_{\nu}, \\
& \mathcal{R}_{\mu \lambda} \mathcal{R}_{\lambda \mu}=\mathrm{id}_{\lambda \mu},
\end{aligned}
$$

for any $i \in I$ and any $\lambda, \mu, \nu \in \Lambda$. Here $\mathcal{R}^{(i j)}$ means that it acts on $i$-th and $j$-th components of the product.

In the rest of this section, we give the explicit forms of the tropical $\mathcal{R}$ for the affine geometric crystals.

9.2. $D_{n}^{(1)}$ case $(n \geq 4)$. Let $\mathcal{R}_{L K}: \mathcal{B}_{L}\left(D_{N}^{(1)}\right) \times \mathcal{B}_{K}\left(D_{N}^{(1)}\right) \rightarrow \mathcal{B}_{K}\left(D_{N}^{(1)}\right) \times \mathcal{B}_{L}\left(D_{N}^{(1)}\right)$ $\left(L, K \in \mathbb{C}^{\times}\right)$be the birational map as in 8.4. In [KOTY], it is shown that the morphism $\mathcal{R}_{L M}$ is a tropical $\mathrm{R}$ map for $\left\{\mathcal{B}_{L}\left(D_{n}^{(1)}\right)\right\}_{L \in \mathbb{C}^{\times}}$. We shall describe the explicit form of $\mathcal{R}_{L M}$. Let $\sharp: \mathcal{B}_{L}\left(D_{n}^{(1)}\right) \rightarrow \mathcal{B}_{L}\left(D_{n}^{(1)}\right)$ be an involution defined by

$$
\sharp\left(l_{1}, l_{2}, \ldots, l_{n}, \bar{l}_{n-1}, \ldots, \bar{l}_{2}, \bar{l}_{1}\right)=\left(\bar{l}_{1}, l_{2}, \ldots, l_{n}, \bar{l}_{n-1}, \ldots, \bar{l}_{2}, l_{1}\right),
$$


that is, $\sharp: l_{1} \leftrightarrow \bar{l}_{1}$ and $*: \mathcal{B}_{L}\left(D_{n}^{(1)}\right) \times \mathcal{B}_{M}\left(D_{n}^{(1)}\right) \rightarrow \mathcal{B}_{M}\left(D_{n}^{(1)}\right) \times \mathcal{B}_{L}\left(D_{n}^{(1)}\right)$ an involution defined by

$\left(\left(l_{1}, l_{2}, \ldots, \bar{l}_{2}, \bar{l}_{1}\right),\left(m_{1}, m_{2}, \ldots, \bar{m}_{2}, \bar{m}_{1}\right)\right)^{*}=\left(\left(\bar{m}_{1}, \bar{m}_{2}, \ldots, m_{2}, m_{1}\right),\left(\bar{l}_{1}, \bar{l}_{2}, \ldots, l_{2}, l_{1}\right)\right)$

that is, $*: l_{i} \leftrightarrow \bar{m}_{i}, \quad \bar{l}_{i} \leftrightarrow m_{i}(1 \leq i \leq n-1), \quad l_{n} \leftrightarrow m_{n}$.

Following [KOTY], we define the rational functions $V_{i}(i=0,1, \ldots, n-1)$ and $W_{i}(i=1, \ldots, n-1)$ on $\mathcal{B}_{L}\left(D_{n}^{(1)}\right) \times \mathcal{B}_{M}\left(D_{n}^{(1)}\right)\left(L, M \in \mathbb{C}^{\times}\right)$by

$W_{i}:=V_{i} V_{i}^{*}+(M-L) V_{i}^{*}+(L-M) V_{i} \quad(1 \leq i \leq n-2), \quad W_{n-1}:=V_{n-1} V_{n-1}{ }^{*}$.

$$
V_{i}=\sum_{j=1}^{n-2}\left(\theta_{i, j}(l, m)+\theta_{i, j}^{\prime}(l, m)\right)+\sum_{j=1}^{n}\left(\eta_{i, j}(l, m)+\eta_{i, j}^{\prime}(l, m)\right)
$$

where we set $L=l_{1} l_{2} \cdots \bar{l}_{2} \bar{l}_{1}, M=m_{1} m_{2} \cdots \bar{m}_{2} \bar{m}_{1}$,

$$
\eta_{i, j}(l, m)= \begin{cases}L\left(\prod_{k=j+1}^{i} \frac{\bar{m}_{k}}{\bar{l}_{k}}\right)\left(\frac{\bar{m}_{j}}{l_{j}}\right) & \text { for } \quad 1 \leq j \leq i, \\ M\left(\prod_{k=i+1}^{j} \frac{\bar{l}_{k}}{\bar{m}_{k}}\right)\left(\frac{\bar{m}_{j}}{l_{j}}\right) & \text { for } \quad i+1 \leq j \leq n-1, \\ M\left(\prod_{k=i+1}^{n-1} \frac{\bar{l}_{k}}{\bar{m}_{k}}\right) l_{n} & \text { for } \quad j=n,\end{cases}
$$

$$
\eta_{i, j}^{\prime}(l, m)= \begin{cases}L\left(\prod_{k=1}^{i} \frac{\bar{m}_{k}}{\bar{l}_{k}}\right)\left(\prod_{k=1}^{j} \frac{m_{k}}{l_{k}}\right)\left(\frac{l_{j}}{\bar{m}_{j}}\right) & \text { for } \quad 1 \leq j \leq n-1, \\ L\left(\frac{L}{M}\right)^{\delta_{i, n-1}}\left(\prod_{k=1}^{i} \frac{\bar{m}_{k}}{\bar{l}_{k}}\right)\left(\prod_{k=1}^{n-1} \frac{m_{k}}{l_{k}}\right)\left(\frac{1}{l_{n}}\right) & \text { for } \quad j=n .\end{cases}
$$

Remark. Though $W_{i}$ seems not to be a positive rational function, it is, indeed, positive by the formula (KOTY Lemma 4.14):

$$
\left(\frac{1}{l_{i}}+\frac{1}{\bar{m}_{i}}\right) W_{i}=\frac{1}{m_{i}} V_{i} V_{i-1}^{*}+\frac{1}{\bar{l}_{i}} V_{i-1} V_{i}^{*} .
$$


Now, we introduce the following rational transformation $\mathcal{R}$ on $\left(\mathbb{C}^{\times}\right)^{2 n-1} \times\left(\mathbb{C}^{\times}\right)^{2 n-1}$ :

$$
\mathcal{R}(l, m)=\left(l^{\prime}, m^{\prime}\right)
$$

where

$$
\begin{aligned}
& l_{1}^{\prime}=m_{1} \frac{V_{0}^{\sharp}}{V_{1}}, \quad \bar{l}_{1}^{\prime}=\bar{m}_{1} \frac{V_{0}}{V_{1}}, \quad l_{i}^{\prime}=m_{i} \frac{V_{i-1} W_{i}}{V_{i} W_{i-1}}, \quad \bar{l}_{i}^{\prime}=\bar{m}_{i} \frac{V_{i-1}}{V_{i}} \quad(2 \leq i \leq n-1), \\
& l_{n}^{\prime}=m_{n} \frac{V_{n-1}}{V_{n-1}^{*}}, \quad m_{1}^{\prime}=l_{1} \frac{V_{0}}{V_{1}^{*}}, \quad \bar{m}_{1}^{\prime}=\bar{l}_{1} \frac{V_{0}^{\sharp}}{V_{1}^{*}}, \\
& m_{i}^{\prime}=l_{i} \frac{V_{i-1}^{*}}{V_{i}^{*}}, \quad \bar{m}_{i}^{\prime}=\bar{l}_{i} \frac{V_{i-1}^{*} W_{i}}{V_{i}^{*} W_{i-1}} \quad(2 \leq i \leq n-1), \quad m_{n}^{\prime}=l_{n} \frac{V_{n-1}^{*}}{V_{n-1}} .
\end{aligned}
$$

Here note that for $\left(l^{\prime}, m^{\prime}\right)=\mathcal{R}(l, m)$ we have $l_{1}^{\prime} l_{2}^{\prime} \cdots \bar{l}_{2}^{\prime} \bar{l}_{1}^{\prime}=M$ and $m_{1}^{\prime} m_{2}^{\prime} \cdots \bar{m}_{2}^{\prime} \bar{m}_{1}^{\prime}=$ $L$. Then $\mathcal{R}$ defines a rational map $\mathcal{B}_{L}\left(D_{n}^{(1)}\right) \times \mathcal{B}_{M}\left(D_{n}^{(1)}\right) \longrightarrow \mathcal{B}_{M}\left(D_{n}^{(1)}\right) \times \mathcal{B}_{L}\left(D_{n}^{(1)}\right)$. The following is one of the main results in $\mathrm{KOTY}$ :

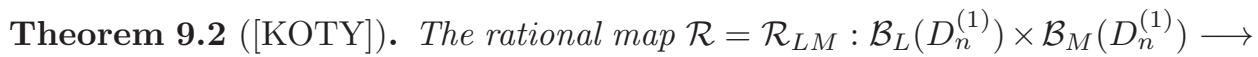
$\mathcal{B}_{M}\left(D_{n}^{(1)}\right) \times \mathcal{B}_{L}\left(D_{n}^{(1)}\right)$ gives a tropical $R$ map of type $D_{n}^{(1)}$ on the family of affine geometric crystals, which will be denoted by $\mathcal{R}\left(D_{n}^{(1)}\right)$ in the sequel.

Here we describe the tropical R on $\mathcal{V}\left(D_{n}^{(1)}\right)_{L} \times \mathcal{V}\left(D_{n}^{(1)}\right)_{M}: \overline{\mathcal{R}}(x, y):=(\Xi, \Xi) \circ \mathcal{R} \circ$ $\left(\Xi^{-1}, \Xi^{-1}\right)(x, y)\left(x \in \mathcal{V}\left(D_{n}^{(1)}\right)_{L}, y \in \mathcal{V}\left(D_{n}^{(1)}\right)_{M}\right)$. We define the rational functions $\bar{V}_{i}(i=0,1, \ldots, n-1)$ and $\bar{W}_{i}(i=1, \ldots, n-1)$ on $\mathcal{V}\left(D_{n}^{(1)}\right)_{L} \times \mathcal{V}\left(D_{n}^{(1)}\right)_{M}(L, M \in$ $\left.\mathbb{C}^{\times}\right)$by

$\bar{W}_{i}:=\bar{V}_{i} \bar{V}_{i}^{*}+(M-L) \bar{V}_{i}^{*}+(L-M) \bar{V}_{i} \quad(1 \leq i \leq n-2), \quad \bar{W}_{n-1}:=\bar{V}_{n-1} \bar{V}_{n-1}^{*}$.

$$
\bar{V}_{i}=\sum_{j=1}^{n-2}\left(\bar{\theta}_{i, j}(x, y)+\bar{\theta}_{i, j}^{\prime}(x, y)\right)+\sum_{j=1}^{n}\left(\bar{\eta}_{i, j}(x, y)+\bar{\eta}_{i, j}^{\prime}(x, y)\right) \text {, }
$$

where $\sharp$ is the involution on $\mathcal{V}\left(D_{n}^{(1)}\right)_{L}$ and $*$ is the involution $\mathcal{V}\left(D_{n}^{(1)}\right)_{L} \times \mathcal{V}\left(D_{n}^{(1)}\right)_{M} \rightarrow$ $\mathcal{V}\left(D_{n}^{(1)}\right)_{M} \times \mathcal{V}\left(D_{n}^{(1)}\right)_{L}$ defined by

$$
\begin{aligned}
\# \quad: \quad x_{i} \mapsto \frac{x_{i}}{L x_{1} \bar{x}_{1}} \quad(i=1 \cdots, n) \quad, \bar{x}_{i} \mapsto \frac{\bar{x}_{i}}{L x_{1} \bar{x}_{1}} \quad(i=1, \cdots, n-2), \\
* \quad: \quad x_{i} \mapsto \frac{1}{M \bar{y}_{i}}, \quad \bar{x}_{i} \mapsto \frac{1}{M y_{i}} \quad(i=1, \cdots, n-2), \\
x_{n-1} \mapsto \frac{1}{M y_{n}}, \quad x_{n} \mapsto \frac{1}{M y_{n-1}}, \\
y_{i} \mapsto \frac{1}{L \bar{x}_{i}}, \quad \bar{y}_{i} \mapsto \frac{1}{L x_{i}} \quad(i=1, \cdots, n-2), \quad y_{n-1} \mapsto \frac{1}{L x_{n}}, \quad y_{n} \mapsto \frac{1}{L x_{n-1}},
\end{aligned}
$$


and

$$
\begin{aligned}
& \bar{\theta}_{i, j}(x, y):=L \frac{x_{i} y_{j}}{x_{j} y_{i}} \quad(1 \leq j \leq i<n-1), \\
& \bar{\theta}_{n-1, j}(x, y):=L \frac{x_{n} y_{j}}{x_{j} y_{n}} \quad(1 \leq j<n-1), \\
& \bar{\theta}_{i, j}(x, y):=M \frac{x_{i} y_{j}}{x_{j} y_{i}} \quad(i+1 \leq j \leq n-2), \\
& \bar{\theta}_{i, j}^{\prime}(x, y):=M \frac{x_{i} \bar{y}_{j}}{\bar{x}_{j} y_{i}}(0 \leq i \leq n-2), \bar{\theta}_{n-1, j}^{\prime}(x, y):=M \frac{x_{n} \bar{y}_{j}}{\bar{x}_{j} y_{n}}, \\
& \bar{\eta}_{i, j}(x, y):=L^{1-\delta_{j, 1}} \frac{x_{i} \bar{x}_{j-1} y_{j-1}}{x_{j} \bar{x}_{j} y_{i}} \quad(1 \leq j \leq i<n-1), \\
& \bar{\eta}_{n-1, j}(x, y):=L^{1-\delta_{j, 1}} \frac{x_{n} \bar{x}_{j-1} y_{j-1}}{x_{j} \bar{x}_{j} y_{n}} \quad(1 \leq j \leq n-1), \\
& \bar{\eta}_{i, j}(x, y):=\frac{M}{L^{\delta_{j, 1}}} \frac{x_{i} \bar{x}_{j-1} y_{j-1}}{x_{j} \bar{x}_{j} y_{i}} \quad(0 \leq i<j \leq n-2), \\
& \bar{\eta}_{i, n-1}(x, y)=M \frac{x_{i} \bar{x}_{n-2} y_{n-2}}{x_{n-1} x_{n} y_{i}} \quad(i+1 \leq n-1), \\
& \bar{\eta}_{i, n}(x, y):=M \frac{x_{i} y_{n}}{x_{n-1} y_{i}} \quad(i \leq n-2), \quad \bar{\eta}_{n-1, n}(x, y):=M \frac{x_{n}}{x_{n-1}}, \\
& \bar{\eta}_{i, j}^{\prime}(x, y):=L^{\delta_{j, 1}} M \frac{x_{i} y_{j} \bar{y}_{j}}{\bar{x}_{j-1} y_{j-1} y_{i}} \quad(1 \leq j \leq n-2, i<n-1), \\
& \bar{\eta}_{n-1, j}^{\prime}(x, y):=L^{\delta_{j, 1}} M \frac{x_{n} y_{j} \bar{y}_{j}}{\bar{x}_{j-1} y_{j-1} y_{n}} \quad(1 \leq j \leq n-2), \\
& \bar{\eta}_{i, n-1}^{\prime}(x, y):=M \frac{x_{i} y_{n-1} y_{n}}{\bar{x}_{n-2} y_{n-2} y_{i}} \quad(0 \leq i<n-1), \quad \bar{\eta}_{n-1, n-1}^{\prime}(x, y):=M \frac{x_{n} y_{n-1}}{\bar{x}_{n-2} y_{n-2}}, \\
& \bar{\eta}_{i, n}^{\prime}(x, y):=M \frac{x_{i} y_{n-1}}{y_{i} x_{n}} \quad(0 \leq i<n-1), \quad \bar{\eta}_{n-1, n}^{\prime}(x, y):=L \frac{y_{n-1}}{y_{n}},
\end{aligned}
$$

where we understand $x_{0}=y_{0}=\bar{x}_{0}=\bar{y}_{0}=1$. Note that in the rest of this section if we write $\bar{\theta}_{i j}=\bar{\theta}_{i, j}^{L M}, \bar{\theta}_{i j}^{\prime}=\bar{\theta}_{i, j}^{L M}, \bar{\eta}_{i j}=\bar{\eta}_{i, j}^{L M}$ and $\bar{\eta}_{i j}^{\prime}=\bar{\eta}_{i, j}^{L M}$, we understand that $\bar{\theta}_{i, j}^{L M}(x, y)^{*}=\bar{\theta}_{i, j}^{M L}\left((x, y)^{*}\right),{\bar{\theta}^{\prime}}_{i, j}^{L M}(x, y)^{*}=\bar{\theta}_{i, j}^{M L}\left((x, y)^{*}\right), \bar{\eta}_{i, j}^{L M}(x, y)^{*}=$ $\bar{\eta}_{i, j}^{M L}\left((x, y)^{*}\right)$ and $\bar{\eta}_{i, j}^{L M}(x, y)^{*}=\bar{\eta}_{i, j}^{\prime M L}\left((x, y)^{*}\right)$.

Set $\overline{\mathcal{R}}=\overline{\mathcal{R}}\left(D_{n}^{(1)}\right): \overline{\mathcal{R}}(x, y)=\left(x^{\prime}, y^{\prime}\right)$ where

$$
\begin{aligned}
& x_{i}^{\prime}:=y_{i} \frac{\bar{V}_{i}}{\bar{V}_{0}}, \quad \bar{x}_{i}^{\prime}:=\bar{y}_{i} \frac{\bar{V}_{0}^{\sharp} \bar{W}_{i}}{\bar{V}_{i} \bar{W}_{1}},(1 \leq i \leq n-2) \\
& x_{n-1}^{\prime}:=y_{n-1} \frac{\bar{V}_{n-1}^{*}}{\bar{V}_{0}}, \quad x_{n}^{\prime}:=y_{n} \frac{\bar{V}_{n-1}}{\bar{V}_{0}}, \\
& y_{i}^{\prime}:=x_{i} \frac{\bar{V}_{i}^{*} \bar{W}_{1}}{\bar{V}_{0}^{\sharp} \bar{W}_{i}}, \quad \bar{y}_{i}^{\prime}:=\bar{x}_{i} \frac{\bar{V}_{0}}{\bar{V}_{i}^{*}}(1 \leq i \leq n-2), \\
& y_{n-1}^{\prime}:=x_{n-1} \frac{\bar{V}_{n-1} \bar{W}_{1}}{\bar{V}_{0}^{\sharp} \bar{W}_{n-1}}, y_{n}^{\prime}:=x_{n} \frac{\bar{V}_{n-1}^{*} \bar{W}_{1}}{\bar{V}_{0}^{\sharp} \bar{W}_{n-1}} .
\end{aligned}
$$


9.3. Tropical $R$ for $B_{n}^{(1)}(n \geq 2)$. By applying the method of folding, we shall obtain an explicit form of the tropical $\mathrm{R}$ for $\left\{\mathcal{B}_{L}\left(B_{n}^{(1)}\right)\right\}_{L \in \mathbb{C} \times}$ and $\left\{\mathcal{V}\left(B_{n}^{(1)}\right)_{L}\right\}_{L \in \mathbb{C} \times}$. Indeed, it follows from Theorem 8.4 that we have the birational map $R\left(B_{n}^{(1)}\right)=$ $R_{L K}\left(B_{n}^{(1)}\right): X_{1}^{(n, L)} \times X_{1}^{(n, K)} \rightarrow X_{1}^{(n, K)} \times X_{1}^{(n, L)}$. Let us see that $R_{L K}\left(B_{n}^{(1)}\right)$ is a tropical R.

Lemma 9.3. The birational map $R\left(B_{n}^{(1)}\right)$ is a tropical $R$ on the $B_{n}^{(1)}$-geometric crystals $\left\{X_{1}^{(n, L)}\right\}_{L}$.

Proof. It suffices to show (9.1)-9.5) in Definition 9.1. The relations (9.4) and (9.5) for $\mathcal{R}\left(B_{n}^{(1)}\right)$ are obtained from the one for $\mathcal{R}\left(D_{n+1}^{(1)}\right)$. The others are easily shown since $\mathcal{R}\left(D_{n+1}^{(1)}\right)$ commutes with the actions of $e_{i}^{c}$ and preserves $\gamma_{i}$ and $\varepsilon_{i}(i \in I)$, and the data $\left(e_{i}^{B_{n}^{(1)}}\right)^{c}, \gamma_{i}^{B_{n}^{(1)}}$ and $\varepsilon_{i}^{B_{n}^{(1)}}$ on $X_{1}^{((n, L))}$ are defined as in Proposition 6.3.

Now let us describe the explicit form of tropical R on $\mathcal{B}_{L}\left(B_{n}^{(1)}\right)$. Set $\mathcal{R}\left(B_{n}^{(1)}\right):=$ $\left(\eta^{-1}, \eta^{-1}\right) \circ R\left(B_{n}^{(1)}\right) \circ(\eta, \eta)$ where $\eta$ is as in 6.3. Let $\sharp: \mathcal{B}_{L}\left(B_{n}^{(1)}\right) \rightarrow \mathcal{B}_{L}\left(B_{n}^{(1)}\right)$ be an involution defined by

$$
\sharp\left(l_{1}, l_{2}, \ldots, l_{n}, \bar{l}_{n}, \ldots, \bar{l}_{2}, \bar{l}_{1}\right)=\left(\bar{l}_{1}, l_{2}, \ldots, l_{n}, \bar{l}_{n}, \ldots, \bar{l}_{2}, l_{1}\right),
$$

that is, $\sharp: l_{1} \leftrightarrow \bar{l}_{1}$. Let $*: \mathcal{B}_{L}\left(B_{n}^{(1)}\right) \times \mathcal{B}_{M}\left(B_{n}^{(1)}\right) \rightarrow \mathcal{B}_{M}\left(B_{n}^{(1)}\right) \times \mathcal{B}_{L}\left(B_{n}^{(1)}\right)$ be an involution defined by

$$
\begin{aligned}
& \quad\left(\left(l_{1}, l_{2}, \ldots, \bar{l}_{2}, \bar{l}_{1}\right),\left(m_{1}, m_{2}, \ldots, \bar{m}_{2}, \bar{m}_{1}\right)\right)^{*} \\
& \quad=\left(\left(\bar{m}_{1}, \bar{m}_{2}, \ldots, m_{2}, m_{1}\right),\left(\bar{l}_{1}, \bar{l}_{2}, \ldots, l_{2}, l_{1}\right)\right),
\end{aligned}
$$

that is, $*: l_{i} \leftrightarrow \bar{m}_{i}, \quad \bar{l}_{i} \leftrightarrow m_{i}(1 \leq i \leq n)$.

Restricting the functions $V_{i}$ and $W_{i}$ for $D_{n+1}^{(1)}$ to $l_{n+1}=m_{n+1}=1$, we define the rational functions $V_{i}(i=0,1, \ldots, n)$ and $W_{i}(i=1, \ldots, n)$ on $\mathcal{B}_{L}\left(B_{n}^{(1)}\right) \times \mathcal{B}_{M}\left(B_{n}^{(1)}\right)$ $\left(L, M \in \mathbb{C}^{\times}\right)$as

$$
\begin{gathered}
W_{i}:=V_{i} V_{i}^{*}+(M-L) V_{i}^{*}+(L-M) V_{i} \quad(1 \leq i \leq n-1), \quad W_{n}:=V_{n} V_{n}^{*}, \\
V_{i}=\sum_{j=1}^{n-1}\left(\theta_{i, j}(l, m)+\theta_{i, j}^{\prime}(l, m)\right)+\sum_{j=1}^{n+1}\left(\eta_{i, j}(l, m)+\eta_{i, j}^{\prime}(l, m)\right),
\end{gathered}
$$


where $L=l_{1} l_{2} \cdots \bar{l}_{2} \bar{l}_{1}, M=m_{1} m_{2} \cdots \bar{m}_{2} \bar{m}_{1}$,

$$
\begin{aligned}
& \theta_{i, j}(l, m)=\left\{\begin{array}{l}
L \prod_{k=j+1}^{i} \frac{\bar{m}_{k}}{\bar{l}_{k}} \quad \text { for } 1 \leq j \leq i, \\
M \prod_{k=i+1}^{j} \frac{\bar{l}_{k}}{\bar{m}_{k}} \quad \text { for } i+1 \leq j \leq n-1,
\end{array}\right. \\
& \theta_{i, j}^{\prime}(l, m)=L\left(\prod_{k=1}^{i} \frac{\bar{m}_{k}}{\bar{l}_{k}}\right)\left(\prod_{k=1}^{j} \frac{m_{k}}{l_{k}}\right) \quad \text { for } \quad j=1, \ldots, n-1, \\
& \eta_{i, j}(l, m)=\left\{\begin{array}{l}
L\left(\prod_{k=j+1}^{i} \frac{\bar{m}_{k}}{\bar{l}_{k}}\right)\left(\frac{\bar{m}_{j}}{l_{j}}\right) \quad \text { for } \quad 1 \leq j \leq i, \\
M\left(\prod_{k=i+1}^{j} \frac{\bar{l}_{k}}{\bar{m}_{k}}\right)\left(\frac{\bar{m}_{j}}{l_{j}}\right) \quad \text { for } \quad i+1 \leq j \leq n, \\
M\left(\prod_{k=i+1}^{n} \frac{\bar{l}_{k}}{\bar{m}_{k}}\right)
\end{array}\right. \\
& \eta_{i, j}^{\prime}(l, m)=\left\{\begin{array}{l}
L\left(\prod_{k=1}^{i} \frac{\bar{m}_{k}}{\bar{l}_{k}}\right)\left(\prod_{k=1}^{j} \frac{m_{k}}{l_{k}}\right)\left(\frac{l_{j}}{\bar{m}_{j}}\right) \quad \text { for } \quad j=n+1, \\
L\left(\frac{L}{M}\right)^{\delta_{i, n}}\left(\prod_{k=1}^{i} \frac{\bar{m}_{k}}{\bar{l}_{k}}\right)\left(\prod_{k=1}^{n} \frac{m_{k}}{l_{k}}\right) \quad \text { for } \quad j=n+1 .
\end{array}\right.
\end{aligned}
$$

Now, we define the tropical R map $\mathcal{R}\left(B_{n}^{(1)}\right)$ on $\mathcal{B}_{L}\left(B_{n}^{(1)}\right) \times \mathcal{B}_{M}\left(B_{n}^{(1)}\right)$ by

$$
\mathcal{R}\left(B_{n}^{(1)}\right)(l, m)=\left(l^{\prime}, m^{\prime}\right)
$$

where

$$
\begin{aligned}
& l_{1}^{\prime}=m_{1} \frac{V_{0}^{\sharp}}{V_{1}}, \quad \bar{l}_{1}^{\prime}=\bar{m}_{1} \frac{V_{0}}{V_{1}}, \quad l_{i}^{\prime}=m_{i} \frac{V_{i-1} W_{i}}{V_{i} W_{i-1}}, \quad \bar{l}_{i}^{\prime}=\bar{m}_{i} \frac{V_{i-1}}{V_{i}} \quad(2 \leq i \leq n), \\
& m_{1}^{\prime}=l_{1} \frac{V_{0}}{V_{1}^{*}}, \quad \bar{m}_{1}^{\prime}=\bar{l}_{1} \frac{V_{0}^{\sharp}}{V_{1}^{*}}, \quad m_{i}^{\prime}=l_{i} \frac{V_{i-1}^{*}}{V_{i}^{*}}, \quad \bar{m}_{i}^{\prime}=\bar{l}_{i} \frac{V_{i-1}^{*} W_{i}}{V_{i}^{*} W_{i-1}} \quad(2 \leq i \leq n) .
\end{aligned}
$$

Here note that for $\left(l^{\prime}, m^{\prime}\right)=\mathcal{R}(l, m)$ we have $l_{1}^{\prime} l_{2}^{\prime} \cdots \bar{l}_{2}^{\prime} \bar{l}_{1}^{\prime}=M$ and $m_{1}^{\prime} m_{2}^{\prime} \cdots \bar{m}_{2}^{\prime} \bar{m}_{1}^{\prime}=$ $L$.

We shall describe the tropical $\mathrm{R}$ map on $\mathcal{V}\left(B_{n}^{(1)}\right)_{L} \times \mathcal{V}\left(B_{n}^{(1)}\right)_{M}$ defined by $\overline{\mathcal{R}}(x, y):=(\Xi, \Xi) \circ \mathcal{R}\left(B_{n}^{(1)}\right) \circ\left(\Xi^{-1}, \Xi^{-1}\right)(x, y)\left(x \in \mathcal{V}\left(B_{n}^{(1)}\right)_{L}, y \in \mathcal{V}\left(B_{n}^{(1)}\right)_{M}\right)$. Restricting the rational functions $\bar{V}_{i}$ and $\bar{W}_{i}$ for $D_{n+1}^{(1)}$ to $x_{n}=x_{n+1}$ and $y_{n}=y_{n+1}$, we define the rational functions $\bar{V}_{i}(i=0,1, \ldots, n)$ and $\bar{W}_{i}(i=1, \ldots, n)$ on $\mathcal{V}\left(B_{n}^{(1)}\right)_{L} \times \mathcal{V}\left(B_{n}^{(1)}\right)_{M}\left(L, M \in \mathbb{C}^{\times}\right)$by

$$
\bar{W}_{i}:=\bar{V}_{i} \bar{V}_{i}^{*}+(M-L) \bar{V}_{i}^{*}+(L-M) \bar{V}_{i} \quad(1 \leq i \leq n-1), \quad \bar{W}_{n}:=\bar{V}_{n} \bar{V}_{n}^{*} .
$$

$$
\bar{V}_{i}=\sum_{j=1}^{n-1}\left(\bar{\theta}_{i, j}(x, y)+\bar{\theta}_{i, j}^{\prime}(x, y)\right)+\sum_{j=1}^{n+1}\left(\bar{\eta}_{i, j}(x, y)+\bar{\eta}_{i, j}^{\prime}(x, y)\right) \text {, }
$$


where $\sharp$ is the involution on $\mathcal{V}\left(B_{n}^{(1)}\right)_{L}$ and $*$ is the involution $\mathcal{V}\left(B_{n}^{(1)}\right)_{L} \times \mathcal{V}\left(B_{n}^{(1)}\right)_{M} \rightarrow$ $\mathcal{V}\left(B_{n}^{(1)}\right)_{M} \times \mathcal{V}\left(B_{n}^{(1)}\right)_{L}$ defined by

$$
\begin{aligned}
\sharp \quad: \quad x_{i} & \mapsto \frac{x_{i}}{L x_{1} \bar{x}_{1}} \quad(i=1, \cdots, n), \quad \bar{x}_{i} \mapsto \frac{\bar{x}_{i}}{L x_{1} \bar{x}_{1}} \quad(i=1, \cdots, n-1), \\
* \quad: \quad x_{i} & \mapsto \frac{1}{M \bar{y}_{i}}, \quad \bar{x}_{i} \mapsto \frac{1}{M y_{i}} \quad(i=1, \cdots, n-1), \quad x_{n} \mapsto \frac{1}{M y_{n}}, \\
y_{i} & \mapsto \frac{1}{L \bar{x}_{i}}, \quad \bar{y}_{i} \mapsto \frac{1}{L x_{i}} \quad(i=1, \cdots, n-1), \quad y_{n} \mapsto \frac{1}{L x_{n}},
\end{aligned}
$$

and

$$
\begin{aligned}
& \bar{\theta}_{i, j}(x, y):=L \frac{x_{i} y_{j}}{x_{j} y_{i}} \quad(j \leq i \leq n, j<n), \quad \bar{\theta}_{i, j}(x, y):=M \frac{x_{i} y_{j}}{x_{j} y_{i}} \quad(i<j<n), \\
& \bar{\theta}_{i, j}^{\prime}(x, y):=M \frac{x_{i} \bar{y}_{j}}{\bar{x}_{j} y_{i}} \quad(1 \leq j<n), \\
& \bar{\eta}_{i, j}(x, y):=L^{1-\delta_{j, 1}} \frac{x_{i} \bar{x}_{j-1} y_{j-1}}{x_{j} \bar{x}_{j} y_{i}} \quad(1 \leq j \leq i \leq n, j<n), \\
& \bar{\eta}_{i, j}(x, y):=\frac{M}{L^{\delta_{j, 1}}} \frac{x_{i} \bar{x}_{j-1} y_{j-1}}{x_{j} \bar{x}_{j} y_{i}} \quad(0 \leq i<j<n), \\
& \bar{\eta}_{i, n}(x, y)=M \frac{x_{i} \bar{x}_{n-1} y_{n-1}}{x_{n}^{2} y_{i}} \quad(0 \leq i \leq n), \\
& \bar{\eta}_{i, n+1}(x, y):=M \frac{x_{i} y_{n}}{x_{n} y_{i}} \quad(i \leq n), \\
& \bar{\eta}_{i, j}^{\prime}(x, y):=L^{\delta_{j, 1}} M \frac{x_{i} y_{j} \bar{y}_{j}}{\bar{x}_{j-1} y_{j-1} y_{i}} \quad(1 \leq j<n, i \leq n), \quad \bar{\eta}_{n, n+1}^{\prime}(x, y):=L, \\
& \bar{\eta}_{i, n}^{\prime}(x, y):=M \frac{x_{i} y_{n}^{2}}{\bar{x}_{n-1} y_{n-1} y_{i}} \quad(0 \leq i \leq n), \\
& \bar{\eta}_{i, n+1}^{\prime}(x, y):=M \frac{x_{i} y_{n}}{y_{i} x_{n}} \quad(0 \leq i<n),
\end{aligned}
$$

where we understand $x_{0}=\bar{x}_{0}=y_{0}=\bar{y}_{0}=1$. Note that as above e.g.,

$$
\bar{\theta}_{i, j}(x, y)^{*}:=\bar{\theta}_{i, j}^{L M}(x, y)^{*}=\bar{\theta}_{i, j}^{M L}\left((x, y)^{*}\right) .
$$

Here we define $\overline{\mathcal{R}}(x, y)=\overline{\mathcal{R}}\left(B_{n}^{(1)}\right)(x, y)=\left(x^{\prime}, y^{\prime}\right)$ where

$$
\begin{aligned}
& x_{i}^{\prime}:=y_{i} \frac{\bar{V}_{i}}{\bar{V}_{0}}(1 \leq i \leq n-1) \quad x_{n}^{\prime}:=y_{n} \frac{\bar{V}_{n}}{\bar{V}_{0}}, \quad \bar{x}_{1}^{\prime}:=\bar{y}_{1} \frac{\bar{V}_{0}^{\sharp}}{\bar{V}_{1}}, \quad \bar{x}_{i}^{\prime}:=\bar{y}_{i} \frac{\bar{V}_{0}^{\sharp} \bar{W}_{i}}{\bar{V}_{i} \bar{W}_{1}}(i \geq 2), \\
& y_{1}^{\prime}:=x_{1} \frac{\bar{V}_{1}^{*}}{\bar{V}_{0}^{\sharp}}, y_{i}^{\prime}:=x_{i} \frac{\bar{V}_{i}^{*} \bar{W}_{1}}{\bar{V}_{0}^{\sharp} \bar{W}_{i}}(i>1), \quad \bar{y}_{i}^{\prime}:=\bar{x}_{i} \frac{\bar{V}_{0}}{\bar{V}_{i}^{*}}(1 \leq i \leq n-1), \quad y_{n}^{\prime}:=x_{n} \frac{\bar{V}_{n} \bar{W}_{1}}{\bar{V}_{0}^{\sharp} \bar{W}_{n}} .
\end{aligned}
$$

9.4. Tropical $\mathbf{R}$ for $D_{n+1}^{(2)}(n \geq 2)$. As in the previous subsection, we shall describe tropical R maps of type $D_{n+1}^{(2)}$. We see the following lemma for $\left\{X_{3}^{(n, L)}\right\}_{L \in \mathbb{C}^{\times}}$.

Lemma 9.4. The birational map $R\left(D_{n+1}^{(2)}\right)$ is a tropical $R$ map on $\left\{X_{3}^{(n, L)}\right\}_{L}$.

The proof is the same as the one for Lemma 9.3 .

Let us describe the explicit form of tropical $\mathrm{R}$ on $\mathcal{B}_{L}\left(D_{n+1}^{(2)}\right)$ as in the previous subsection. Set $\mathcal{R}\left(D_{n+1}^{(2)}\right):=\left(\eta^{-1}, \eta^{-1}\right) \circ R\left(D_{n+1}^{(2)}\right) \circ(\eta, \eta)$ where $\eta$ is as in 6.4 Let 
$*: \mathcal{B}_{L}\left(D_{n+1}^{(2)}\right) \times \mathcal{B}_{M}\left(D_{n+1}^{(2)}\right) \rightarrow \mathcal{B}_{M}\left(D_{n+1}^{(2)}\right) \times \mathcal{B}_{L}\left(D_{n+1}^{(2)}\right)$ be an involution defined by

$$
\begin{aligned}
& \left(\left(l_{0}, l_{1}, \ldots, \bar{l}_{2}, \bar{l}_{1}\right),\left(m_{0}, m_{1}, \ldots, \bar{m}_{2}, \bar{m}_{1}\right)\right)^{*} \\
& \quad=\left(\left(m_{0}, \bar{m}_{1}, \bar{m}_{2}, \ldots, m_{2}, m_{1}\right),\left(l_{0}, \bar{l}_{1}, \bar{l}_{2}, \ldots, l_{2}, l_{1}\right)\right),
\end{aligned}
$$

that is, $*: l_{0} \leftrightarrow m_{0}$ and $l_{i} \leftrightarrow \bar{m}_{i}, \quad \bar{l}_{i} \leftrightarrow m_{i}(1 \leq i \leq n)$.

Restricting the functions $V_{i}$ and $W_{i}$ for $B_{n+1}^{(1)}$ to $l_{1}=\bar{l}_{1}$ and $m_{1}=\bar{m}_{1}$, and replacing $X_{i}$ with $X_{i-1}$ where $X=l, \bar{l}, m$, and $\bar{m}$, we define the rational functions $V_{i}$ $(i=0,1, \ldots, n+1)$ and $W_{i}(i=1, \ldots, n+1)$ on $\mathcal{B}_{L}\left(D_{n+1}^{(2)}\right) \times \mathcal{B}_{M}\left(D_{n+1}^{(2)}\right)\left(L, M \in \mathbb{C}^{\times}\right)$ as

$$
\begin{gathered}
W_{i}:=V_{i} V_{i}^{*}+(M-L) V_{i}^{*}+(L-M) V_{i} \quad(1 \leq i \leq n), \quad W_{n+1}:=V_{n+1} V_{n+1}^{*} . \\
V_{i}=\sum_{j=1}^{n}\left(\theta_{i, j}(l, m)+\theta_{i, j}^{\prime}(l, m)\right)+\sum_{j=1}^{n+2}\left(\eta_{i, j}(l, m)+\eta_{i, j}^{\prime}(l, m)\right)
\end{gathered}
$$

where $L=l_{0}^{2} l_{1} l_{2} \cdots \bar{l}_{2} \bar{l}_{1}, M=m_{0}^{2} m_{1} m_{2} \cdots \bar{m}_{2} \bar{m}_{1}$,

$$
\theta_{i, j}(l, m)= \begin{cases}L \prod_{k=j}^{i-1} \frac{\bar{m}_{k}}{\bar{l}_{k}} & \text { for } 1 \leq j \leq i \leq n+1, \\ M \prod_{k=i}^{j-1} \frac{\bar{l}_{k}}{\bar{m}_{k}} & \text { for } 0 \leq i<j \leq n\end{cases}
$$

$$
\begin{aligned}
& \theta_{i, j}^{\prime}(l, m)=L\left(\prod_{k=0}^{i-1} \frac{\bar{m}_{k}}{\bar{l}_{k}}\right)\left(\prod_{k=0}^{j-1} \frac{m_{k}}{l_{k}}\right) \quad \text { for } \quad j=1, \ldots, n \\
& \eta_{i, j}(l, m)= \begin{cases}L\left(\prod_{k=j}^{i-1} \frac{\bar{m}_{k}}{\bar{l}_{k}}\right)\left(\frac{\bar{m}_{j-1}}{l_{j-1}}\right) & \text { for } \quad 1 \leq j \leq i \leq n+1, \\
M\left(\prod_{k=i}^{j-1} \frac{\bar{l}_{k}}{\bar{m}_{k}}\right)\left(\frac{\bar{m}_{j-1}}{l_{j-1}}\right) & \text { for } \quad i+1 \leq j \leq n+1, \\
M\left(\prod_{k=i}^{n} \frac{\bar{l}_{k}}{\bar{m}_{k}}\right) & \text { for } \quad j=n+2,\end{cases} \\
& \eta_{i, j}^{\prime}(l, m)= \begin{cases}L\left(\prod_{k=0}^{i-1} \frac{\bar{m}_{k}}{\bar{l}_{k}}\right)\left(\prod_{k=0}^{j-1} \frac{m_{k}}{l_{k}}\right)\left(\frac{l_{j-1}}{\bar{m}_{j-1}}\right) \quad \text { for } \quad 1 \leq j \leq n+1, \\
L\left(\frac{L}{M}\right)^{\delta_{i, n+1}}\left(\prod_{k=0}^{i-1} \frac{\bar{m}_{k}}{\bar{l}_{k}}\right)\left(\prod_{k=0}^{n} \frac{m_{k}}{l_{k}}\right) \quad \text { for } \quad j=n+2,\end{cases}
\end{aligned}
$$

where we understand $\bar{l}_{0}=l_{0}, \bar{m}_{0}=m_{0}$. Now, we define the tropical $\mathrm{R}$ map $\mathcal{R}\left(D_{n+1}^{(2)}\right)$ on $\mathcal{B}_{L}\left(D_{n+1}^{(2)}\right) \times \mathcal{B}_{M}\left(D_{n+1}^{(2)}\right)$ by

$$
\begin{aligned}
& \mathcal{R}\left(D_{n+1}^{(2)}\right)(l, m)=\left(l^{\prime}, m^{\prime}\right) \quad \text { where } \\
& l_{0}^{\prime}=m_{0} \frac{V_{0}}{V_{1}}, \quad l_{i}^{\prime}=m_{i} \frac{V_{i} W_{i+1}}{V_{i+1} W_{i}}, \quad \bar{l}_{i}^{\prime}=\bar{m}_{i} \frac{V_{i}}{V_{i+1}} \quad(1 \leq i \leq n), \\
& m_{0}^{\prime}=l_{0} \frac{V_{0}}{V_{1}^{*}}, \quad m_{i}^{\prime}=l_{i} \frac{V_{i}^{*}}{V_{i+1}^{*}}, \quad \bar{m}_{i}^{\prime}=\bar{l}_{i} \frac{V_{i}^{*} W_{i+1}}{V_{i+1}^{*} W_{i}} \quad(1 \leq i \leq n) .
\end{aligned}
$$


Here note that for $\left(l^{\prime}, m^{\prime}\right)=\mathcal{R}\left(D_{n+1}^{(2)}\right)(l, m)$ we have $l_{0}^{\prime 2} l_{1}^{\prime} l_{2}^{\prime} \cdots \bar{l}_{2}^{\prime} \bar{l}_{1}^{\prime}=M$ and $m_{0}^{\prime 2} m_{1}^{\prime} m_{2}^{\prime} \cdots \bar{m}_{2}^{\prime} \bar{m}_{1}^{\prime}=L$.

Next, we shall describe the tropical $\mathrm{R}$ on $\mathcal{V}\left(D_{n+1}^{(2)}\right)_{L} \times \mathcal{V}\left(D_{n+1}^{(2)}\right)_{M}$ defined by $\overline{\mathcal{R}}(x, y):=(\Xi, \Xi) \circ \mathcal{R}\left(D_{n+1}^{(2)}\right) \circ\left(\Xi^{-1}, \Xi^{-1}\right)(x, y)\left(x \in \mathcal{V}\left(D_{n+1}^{(2)}\right)_{L}, y \in \mathcal{V}\left(D_{n+1}^{(2)}\right)_{M}\right)$.

In this case, we replace $L$ (resp. $M)$ for $\mathcal{B}_{L}\left(D_{n+1}^{(2)}\right)$ (resp. $\left.\mathcal{B}_{M}\left(D_{n+1}^{(2)}\right)\right)$ with $L^{2}$ (resp. $\left.M^{2}\right)$ since $\mathcal{V}\left(D_{n+1}^{(2)}\right)_{L} \cong \mathcal{B}_{L^{2}}\left(D_{n+1}^{(2)}\right)$ as in 6.4.

We define the rational functions $\bar{V}_{i}(x, y)(i=0,1, \ldots, n+1)$ and $\bar{W}_{i}(x, y)(i=$ $1, \ldots, n+1)$ on $\mathcal{V}\left(D_{n+1}^{(2)}\right)_{L} \times \mathcal{V}\left(D_{n+1}^{(2)}\right)_{M}\left(L, M \in \mathbb{C}^{\times}\right)$by

$\bar{W}_{i}:=\bar{V}_{i} \bar{V}_{i}^{*}+\left(M^{2}-L^{2}\right) \bar{V}_{i}^{*}+\left(L^{2}-M^{2}\right) \bar{V}_{i} \quad(1 \leq i \leq n), \quad \bar{W}_{n+1}:=\bar{V}_{n+1} \bar{V}_{n+1}^{*}$.

$$
\bar{V}_{i}=\sum_{j=1}^{n}\left(\bar{\theta}_{i, j}(x, y)+\bar{\theta}_{i, j}^{\prime}(x, y)\right)+\sum_{j=1}^{n+2}\left(\bar{\eta}_{i, j}(x, y)+\bar{\eta}_{i, j}^{\prime}(x, y)\right) \text {, }
$$

where $*$ is the involution $\mathcal{V}\left(D_{n+1}^{(2)}\right)_{L} \times \mathcal{V}\left(D_{n+1}^{(2)}\right)_{M} \rightarrow \mathcal{V}\left(D_{n+1}^{(2)}\right)_{M} \times \mathcal{V}\left(D_{n+1}^{(2)}\right)_{L}$ defined by

$$
\begin{aligned}
*: x_{0} & \mapsto y_{0}, x_{i} \mapsto \frac{y_{0}^{2}}{M^{2} \bar{y}_{i}}, \bar{x}_{i} \mapsto \frac{y_{0}^{2}}{M^{2} y_{i}}(i=1, \cdots, n-1), x_{n} \mapsto \frac{y_{0}^{2}}{M^{2} y_{n}}, \\
y_{0} & \mapsto x_{0}, y_{i} \mapsto \frac{x_{0}^{2}}{L^{2} \bar{x}_{i}}, \bar{y}_{i} \mapsto \frac{x_{0}^{2}}{L^{2} x_{i}}(i=1, \cdots, n-1), y_{n} \mapsto \frac{x_{0}^{2}}{L^{2} x_{n}} .
\end{aligned}
$$

and

$$
\begin{aligned}
& \bar{\theta}_{i, j}(x, y):= \begin{cases}L^{2}\left(\frac{x_{i-1}}{y_{i-1}}\right)^{1+\delta_{i, 1}}\left(\frac{y_{j-1}}{x_{j-1}}\right)^{1+\delta_{j, 1}} & (1 \leq j \leq i \leq n+1, j \leq n), \\
M^{2}\left(\frac{x_{0}}{y_{0}}\right)^{\delta_{i, 0}+\delta_{i, 1}-\delta_{j, 1}} \frac{x_{i-1} y_{j-1}}{x_{j-1} y_{i-1}} & (0 \leq i<j \leq n),\end{cases} \\
& \bar{\theta}_{i, j}^{\prime}(x, y):=M^{2}\left(\frac{L^{2}}{M^{2}}\right)^{\delta_{j, 1}}\left(\frac{x_{0}}{y_{0}}\right)^{\delta_{i, 0}+\delta_{i, 1}} \frac{x_{i-1} \bar{y}_{j-1}}{\bar{x}_{j-1} y_{i-1}} \quad(1 \leq j \leq n), \\
& \bar{\eta}_{i, j}(x, y):= \begin{cases}L^{2\left(1-\delta_{j, 2}\right)} y_{0}^{\delta_{j, 1}+\delta_{j, 2}}\left(\frac{x_{0}}{y_{0}}\right)^{\delta_{i, 1} \delta_{j, 1}} \frac{x_{i-1} \bar{x}_{j-2} y_{j-2}}{x_{j-1} \bar{x}_{j-1} y_{i-1}} & (1 \leq j \leq i \leq n+1), \\
\frac{M^{2}}{L^{2 \delta_{j, 2}}}\left(\frac{x_{0}}{y_{0}^{1-\delta_{j, 1}-\delta_{j, 2}}}\right)^{\delta_{i, 0}+\delta_{i, 1}} \frac{x_{i-1} \bar{x}_{j-2} y_{j-2}}{x_{j-1} \bar{x}_{j-1} y_{i-1}} & (i+1 \leq j \leq n+1), \\
M^{2}\left(\frac{x_{0}}{y_{0}}\right)^{\delta_{i, 0}+\delta_{i, 1}} \frac{x_{i-1} y_{n}}{x_{n} y_{i-1}} & (j=n+2),\end{cases} \\
& \bar{\eta}_{i, j}^{\prime}(x, y):= \begin{cases}\bar{\theta}_{i, j}^{\prime}(x, y) \times\left(\frac{L^{2}}{y_{0}}\right)^{\delta_{j, 2}} \frac{\bar{x}_{j-1} y_{j-1}}{\bar{x}_{j-2} y_{j-2}} & (1 \leq j \leq n), \\
M^{2}\left(\frac{x_{0}}{y_{0}}\right)^{\delta_{i, 0}+\delta_{i, 1}} \frac{x_{i-1} y_{n}^{2}}{y_{i-1} \bar{x}_{n-1} y_{n-1}} & (j=n+1), \\
M^{2}\left(\frac{L^{2}}{M^{2}}\right)^{\delta_{i, n+1}}\left(\frac{x_{0}}{y_{0}}\right)^{\delta_{i, 0}+\delta_{i, 1}} \frac{x_{i-1} y_{n}}{y_{i-1} x_{n}} & (j=n+2),\end{cases}
\end{aligned}
$$

where we understand $x_{-1}=\bar{x}_{0}=y_{-1}=\bar{y}_{0}=1$ and $\bar{x}_{n}=x_{n}, \bar{y}_{n}=y_{n}$. 
Here we define $\overline{\mathcal{R}}(x, y)=\overline{\mathcal{R}}\left(D_{n+1}^{(2)}\right)(x, y)=\left(x^{\prime}, y^{\prime}\right)$ by

$$
\begin{aligned}
& x_{0}^{\prime}:=y_{0} \frac{\bar{V}_{1}}{\bar{V}_{0}} x_{i}^{\prime}:=y_{i} \frac{\bar{V}_{1} \bar{V}_{i+1}}{\bar{V}_{0}^{2}}(1 \leq i \leq n), \\
& \bar{x}_{i}^{\prime}:=\bar{y}_{i} \frac{\bar{V}_{1} \bar{W}_{i+1}}{\bar{V}_{i+1} \bar{W}_{1}} \quad(1 \leq i \leq n-1), \\
& y_{0}^{\prime}:=x_{0} \frac{\bar{V}_{1}^{*}}{\bar{V}_{0}}, y_{i}^{\prime}:=x_{i} \frac{\bar{V}_{1}^{*} \bar{V}_{i+1}^{*} \bar{W}_{1}}{\bar{V}_{0}^{2} \bar{W}_{i+1}}(1 \leq i \leq n), \\
& \bar{y}_{i}^{\prime}:=\bar{x}_{i} \frac{\bar{V}_{1}^{*}}{\bar{V}_{i+1}^{*}}(1 \leq i \leq n-1) .
\end{aligned}
$$

9.5. Tropical $\mathbf{R}$ for $A_{2 n-1}^{(2)}(n \geq 3)$. We shall describe tropical R's of type $A_{2 n-1}^{(2)}$. We see the following lemma for $\left\{X_{2}^{(n, L)}\right\}_{L \in \mathbb{C}^{\times}}$.

Lemma 9.5. The birational map $R\left(A_{2 n-1}^{(2)}\right)$ is a tropical $R$ map on $\left\{X_{2}^{(n, L)}\right\}_{L}$.

The proof is the same as the one for Lemma 9.3 .

Let us describe the explicit form of tropical $\mathrm{R}$ on $\mathcal{B}_{L}\left(A_{2 n-1}^{(2)}\right)$. Set $\mathcal{R}\left(A_{2 n-1}^{(2)}\right):=$ $\left(\eta^{-1}, \eta^{-1}\right) \circ R\left(A_{2 n-1}^{(2)}\right) \circ(\eta, \eta)$ where $\eta$ is as in 6.5

Let $\sharp$ be the involution on $\mathcal{B}_{L}\left(A_{2 n-1}^{(2)}\right)$ defined by $\sharp: l_{1} \leftrightarrow \bar{l}_{1}$ for $l=\left(l_{1}, \cdots, \bar{l}_{1}\right) \in$ $\mathcal{B}_{L}\left(A_{2 n-1}^{(2)}\right)$ and $*: \mathcal{B}_{L}\left(A_{2 n-1}^{(2)}\right) \times \mathcal{B}_{M}\left(A_{2 n-1}^{(2)}\right) \rightarrow \mathcal{B}_{M}\left(A_{2 n-1}^{(2)}\right) \times \mathcal{B}_{L}\left(A_{2 n-1}^{(2)}\right)$ an involution defined by

$$
\begin{aligned}
\left(\left(l_{1}, l_{2}, \ldots, \bar{l}_{2}, \bar{l}_{1}\right),\left(m_{1}, m_{2}, \ldots, \bar{m}_{2}, \bar{m}_{1}\right)\right)^{*} & \\
= & \left(\left(\bar{m}_{1}, \bar{m}_{2}, \ldots, m_{2}, m_{1}\right),\left(\bar{l}_{1}, \bar{l}_{2}, \ldots, l_{2}, l_{1}\right)\right)
\end{aligned}
$$

that is, $*: l_{i} \leftrightarrow \bar{m}_{i}, \quad \bar{l}_{i} \leftrightarrow m_{i}(1 \leq i \leq n)$.

Restricting the functions $V_{i}$ and $W_{i}$ for $D_{2 n}^{(1)}$ to $X_{2}^{\left(n, L^{2}\right)} \times X_{2}^{\left(n, M^{2}\right)}$, we define the rational functions $V_{i}(i=0,1, \ldots, n)$ and $W_{i}(i=1, \ldots, n)$ on $\mathcal{B}_{L}\left(A_{2 n-1}^{(2)}\right) \times$ $\mathcal{B}_{M}\left(A_{2 n-1}^{(2)}\right)\left(L, M \in \mathbb{C}^{\times}\right)$as

$$
\begin{aligned}
W_{i} & :=V_{i} V_{i}^{*}+\left(M^{2}-L^{2}\right) V_{i}^{*}+\left(L^{2}-M^{2}\right) V_{i} \quad(1 \leq i \leq n), \\
V_{i} & =\sum_{j=1}^{2 n-2}\left(\theta_{i, j}(l, m)+\theta_{i, j}^{\prime}(l, m)\right)+\sum_{j=1}^{2 n}\left(\eta_{i, j}(l, m)+\eta_{i, j}^{\prime}(l, m)\right),
\end{aligned}
$$

where $L^{2}=l_{n} \bar{l}_{n}\left(\prod_{i=1}^{n-1} l_{i} \bar{l}_{i}\right)^{2}, M^{2}=m_{n} \bar{m}_{n}\left(\prod_{i=1}^{n-1} m_{i} \bar{m}_{i}\right)^{2}, \Delta:=\frac{1+\bar{\mu}(l)}{1+\bar{\mu}(m)}$, and 


$$
\begin{aligned}
& \theta_{i, j}(l, m)=\left\{\begin{array}{l}
L^{2} \prod_{k=j+1}^{i} \frac{\bar{m}_{k}}{\bar{l}_{k}} \Delta^{\delta_{i, n}-\delta_{j, n}} \quad 1 \leq j \leq i \leq n, \\
M^{2} \prod_{k=i+1}^{j} \frac{\bar{l}_{k}}{\bar{m}_{k}} \Delta^{-\delta_{j, n}} \quad 0 \leq i<j \leq n, \\
L M\left(\prod_{k=1}^{i} \frac{\bar{m}_{k}}{\bar{l}_{k}}\right)\left(\prod_{k=1}^{2 n-j-1} \frac{m_{k}}{l_{k}}\right) \Delta^{\delta_{i, n}} \frac{\bar{l}_{2 n-j}\left(m_{2 n-j}+\bar{m}_{2 n-j}\right)}{\left(l_{2 n-j}+\bar{l}_{2 n-j}\right) \bar{m}_{2 n-j}} \\
0 \leq i \leq n<j \leq 2 n-2 .
\end{array}\right. \\
& \theta_{i, j}^{\prime}(l, m)=\left\{\begin{array}{l}
L^{2}\left(\prod_{k=1}^{i} \frac{\bar{m}_{k}}{\bar{l}_{k}}\right)\left(\prod_{k=1}^{j} \frac{m_{k}}{l_{k}}\right) \Delta^{\delta_{i, n}}\left(\frac{1+\mu(l)}{1+\mu(m)}\right)^{\delta_{j, n}} \quad j \leq n, \\
L M\left(\prod_{k=1}^{i} \frac{\bar{m}_{k}}{\bar{l}_{k}}\right)\left(\prod_{k=1}^{2 n-j-1} \frac{\bar{l}_{k}}{\bar{m}_{k}}\right) \Delta^{\delta_{i, n}} \frac{m_{2 n-j}\left(l_{2 n-j}+\bar{l}_{2 n-j}\right)}{l_{2 n-j}\left(m_{2 n-j}+\bar{m}_{2 n-j}\right)} \quad n<j .
\end{array}\right. \\
& \left\{\begin{array}{l}
L^{2}\left(\prod_{k=j+1}^{i} \frac{\bar{m}_{k}}{\bar{l}_{k}}\right)\left(\frac{\bar{m}_{j}}{l_{j}}\right) \Delta^{\delta_{i, n}} \mu(l)^{\delta_{j, n}} \quad 1 \leq j \leq i \leq n, \\
M^{2}\left(\prod_{k=i+1}^{j} \frac{\bar{l}_{k}}{\bar{m}_{k}}\right)\left(\frac{\bar{m}_{j}}{l_{j}}\right) \mu(l)^{\delta_{j, n}} \quad i<j \leq n,
\end{array}\right. \\
& \eta_{i, j}(l, m)=\left\{L M\left(\prod_{k=1}^{i} \frac{\bar{m}_{k}}{\bar{l}_{k}}\right)\left(\prod_{k=1}^{n-1} \frac{m_{k}}{l_{k}}\right) \Delta^{\delta_{i, n}} \frac{1+\mu(m)}{1+\bar{\mu}(l)} \quad i \leq n, j=n+1\right. \\
& \operatorname{LM}\left(\prod_{k=1}^{i} \frac{\bar{m}_{k}}{\bar{l}_{k}}\right)\left(\prod_{k=1}^{2 n-j} \frac{m_{k}}{l_{k}}\right) \Delta^{\delta_{i, n}} \frac{\left(m_{2 n-j+1}+\bar{m}_{2 n-j+1}\right) l_{2 n-j+1}}{\left(l_{2 n-j+1}+\bar{l}_{2 n-j+1}\right) \bar{m}_{2 n-j+1}} \\
& L M\left(\prod_{k=1}^{i} \frac{\bar{m}_{k}}{\bar{l}_{k}}\right) \frac{\bar{l}_{1}}{l_{1}} \Delta^{\delta_{i, n}} \quad i<j=2 n . \\
& i<n+1<j \leq 2 n-1 \text {, } \\
& L^{2}\left(\prod_{k=1}^{i} \frac{\bar{m}_{k}}{\bar{l}_{k}}\right)\left(\prod_{k=1}^{j} \frac{m_{k}}{l_{k}}\right)\left(\frac{l_{j}}{\bar{m}_{j}}\right) \Delta^{\delta_{i, n}} \bar{\mu}(m)^{\delta j, n} \quad 1 \leq j \leq n, \\
& L^{2}\left(\prod_{k=1}^{i} \frac{\bar{m}_{k}}{\bar{l}_{k}}\right)\left(\prod_{k=1}^{n} \frac{m_{k}}{l_{k}}\right) \Delta^{\delta_{i, n}} \frac{1+\mu(l)}{(1+\mu(m)) \mu(m)} \quad j=n+1, \\
& \eta_{i, j}^{\prime}(l, m)=\left\{\begin{array}{l}
L M\left(\prod_{k=1}^{i} \frac{\bar{m}_{k}}{\bar{l}_{k}}\right)\left(\prod_{k=1}^{2 n-j} \frac{\bar{l}_{k}}{\bar{m}_{k}}\right) \Delta^{\delta_{i, n}} \frac{\left(l_{2 n-j+1}+\bar{l}_{2 n-j+1}\right) \bar{m}_{2 n-j+1}}{\left(m_{2 n-j+1}+\bar{m}_{2 n-j+1}\right) l_{2 n-j+1}}
\end{array}\right. \\
& L M\left(\prod_{k=1}^{i} \frac{\bar{m}_{k}}{\bar{l}_{k}}\right) \Delta^{\delta_{i, n}} \frac{m_{1}}{\bar{m}_{1}} \quad j=2 n .
\end{aligned}
$$


Now, we define the tropical $\mathrm{R}$

$$
\mathcal{R}\left(A_{2 n-1}^{(2)}\right): \mathcal{B}_{L}\left(A_{2 n-1}^{(2)}\right) \times \mathcal{B}_{M}\left(A_{2 n-1}^{(2)}\right) \rightarrow \mathcal{B}_{M}\left(A_{2 n-1}^{(2)}\right) \times \mathcal{B}_{L}\left(A_{2 n-1}^{(2)}\right)
$$

by

$$
\mathcal{R}\left(A_{2 n-1}^{(2)}\right)(l, m)=\left(l^{\prime}, m^{\prime}\right)
$$

where

$$
\begin{aligned}
& l_{1}^{\prime}=m_{1} \frac{V_{0}^{\sharp}}{V_{1}}, \quad l_{i}^{\prime}=m_{i} \frac{V_{i-1} W_{i}}{V_{i} W_{i-1}}, \quad \bar{l}_{i}^{\prime}=\bar{m}_{i} \frac{V_{i-1}}{V_{i}} \quad(1 \leq i \leq n-1), \\
& l_{n}^{\prime}=\frac{m_{n} V_{n-1} W_{n}}{(1+\mu(m)) V_{n} W_{n-1}}\left(1+\frac{m_{n} W_{n}}{\bar{m}_{n} W_{n-1}} \bar{\mu}(m)\right), \\
& \bar{l}_{n}^{\prime}=\frac{\bar{m}_{n} V_{n-1}}{(1+\bar{\mu}(m)) V_{n}}\left(1+\frac{\bar{m}_{n} W_{n-1}}{m_{n} W_{n}} \mu(m)\right), \\
& m_{1}^{\prime}=l_{1} \frac{V_{0}}{V_{1}^{*}}, \quad \bar{m}_{1}^{\prime}=\bar{l}_{1} \frac{V_{0}^{\sharp}}{V_{1}^{*}}, \quad m_{i}^{\prime}=l_{i} \frac{V_{i-1}^{*}}{V_{i}^{*}}, \quad \bar{m}_{i}^{\prime}=\bar{l}_{i} \frac{V_{i-1}^{*} W_{i}^{(n)}}{V_{i}^{*} W_{i-1}^{(n)}} \quad(2 \leq i \leq n-1), \\
& m_{n}^{\prime}=\frac{l_{n} V_{n-1}^{*}}{(1+\mu(l)) V_{n}^{*}}\left(1+\frac{l_{n} W_{n-1}}{\bar{l}_{n} W_{n}} \bar{\mu}(l)\right), \\
& \bar{m}_{n}^{\prime}=\frac{\bar{l}_{n} V_{n-1}^{*} W_{n}}{(1+\bar{\mu}(l)) V_{n}^{*} W_{n-1}}\left(1+\frac{\bar{l}_{n} W_{n}}{l_{n} W_{n-1}} \mu(l)\right) .
\end{aligned}
$$

Here note that for $\left(l^{\prime}, m^{\prime}\right)=\mathcal{R}\left(A_{2 n-1}^{(2)}\right)(l, m)$ we have $\left(l_{1}^{\prime} l_{2}^{\prime} \cdots l_{n-1}^{\prime} \bar{l}_{n-1}^{\prime} \ldots \bar{l}_{2}^{\prime} \bar{l}_{1}^{\prime}\right)^{2} l_{n}^{\prime} \bar{l}_{n}^{\prime}$ $=M^{2}$ and $\left(m_{1}^{\prime} m_{2}^{\prime} \cdots m_{n-1}^{\prime} \bar{m}_{n-1}^{\prime} \cdots \bar{m}_{2}^{\prime} \bar{m}_{1}^{\prime}\right)^{2} m_{n}^{\prime} \bar{m}_{n}^{\prime}=L^{2}$.

Next, we shall describe tropical $\mathrm{R}$ on $\mathcal{V}\left(A_{2 n-1}^{(2)}\right)_{L} \times \mathcal{V}\left(A_{2 n-1}^{(2)}\right)_{M}$. Let $*$ be the involution $\mathcal{V}\left(A_{2 n-1}^{(2)}\right)_{L} \times \mathcal{V}\left(A_{2 n-1}^{(2)}\right)_{M} \rightarrow \mathcal{V}\left(A_{2 n-1}^{(2)}\right)_{M} \times \mathcal{V}\left(A_{2 n-1}^{(2)}\right)_{L}$ defined by

$$
* \quad: \quad x_{i} \mapsto \frac{1}{M^{2} \bar{y}_{i}}, \bar{x}_{i} \mapsto \frac{1}{M^{2} y_{i}}, y_{i} \mapsto \frac{1}{L^{2} \bar{x}_{i}}, \bar{y}_{i} \mapsto \frac{1}{L^{2} x_{i}}(i=1, \cdots, n)
$$

and $\sharp$ the involution on $\mathcal{V}\left(A_{2 n-1}^{(2)}\right)_{L}$ defined by

$$
\sharp: x_{i} \mapsto \frac{x_{i}}{L^{2} x_{1} \bar{x}_{1}}, \quad \bar{x}_{i} \mapsto \frac{\bar{x}_{i}}{L^{2} x_{1} \bar{x}_{1}} \quad(i=1, \cdots, n-1), \quad x_{n} \mapsto \frac{x_{n}}{\left(L^{2} x_{1} \bar{x}_{1}\right)^{2}} .
$$


We define the rational functions $\bar{V}_{i}(x, y)(i=0,1, \ldots, n)$ and $\bar{W}_{i}(x, y)(i=1, \ldots, n)$ on $\mathcal{V}\left(A_{2 n-1}^{(2)}\right)_{L} \times \mathcal{V}\left(A_{2 n-1}^{(2)}\right)_{M}\left(L, M \in \mathbb{C}^{\times}\right)$by

$$
\begin{aligned}
& \bar{V}_{i}=\sum_{j=1}^{2 n-2}\left(\bar{\theta}_{i, j}(x, y)+\bar{\theta}_{i, j}^{\prime}(x, y)\right)+\sum_{j=1}^{2 n}\left(\bar{\eta}_{i, j}(x, y)+\bar{\eta}_{i, j}^{\prime}(x, y)\right), \\
& \bar{W}_{i}:=\bar{V}_{i} \bar{V}_{i}^{*}+\left(M^{2}-L^{2}\right) \bar{V}_{i}^{*}+\left(L^{2}-M^{2}\right) \bar{V}_{i} \quad(1 \leq i \leq n), \\
& \mu(x):=\frac{x_{n}}{L x_{n-1} \bar{x}_{n-1}}=\bar{\mu}(x)^{-1}, \mu(y):=\frac{y_{n}}{M y_{n-1} \bar{y}_{n-1}}=\bar{\mu}(y)^{-1}, \\
& \Delta:=\frac{1+\bar{\mu}(x)}{1+\bar{\mu}(y)}, \quad \nabla:=\frac{1+\mu(x)}{1+\mu(y)}, \quad \square:=\frac{1+\mu(y)}{1+\bar{\mu}(x)},
\end{aligned}
$$

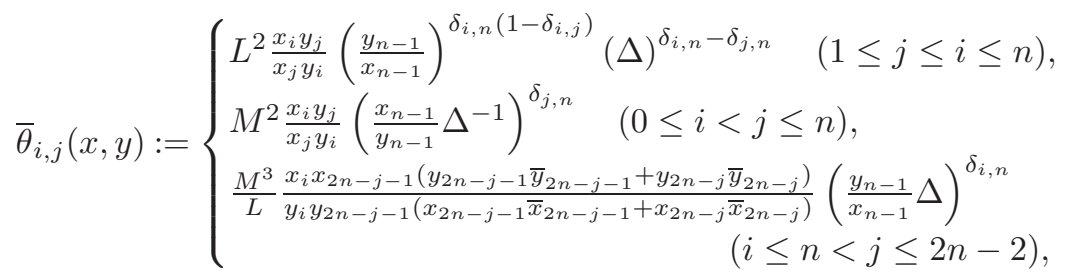

$$
\begin{aligned}
& \bar{\theta}_{i, j}^{\prime}(x, y):=\left\{\begin{array}{l}
M^{2} \frac{x_{i} \bar{y}_{j}}{y_{i} \bar{x}_{j}}\left(\frac{y_{n-1}}{x_{n-1}} \Delta\right)^{\delta_{i, n}}\left(\frac{L^{2} \bar{x}_{n-1}}{M^{2} \bar{y}_{n-1}} \nabla\right)^{\delta_{j, n}} \quad(1 \leq j \leq n), \\
L M \frac{x_{i} y_{2 n-j-1}\left(1+\frac{x_{2 n-j-1} \bar{x}_{2 n-j-1}}{x_{2 n-j} \bar{x}_{2 n-j}}\right)}{y_{i} x_{2 n-j-1}\left(1+\frac{y_{2 n-j-1} \bar{y}_{2 n-j-1}}{y_{2 n-j} \bar{y}_{2 n-j}}\right)}\left(\frac{y_{n-1}}{x_{n-1}} \Delta\right)^{\delta_{i, n}} \quad(n<j \leq 2 n-2),
\end{array}\right.
\end{aligned}
$$

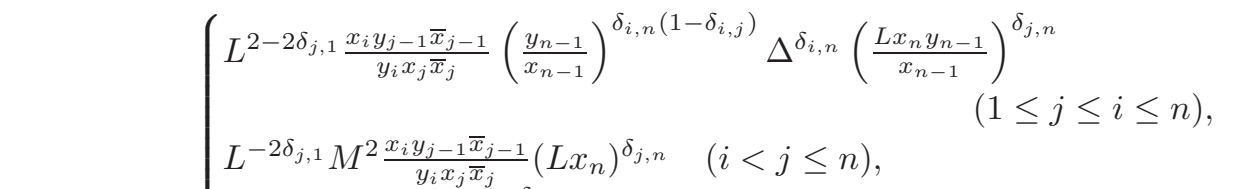

$$
\begin{aligned}
& \bar{\eta}_{i, j}(x, y):=\left\{\begin{array}{l}
\frac{M^{3}}{L} \frac{x_{i} \bar{y}_{n-1}}{y_{i} \bar{x}_{n-1}}\left(\frac{y_{n-1}}{x_{n-1}} \Delta\right)^{\delta_{i, n}} \square \quad(0 \leq i \leq n, j=n+1), \\
\frac{M^{3}}{L} \frac{x_{i} \bar{y}_{2 n-j}}{y_{i} \bar{x}_{2 n-j}} \frac{1+\frac{y_{2 n-j+1} \bar{y}_{2 n-j+1}}{1+\frac{x_{2 n-j} \bar{y}_{2 n-j}}{x_{2 n-j+1} \bar{x}_{2 n-j+1}}}\left(\frac{y_{n-1}}{x_{n-1}} \Delta\right)^{\delta_{i, n}}}{}
\end{array}\right. \\
& \begin{array}{ll} 
& (0 \leq i \leq n<j \leq 2 n-1), \\
\frac{M}{L} \frac{x_{i}}{y_{i} x_{1} \bar{x}_{1}}\left(\frac{y_{n-1}}{x_{n-1}} \Delta\right)^{\delta_{i, n}} \quad(0 \leq i \leq n<j=2 n),
\end{array} \\
& \bar{\eta}_{i, j}^{\prime}(x, y):= \begin{cases}L^{2 \delta_{j, 1}} M^{2} \frac{x_{i} y_{j} \bar{y}_{j}}{y_{i} y_{j-1} \bar{x}_{j-1}}\left(\frac{y_{n-1}}{x_{n-1}} \Delta\right)^{\delta_{i, n}}\left(\frac{1}{M y_{n}}\right)^{\delta_{j, n}} & (1 \leq j \leq n), \\
L^{2} M \frac{x_{i} y_{n-1} \bar{x}_{n-1}}{y_{i} x_{n}}\left(\frac{y_{n-1}}{x_{n-1}} \Delta\right)^{\delta_{i, n}} \nabla & (j=n+1), \\
L M \frac{x_{i} y_{2 n-j}}{y_{i} x_{2 n-j}} \frac{\left(1+\frac{x_{2 n-j} \bar{x}_{2 n-j}}{x_{2 n-j+1} \bar{x}_{2 n-j+1}}\right)}{\left(1+\frac{y_{2 n-j+1} \bar{y}_{2 n-j+1}}{y_{2 n-j} \bar{y}_{2 n-j}}\right)}\left(\frac{y_{n-1}}{x_{n-1}} \Delta\right)^{\delta_{i, n}} & (n+1<j \leq 2 n-1), \\
L M^{3} \frac{x_{i} y_{1} \bar{y}_{1}}{y_{i}}\left(\frac{y_{n-1}}{x_{n-1}} \Delta\right)^{\delta_{i, n}} & (j=2 n),\end{cases}
\end{aligned}
$$

where we understand $x_{0}=\bar{x}_{0}=y_{0}=\bar{y}_{0}=1, \bar{x}_{n}=x_{n}$ and $\bar{y}_{n}=y_{n}$. 
Here we define $\overline{\mathcal{R}}(x, y)=\overline{\mathcal{R}}\left(A_{2 n-1}^{(2)}\right)(x, y)=\left(x^{\prime}, y^{\prime}\right)$ by

$$
\begin{aligned}
& x_{i}^{\prime}:=y_{i} \frac{\bar{V}_{i}}{\bar{V}_{0}}, \quad \bar{x}_{i}^{\prime}:=\bar{y}_{i} \frac{\bar{V}_{0}^{\sharp} \bar{W}_{i}}{\bar{V}_{i} \bar{W}_{1}}(1 \leq i \leq n-1), \\
& x_{n}^{\prime}=y_{n} \frac{\bar{V}_{0}^{\sharp} \bar{W}_{n-1} \bar{W}_{n}}{\bar{V}_{n-1} \bar{V}_{n} \bar{W}_{1}^{2}} \frac{1+\frac{\bar{W}_{n}}{\bar{W}_{n-1}} \mu(y)}{1+\mu(y)}, \\
& y_{i}^{\prime}:=x_{i} \frac{\bar{V}_{i}^{*} \bar{W}_{1}}{\bar{V}_{0}^{\sharp} W_{i}} \bar{y}_{i}^{\prime}:=\bar{x}_{i} \frac{\bar{V}_{0}}{\bar{V}_{i}^{*}}(1 \leq i \leq n-1), \quad y_{n}^{\prime}=x_{n} \frac{\bar{V}_{0}^{2}}{\bar{V}_{n-1}^{*} \bar{V}_{n}^{*}} \frac{1+\frac{\bar{W}_{n-1}}{\bar{W}_{n}} \mu(x)}{1+\mu(x)} .
\end{aligned}
$$

9.6. Tropical $\mathbf{R}$ for $A_{2 n}^{(2)}(n \geq 2)$. We shall describe tropical R's of type $A_{2 n}^{(2)}$. We see the following lemma for $\left\{X_{2}^{(n, L)}\right\}_{L \in \mathbb{C}^{\times}}$.

Lemma 9.6. The birational map $R\left(A_{2 n}^{(2)}\right)$ is a tropical $R$ map on the $A_{2 n}^{(2)}$-geometric crystal $\left\{X_{4}^{(n, L)}\right\}_{L}$.

The proof is the same as the one for Lemma 9.3

Let us describe the explicit form of tropical $\mathrm{R}$ on $\mathcal{B}_{L}\left(A_{2 n}^{(2)}\right)$. Set $\mathcal{R}\left(A_{2 n}^{(2)}\right):=$ $\left(\eta^{-1}, \eta^{-1}\right) \circ R\left(A_{2 n}^{(2)}\right) \circ(\eta, \eta)$ where $\eta$ is as in 6.6

Let $*: \mathcal{B}_{L}\left(A_{2 n}^{(2)}\right) \times \mathcal{B}_{M}\left(A_{2 n}^{(2)}\right) \rightarrow \mathcal{B}_{M}\left(A_{2 n}^{(2)}\right) \times \mathcal{B}_{L}\left(A_{2 n}^{(2)}\right)$ be the involution defined by

$$
\begin{aligned}
\left(\left(l_{0}, l_{1}, l_{2}, \ldots, \bar{l}_{2}, \bar{l}_{1}\right),\right. & \left.\left(m_{0}, m_{1}, m_{2}, \ldots, \bar{m}_{2}, \bar{m}_{1}\right)\right)^{*} \\
= & \left(\left(m_{0}, \bar{m}_{1}, \bar{m}_{2}, \ldots, m_{2}, m_{1}\right),\left(l_{0}, \bar{l}_{1}, \bar{l}_{2}, \ldots, l_{2}, l_{1}\right)\right)
\end{aligned}
$$

that is, $*: l_{0} \leftrightarrow m_{0}, \quad l_{i} \leftrightarrow \bar{m}_{i}, \quad \bar{l}_{i} \leftrightarrow m_{i}(1 \leq i \leq n)$.

Restricting the functions $V_{i}$ and $W_{i}$ for $A_{2 n+1}^{(2)}$ to $X_{4}^{(n, L)} \times X_{4}^{(n, M)}$, we define the rational functions $V_{i}(i=0,1, \ldots, n+1)$ and $W_{i}(i=1, \ldots, n+1)$ on $\mathcal{B}_{L}\left(A_{2 n}^{(2)}\right) \times$ $\mathcal{B}_{M}\left(A_{2 n}^{(2)}\right)\left(L, M \in \mathbb{C}^{\times}\right)$as

$$
\begin{aligned}
& W_{i}:=V_{i} V_{i}^{*}+\left(M^{2}-L^{2}\right) V_{i}^{*}+\left(L^{2}-M^{2}\right) V_{i} \quad(1 \leq i \leq n+1), \\
& V_{i}=\sum_{j=1}^{2 n}\left(\theta_{i, j}(l, m)+\theta_{i, j}^{\prime}(l, m)\right)+\sum_{j=1}^{2 n+2}\left(\eta_{i, j}(l, m)+\eta_{i, j}^{\prime}(l, m)\right),
\end{aligned}
$$

where $L^{2}=l_{n} \bar{l}_{n}\left(l_{0}^{2} \prod_{i=1}^{n-1} l_{i} \bar{l}_{i}\right)^{2}, M^{2}=m_{n} \bar{m}_{n}\left(m_{0}^{2} \prod_{i=1}^{n-1} m_{i} \bar{m}_{i}\right)^{2}, \Delta:=\frac{1+\bar{\mu}(l)}{1+\bar{\mu}(m)}$, and

$$
\begin{aligned}
& \theta_{i, j}(l, m) \\
& =\left\{\begin{array}{l}
L^{2} \prod_{k=j}^{i-1} \frac{\bar{m}_{k}}{\bar{l}_{k}} \Delta^{\delta_{i, n+1}-\delta_{j, n+1}} \quad 1 \leq j \leq i \leq n+1, \\
M^{2} \prod_{k=i}^{j-1} \frac{\bar{l}_{k}}{\bar{m}_{k}} \Delta^{-\delta_{j, n+1}} \quad 0 \leq i<j \leq n+1, \\
L M\left(\prod_{k=0}^{i-1} \frac{\bar{m}_{k}}{\bar{l}_{k}}\right)\left(\prod_{k=0}^{2 n-j} \frac{m_{k}}{l_{k}}\right) \Delta^{\delta_{i, n+1}} \frac{\bar{l}_{2 n-j+1}\left(m_{2 n-j+1}+\bar{m}_{2 n-j+1}\right)}{\left(l_{2 n-j+1}+\bar{l}_{2 n-j+1}\right) \bar{m}_{2 n-j+1}} \quad 0 \leq i \leq n+1<j .
\end{array} .\right.
\end{aligned}
$$




$$
\theta_{i, j}^{\prime}(l, m)=\left\{\begin{array}{c}
L^{2}\left(\prod_{k=0}^{i-1} \frac{\bar{m}_{k}}{\bar{l}_{k}}\right)\left(\prod_{k=0}^{j-1} \frac{m_{k}}{l_{k}}\right) \Delta^{\delta_{i, n+1}}\left(\frac{1+\mu(l)}{1+\mu(m)}\right)^{\delta_{j, n+1}} \quad j \leq n+1, \\
L M\left(\prod_{k=0}^{i-1} \frac{\bar{m}_{k}}{\bar{l}_{k}}\right)\left(\prod_{k=0}^{2 n-j} \frac{\bar{l}_{k}}{\bar{m}_{k}}\right) \Delta^{\delta_{i, n+1}} \frac{m_{2 n-j+1}\left(l_{2 n-j+1}+\bar{l}_{2 n-j+1}\right)}{l_{2 n-j+1}\left(m_{2 n-j+1}+\bar{m}_{2 n-j+1}\right)} \\
n+1<j .
\end{array}\right.
$$

$$
\eta_{i, j}(l, m)=\left\{\begin{array}{l}
L^{2}\left(\prod_{k=j}^{i-1} \frac{\bar{m}_{k}}{\bar{l}_{k}}\right)\left(\frac{\bar{m}_{j-1}}{l_{j-1}}\right) \Delta^{\delta_{i, n+1}} \mu(l)^{\delta_{j, n+1}} \quad 1 \leq j \leq i, \\
M^{2}\left(\prod_{k=i}^{j-1} \frac{\bar{l}_{k}}{\bar{m}_{k}}\right)\left(\frac{\bar{m}_{j-1}}{l_{j-1}}\right) \mu(l)^{\delta_{j, n+1}} \quad 0 \leq i<j \leq n+1, \\
L M\left(\prod_{k=0}^{i-1} \frac{\bar{m}_{k}}{\bar{l}_{k}}\right)\left(\prod_{k=0}^{n-1} \frac{m_{k}}{l_{k}}\right) \Delta^{\delta_{i, n+1}} \frac{1+\mu(m)}{1+\bar{\mu}(l)} \\
L M\left(\prod_{k=0}^{i-1} \frac{\bar{m}_{k}}{\bar{l}_{k}}\right)\left(\prod_{k=0}^{2 n-j+1} \frac{m_{k}}{l_{k}}\right) \Delta^{\delta_{i, n+1}} \frac{\frac{m_{2 n-j+2}}{\bar{m}_{2 n-j+2}}+1}{\frac{\bar{l}_{2 n-j+2}}{l_{2 n-j+2}}+1} \\
L M\left(\prod_{k=0}^{i-1} \frac{\bar{m}_{k}}{\bar{l}_{k}}\right) \Delta^{\delta_{i, n+1}} i \leq n+1, j=2 n+2 . \\
\end{array}\right.
$$

$$
\eta_{i, j}^{\prime}(l, m)=\left\{\begin{array}{c}
L^{2}\left(\prod_{k=0}^{i-1} \frac{\bar{m}_{k}}{\bar{l}_{k}}\right)\left(\prod_{k=0}^{j-1} \frac{m_{k}}{l_{k}}\right)\left(\frac{l_{j-1}}{\bar{m}_{j-1}}\right) \Delta^{\delta_{i, n+1}} \bar{\mu}(m)^{\delta_{j, n+1}} \quad 1 \leq j \leq n+1, \\
L^{2}\left(\prod_{k=0}^{i-1} \frac{\bar{m}_{k}}{\bar{l}_{k}}\right)\left(\prod_{k=0}^{n} \frac{m_{k}}{l_{k}}\right) \Delta^{\delta_{i, n+1}} \frac{1+\mu(l)}{(1+\mu(m)) \mu(m)} \quad j=n+2, \\
L M\left(\prod_{k=0}^{i-1} \frac{\bar{m}_{k}}{\bar{l}_{k}}\right)\left(\prod_{k=0}^{2 n-j+1} \frac{\bar{l}_{k}}{\bar{m}_{k}}\right) \Delta^{\delta_{i, n+1}} \frac{\left(l_{2 n-j+2}+\bar{l}_{2 n-j+2}\right) \bar{m}_{2 n-j+2}}{\left(m_{2 n-j+2}+\bar{m}_{2 n-j+2}\right) l_{2 n-j+2}} \\
n+2<j \leq 2 n+1, \\
L M\left(\prod_{k=0}^{i-1} \frac{\bar{m}_{k}}{\bar{l}_{k}}\right) \Delta^{\delta_{i, n+1}} \quad j=2 n+2,
\end{array}\right.
$$

where we understand $\bar{l}_{0}=l_{0}$ and $\bar{m}_{0}=m_{0}$.

Now, we define the tropical R $\mathcal{R}\left(A_{2 n}^{(2)}\right): \mathcal{B}_{L}\left(A_{2 n}^{(2)}\right) \times \mathcal{B}_{M}\left(A_{2 n}^{(2)}\right) \rightarrow \mathcal{B}_{M}\left(A_{2 n}^{(2)}\right) \times$ $\mathcal{B}_{L}\left(A_{2 n}^{(2)}\right)$ by

$$
\mathcal{R}\left(A_{2 n}^{(2)}\right)(l, m)=\left(l^{\prime}, m^{\prime}\right)
$$


where

$$
\begin{aligned}
& l_{0}^{\prime}=m_{0} \frac{V_{0}}{V_{1}}, \quad l_{i}^{\prime}=m_{i} \frac{V_{i} W_{i+1}}{V_{i+1} W_{i}}, \quad \bar{l}_{i}^{\prime}=\bar{m}_{i} \frac{V_{i}}{V_{i+1}} \quad(1 \leq i \leq n-1), \\
& l_{n}^{\prime}=\frac{m_{n} V_{n} W_{n+1}}{(1+\mu(m)) V_{n+1} W_{n}}\left(1+\frac{m_{n} W_{n+1}}{\bar{m}_{n} W_{n}} \bar{\mu}(m)\right), \\
& \bar{l}_{n}^{\prime}=\frac{\bar{m}_{n} V_{n}}{(1+\bar{\mu}(m)) V_{n+1}}\left(1+\frac{\bar{m}_{n} W_{n}}{m_{n} W_{n+1}} \mu(m)\right) \text {, } \\
& m_{0}^{\prime}=l_{0} \frac{V_{0}}{V_{1}^{*}}, \quad m_{i}^{\prime}=l_{i} \frac{V_{i}^{*}}{V_{i+1}^{*}}, \quad \bar{m}_{i}^{\prime}=\bar{l}_{i} \frac{V_{i}^{*} W_{i+1}^{(n)}}{V_{i+1}^{*} W_{i}^{(n)}} \quad(1 \leq i \leq n-1), \\
& m_{n}^{\prime}=\frac{l_{n} V_{n}^{*}}{(1+\mu(l)) V_{n+1}^{*}}\left(1+\frac{l_{n} W_{n}}{\bar{l}_{n} W_{n+1}} \bar{\mu}(l)\right) \text {, } \\
& \bar{m}_{n}^{\prime}=\frac{\bar{l}_{n} V_{n}^{*} W_{n+1}}{(1+\bar{\mu}(l)) V_{n+1}^{*} W_{n}}\left(1+\frac{\bar{l}_{n} W_{n+1}}{l_{n} W_{n}} \mu(l)\right) .
\end{aligned}
$$

Here note that for $\left(l^{\prime}, m^{\prime}\right)=\mathcal{R}\left(A_{2 n}^{(2)}\right)(l, m)$ we have $\left(l_{1}^{\prime} l_{2}^{\prime} \cdots l_{n-1}^{\prime} \bar{l}_{n-1}^{\prime} \cdots \bar{l}_{2}^{\prime} \bar{l}_{1}^{\prime}\right)^{2} l_{n}^{\prime} \bar{l}_{n}^{\prime}=$ $M^{2}$ and $\left(m_{1}^{\prime} m_{2}^{\prime} \cdots m_{n-1}^{\prime} \bar{m}_{n-1}^{\prime} \cdots \bar{m}_{2}^{\prime} \bar{m}_{1}^{\prime}\right)^{2} m_{n}^{\prime} \bar{m}_{n}^{\prime}=L^{2}$.

Next, we shall describe tropical $\mathrm{R}$ on $\mathcal{V}\left(A_{2 n}^{(2)}\right)_{L} \times \mathcal{V}\left(A_{2 n}^{(2)}\right)_{M}$. Let $*$ be the involution $\mathcal{V}\left(A_{2 n}^{(2)}\right)_{L} \times \mathcal{V}\left(A_{2 n}^{(2)}\right)_{M} \rightarrow \mathcal{V}\left(A_{2 n}^{(2)}\right)_{M} \times \mathcal{V}\left(A_{2 n}^{(2)}\right)_{L}$ defined by $x_{0} \leftrightarrow y_{0}$ and

$$
\begin{aligned}
x_{i} \mapsto \frac{y_{0}^{2}}{M^{2} \bar{y}_{i}}, \bar{x}_{i} \mapsto \frac{y_{0}^{2}}{M^{2} y_{i}}, x_{n} \mapsto \frac{y_{0}^{4}}{M^{2} y_{n}}, y_{i} \mapsto \frac{x_{0}^{2}}{L^{2} \bar{x}_{i}}, \\
\bar{y}_{i} \mapsto \frac{x_{0}^{2}}{L^{2} x_{i}} y_{n} \mapsto \frac{x_{0}^{4}}{L^{2} x_{n}},(i=1, \cdots, n-1) .
\end{aligned}
$$

We define the rational functions $\bar{V}_{i}(x, y)(i=0,1, \ldots, n+1)$ and $\bar{W}_{i}(x, y)(i=$ $1, \ldots, n+1)$ on $\mathcal{V}\left(A_{2 n}^{(2)}\right)_{L} \times \mathcal{V}\left(A_{2 n}^{(2)}\right)_{M}\left(L, M \in \mathbb{C}^{\times}\right)$by

$$
\begin{aligned}
& \bar{V}_{i}=\sum_{j=1}^{2 n}\left(\bar{\theta}_{i, j}(x, y)+\bar{\theta}_{i, j}^{\prime}(x, y)\right)+\sum_{j=1}^{2 n+2}\left(\bar{\eta}_{i, j}(x, y)+\bar{\eta}_{i, j}^{\prime}(x, y)\right), \\
& \bar{W}_{i}:=\bar{V}_{i} \bar{V}_{*_{i}}+\left(M^{2}-L^{2}\right) \bar{V}_{i}^{*}+\left(L^{2}-M^{2}\right) \bar{V}_{i} \quad(1 \leq i \leq n+1), \\
& \mu(x):=\frac{x_{n}}{L x_{n-1} \bar{x}_{n-1}}=\bar{\mu}(x)^{-1}, \mu(y):=\frac{y_{n}}{M y_{n-1} \bar{y}_{n-1}}=\bar{\mu}(y)^{-1}, \\
& \Delta:=\frac{1+\bar{\mu}(x)}{1+\bar{\mu}(y)}, \quad \nabla:=\frac{1+\mu(x)}{1+\mu(y)}, \quad \square:=\frac{1+\mu(y)}{1+\bar{\mu}(x)}
\end{aligned}
$$

$\bar{\theta}_{i, j}(x, y):=$

$$
\left\{\begin{array}{l}
L^{2}\left(\frac{x_{i-1}}{y_{i-1}}\right)^{1+\delta_{i, 1}}\left(\frac{y_{j-1}}{x_{j-1}}\right)^{1+\delta_{j, 1}}\left(\frac{y_{n-1}}{x_{n-1}} \Delta\right)^{\delta_{i, n+1}-\delta_{j, n+1}} \quad(1 \leq j \leq i \leq n+1), \\
M^{2} \frac{x_{i-1} y_{j-1}}{x_{j-1} y_{i-1}}\left(\frac{x_{n-1}}{y_{n-1}} \Delta^{-1}\right)^{\delta_{j, n+1}}\left(\frac{x_{0}}{y_{0}}\right)^{\delta_{i, 0}+\delta_{i, 1}-\delta_{j, 1}} \quad(0 \leq i<j \leq n+1), \\
L M \frac{x_{i-1}}{y_{i-1}}\left(\frac{M^{2} \bar{y}_{2 n-j}}{L^{2} \bar{x}_{2 n-j}}\right)^{1-\delta_{j, 2 n}} \frac{1+M^{2 \delta_{j, 2 n} \frac{y_{2 n+1-j} \bar{y}_{2 n+1-j}}{y_{2 n-j} \bar{y}_{2 n-j}}}}{1+L^{2 \delta_{j, 2 n} \frac{x_{2 n+1-j} \bar{x}_{2 n+1-j}}{x_{2 n-j} \bar{x}_{2 n-j}}}} \quad(i \leq n+1<j<2 n),
\end{array}\right.
$$




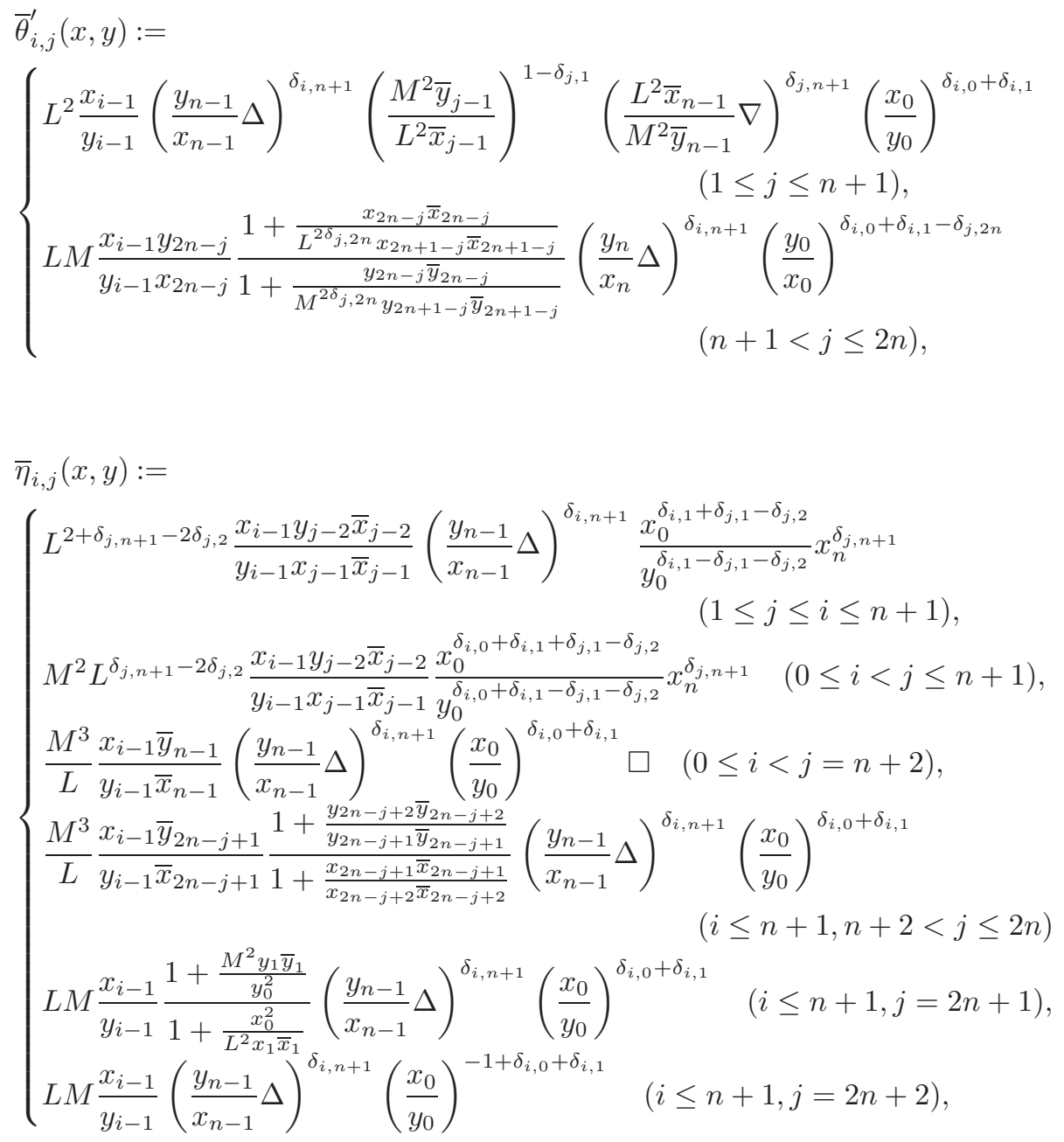

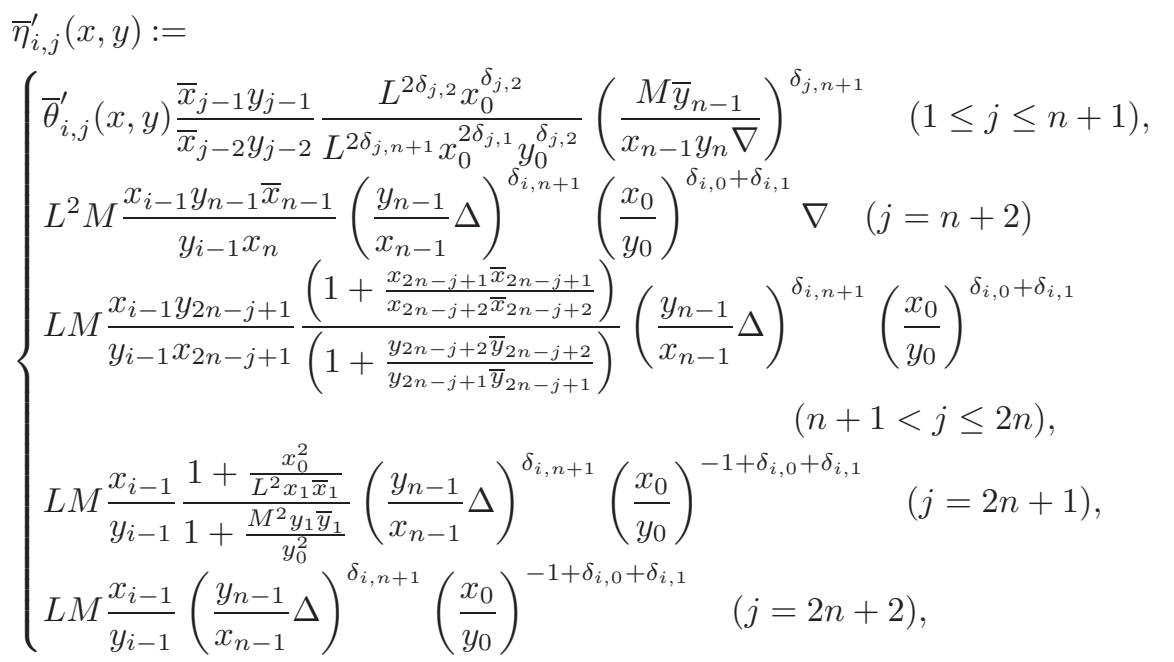


where we understand that $\bar{x}_{0}=x_{0}, \bar{y}_{0}=y_{0}$ and $x_{-1}=\bar{x}_{-1}=y_{-1}=\bar{y}_{-1}=1$. Here we define $\overline{\mathcal{R}}(x, y)=\overline{\mathcal{R}}\left(A_{2 n}^{(2)}\right)(x, y)=\left(x^{\prime}, y^{\prime}\right)$ by

$$
\begin{aligned}
& x_{0}^{\prime}:=y_{0} \frac{\bar{V}_{1}}{\bar{V}_{0}}, \quad x_{i}^{\prime}:=y_{i} \frac{\bar{V}_{1} \bar{V}_{i+1}}{\bar{V}_{0}^{2}}, \quad \bar{x}_{i}^{\prime}:=\bar{y}_{i} \frac{\bar{V}_{1} \bar{W}_{i+1}}{\bar{V}_{i+1} \bar{W}_{1}} \quad(1 \leq i \leq n-1), \\
& x_{n}^{\prime}=y_{n} \frac{\bar{V}_{1}^{2} \bar{W}_{n} \bar{W}_{n+1}}{\bar{V}_{n} \bar{V}_{n+1} \bar{W}_{1}^{2}}\left(1+\frac{\mu(y) \bar{W}_{n+1}}{\bar{W}_{n}}\right)(1+\mu(y))^{-1}, \\
& y_{0}^{\prime}:=x_{0} \frac{\bar{V}_{1}^{*}}{\bar{V}_{0}}, \quad y_{i}^{\prime}:=x_{i} \frac{\bar{V}_{1}^{*} \bar{V}_{i+1}^{*} \bar{W}_{1}}{\bar{V}_{0}^{2} W_{i+1}}, \quad \bar{y}_{i}^{\prime}:=\bar{x}_{i} \frac{\bar{V}_{1}^{*}}{\bar{V}_{i+1}^{*}}(1 \leq i \leq n-1), \\
& y_{n}^{\prime}=x_{n} \frac{\left(\bar{V}_{1}^{*}\right)^{2}}{\bar{V}_{n}^{*} \bar{V}_{n+1}^{*}}\left(1+\frac{\mu(x) \bar{W}_{n}}{\bar{W}_{n+1}}\right)(1+\mu(x))^{-1} \text {. }
\end{aligned}
$$

\subsection{Uniqueness of the tropical $\mathbf{R}$ maps.}

Theorem 9.7. Let $\overline{\mathcal{R}}$ be the tropical $R$ as introduced in this section. Set $z_{0}:=$ $\mathcal{R}(\mathbf{1}, \mathbf{1})$. Let $\mathcal{R}^{\prime}$ be a tropical $R$ such that $\mathcal{R}^{\prime}(\mathbf{1}, \mathbf{1})=\mathbf{z}_{\mathbf{0}}$. Then we have $\overline{\mathcal{R}}=\mathcal{R}^{\prime}$ as birational maps.

Proof. Let $\mathcal{V}_{l}, \mathcal{V}_{m}\left(l, m \in \mathbb{C}^{\times}\right)$be the affine geometric crystals constructed in Section 5 . By Theorem 5.4 and Theorem [7.1, we have that $\mathcal{U} D_{\left(\theta_{l}, \theta_{m}\right)}\left(\mathcal{V}_{l}(\mathfrak{g}) \times \mathcal{V}_{m}(\mathfrak{g})\right)$ is isomorphic to the crystal $B_{\infty}\left(\mathfrak{g}^{L}\right) \otimes B_{\infty}\left(\mathfrak{g}^{L}\right)$, where $\theta_{l}$ is the positive structure as in 5.10. Since $B_{\infty}\left(\mathfrak{g}^{L}\right) \otimes B_{\infty}\left(\mathfrak{g}^{L}\right)$ is connected, $\mathcal{V}_{l}(\mathfrak{g}) \times \mathcal{V}_{m}(\mathfrak{g})$ is prehomogeneous by Theorem 3.3. Therefore, by Lemma 3.2 we obtain $\overline{\mathcal{R}}=\mathcal{R}^{\prime}$, which completes the proof of Theorem 9.7 .

\section{REFERENCES}

[BK] Berenstein, A. and Kazhdan, D., Geometric crystals and unipotent crystals, GAFA 2000(Tel Aviv, 1999), Geom Funct. Anal. Special Volume, Part I, (2000), 188-236. MR:1826254 (2003b:17013)

[D1] Drinfeld, V.G., Quantum groups, Proceedings of the ICM, Berkeley, 1986. MR934283 (89f:17017)

[D2] Drinfeld, V.G., On some unsolved problems in quantum group theory, in "Quantum groups" (Leningrad 1990), Lect. Notes in Math., 1510, Springer, Berlin, 1-8, (1992). MR.1183474 (94a:17006)

[HKOTY] Hatayama, G., Kuniba, A., Okado, M., Takagi, T. and Yamada, Y., Scattering rules in soliton cellular automata associated with crystal bases, Contemp. Math. 297, 151-182 (2002). MR:1919817(2003k:81104)

[J] Jimbo, M., A $q$-difference analogue of $U(g)$ and the Yang-Baxter equation, Lett. Math. Phys. 10, 63-69 (1985). MR797001 (86k:17008)

[Kac] Kac, V.G., Infinite Dimensional Lie Algebras, Cambridge Univ. Press, 3rd edition (1990). MR 1104219 (92k:17038)

[KKM] Kang, S-J., Kashiwara, M. and Misra, K.C., Crystal bases of Verma modules for quantum affine Lie algebras, Compositio Mathematica 92 (1994), 299-345. MR1286129 (95h:17016)

[KMN1] Kang, S-J., Kashiwara, M., Misra, K.C., Miwa, T., Nakashima, T. and Nakayashiki, A., Affine crystals and vertex models, Int. J. Mod. Phys., A7 Suppl. 1A (1992), 449-485. MR.1187560 (94a:17008)

[KMN2] , Perfect crystals of quantum affine Lie algebras, Duke Math. J., 68(3), (1992) 499-607. MR 1194953 (94j:17013)

[K1] Kashiwara, M., On level-zero representation of quantized affine algebras, Duke Math. J., 112 (2002), 499-525. MR:1890649(2002m:17013) 
[K2] Level zero fundamental representations over quantized affine algebras and Demazure modules, Publ. Res. Inst. Math. Sci. 41 (2005) no. 1, 223-250. MR2115972 (2005i:17021)

[KOTUY] Kuniba, A., Misra, K.C., Okado, M., Takagi T. and Uchiyama J., Crystals for Demazure modules of classical affine Lie algebras, J. of Alg. 208 (1998) 185-215. MR 1643999 (99h:17008)

[KNO] Kashiwara, M., Nakashima, T. and Okado, M., Affine Geometric Crystals and Limit of Perfect Crystals, Trans. Amer. Math. Soc. 360 (2008), no. 7, 3645-3686. MR2386241 (2009e:17020)

[KOTY] Kuniba, A., Okado, M., Takagi, T. and Yamada, Y., Geometric crystals and tropical $\mathcal{R}$ for $D_{n}^{(1)}$, IMRN, no. 48, (2003), 2565-2620. MR2013509 (2004h:17016)

[N1] Nakashima, T., Geometric crystals on Schubert varieties, Journal of Geometry and Physics, 53 (2), (2005), 197-225. MR2110832 (2006d:17016)

[N2] _ Geometric crystals on unipotent groups and generalized Young tableaux, Journal of Algebra, 293 (2005) No. 1, 65-88. MR2173966 (2006j:20064)

[PK] Peterson D.H. and Kac V.G., Infinite flag varieties and conjugacy theorems, Proc. Nat. Acad. Sci. USA, 80 (1983), 1778-1782. MR699439 (84g:17017)

[Y] Yamada Y., A birational representation of Weyl group, combinatorial $R$-matrix and discrete Toda equation, in "Physics and Combinatorics 2000" (Eds. A.N. Kirillov and N. Liskova) Proceedings of the Nagoya 2000 International Workshop, pp. 305-319, World Scientific, 2001. MR1872262 (2002k:20021)

Research Institute for Mathematical Sciences, Kyoto University, Kitashiwakawa, SAKYO-KU, KYOTO 606, JAPAN

E-mail address: masaki@kurims.kyoto-u.ac.jp

Department of Mathematics, Sophia University, Kioicho 7-1, Chiyoda-ku, Tokyo 1028554, JAPAN

E-mail address: toshiki@mm.sophia.ac.jp

Department of Mathematical Science, Graduate School of Engineering Science, OsAKA UNIVERSITY, TOYONAKA, OSAKA 560-8531, JAPAN

E-mail address: okado@sigmath.es.osaka-u.ac.jp 